\title{
Task P:
}

\section{Experimental Particle Physics}

\author{
University of California, San Diego
}

August 20, 2013 



\section{Contents}

1 Introduction $\quad 1$

1.1 Project Goals . . . . . . . . . . . . . . . . . . . . . 1

2 Professor James G. Branson 2

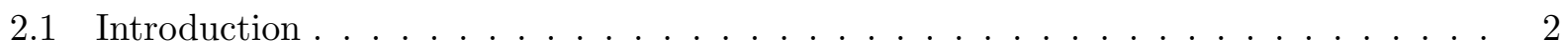

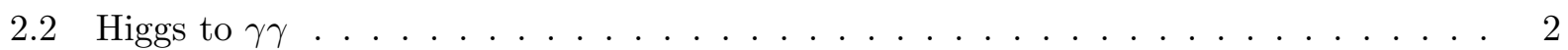

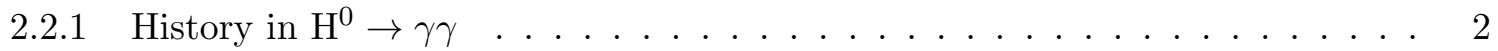

2.2.2 A Summary of UCSD Contributions to the $\mathrm{H}^{0} \rightarrow \gamma \gamma$ Analysis . . . . . . . . . 4

2.3 Data Acquisition System $(\mathrm{DAQ}) \ldots \ldots \ldots \ldots$

3 Professor Vivek Sharma $\quad 6$

3.1 Research In CMS: $2009-2012 \ldots \ldots \ldots \ldots \ldots$

3.1.1 Leadership roles in Higgs boson studies . . . . . . . . . . . . . . . 6

3.1 .2 SM Higgs boson searches . . . . . . . . . . . . . . . . . . . . . 8

3.1.3 CMS Tracker \& Charged Particle Tracking . . . . . . . . . . . . . 10

4 Profs Würthwein and Yagil $\quad 12$

4.1 CMS Physics Analysis during the last 3 years . . . . . . . . . . . . . . . . 12

4.1.1 Measurement of the top cross-section in the dilepton final state . . . . . . . 13

4.1.2 Search for Higgs in the WW to $l \nu l \nu$ final state . . . . . . . . . . . . . 13

4.1.3 Search for Higgs in the ZZ to $11 \nu \nu$ final state . . . . . . . . . . . . . . . 14

4.1.4 WW cross section measurement in the dilepton final state . . . . . . . . . 15

4.1.5 Search for heavy, top-like quark pair production in the dilepton final state . . 15

4.1.6 Search for SUSY-like signature in the opposite-sign dilepton final state outside the $\mathrm{Z}$ peak . . . . . . . . . . . . . . . . . . . 15

4.1.7 Search for SUSY-like signature in the opposite-sign dilepton final state inside the $\mathrm{Z}$ peak . . . . . . . . . . . . . . . . . . . 16

4.1.8 Search for SUSY-like signature in the Same-Sign dilepton final state . . . . . 16

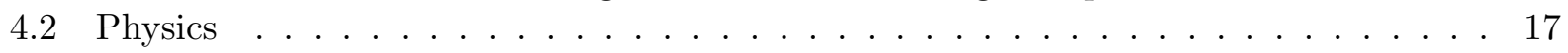

4.2.1 Prof Würthwein - Highlights . . . . . . . . . . . . . . 17

4.2.2 Prof Yagil - Highlights of Technical Contributions _ . . . . . . . . . . 18

4.2 .3 Trigger . . . . . . . . . . . . . . . . . . . . . . . 19

4.2 .4 Tracking . . . . . . . . . . . . . . . . . . . . 19

4.2.5 Fireworks: Physics Analysis Event Display . . . . . . . . . . . . . . . 19

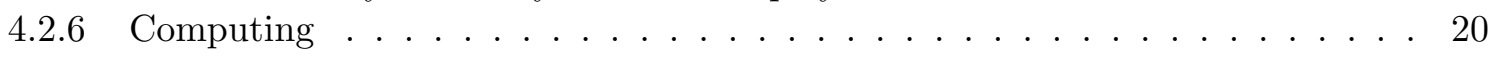

5 Bibliography and References $\quad 21$ 


\section{Chapter 1: Introduction}

\subsection{Project Goals}

The UCSD Elementary Particle Physics group investigates physics at the High Energy Frontier by working exclusively on the CMS experiment at the Large Hadron Collider at CERN. The goal of all four PIs is to discover new facets of the as-yet unwritten laws of nature as a new high-energy regime is opened at the LHC. To that end, we are primarily working on the search for the Higgs boson(s), on the search for supersymmetry, and the search for other new particles and interactions. Further, our goal is to have a very significant intellectual impact on the experiment, as we have in the past.

We have played a leading role in the Higgs search in the past and particularly in the observation of a new boson that is consistent with a Higgs-like particle. All four PIs are very involved in the Higgs search. Vivek Sharma was the CMS Higgs co-coordinator during 2010-11. One of our key goals is to measure the properties of this particle as accurately as possible during the upcoming proposal period. The most important modes for this measurement are $\mathrm{H}^{0} \rightarrow \gamma \gamma, \mathrm{H}^{0} \rightarrow \mathrm{ZZ}$, and $\mathrm{H}^{0} \rightarrow \mathrm{WW}$. We have played leading roles in the measurement of these channels up to now and our goal is to continue work in these channels, measuring the production modes of the "Higgs", the spin and parity, and the decay branching fractions.

If the particle we observe is a light Higgs, it is "natural" that there is other new physics at or below the TeV scale. The combined groups of Avi Yagil and Frank Würthwein have been highly productive in the search for supersymmetry and other new particles using primarily a search strategy based on (di-)leptons and missing transverse energy.

One of our key goals has always been to make a difference in the experiment. We have made important technical, intellectual, and organizational contributions to the experiment. Below we list some of them (roughly chronologically):

- Jim Branson and Ian Fisk took a leading role in the early USCMS computing project helping to establish it as a success and initiating one of the first Tier-2 computing centers. With Frank Würthwein taking a leading role in CMS computing, our Tier-2 center has become one of the best in CMS, particularly in terms of the breadth and depth of the physics results produced there.

- Jim Branson and Marco Pieri played a key role in the design and implementation of the CMS Data Acquisition allowing us to have a highly reliable and high-performance system that can be maintained and operated with a relatively small crew.

- Jim and his students first showed that the $\pi^{0}$ mass peak could be used to inter-calibrate the 80000 crystals of the detector. This is still the primary inter-calibration method.

- Avi Yagil lead the redesign of the CMS Event-Data-Model and the CMS software framework, implementing the new system currently in use by CMS. CMS has greatly benefitted from the upgradability and maintainability of CMSSW.

- Vivek Sharma took on the problem of starting up the CMS publications system as deputy chair of the publications committee. He took the lead in building the analysis review and publication system that works very well for us today.

- Frank and the UCSD computing group have innovated and developed the software system used to make grid-computing work so successfully in CMS.

- Marco Pieri has been in some significant leadership role for every one of the last five years. He moved from $\mathrm{H}^{0} \rightarrow \gamma \gamma$ coordinator to $\mathrm{e} / \gamma$ coordinator and is now $\mathrm{H}^{0} \rightarrow \gamma \gamma$ coordinator again since January. He was highly effecetive in these groups due to his experience.

- Avi Yagil initiated and lead the creation of a maintainable and highly useable event-display program and rather quickly produced a product that is widely used for viewing events. 
- Post docs Mangano Petrucciani (Sharma group) and Cerati (Yagil group) have been key contributors to the implementation, development and speed-up in the presence of very high pileu of the CMS tracking code. Managano was tracking co-coordinator for 2 years and Cerati will be tracking co-coordinator for 2013-14.

- Jim and Frank helped to understand the one big detector problem that CMS had at startup - anomalous high-energy hits in the calorimeter causing fake missing ET and other problems.

- As the CMS Higgs search coordinator, Sharma rebuilt the Higgs group and led it towards rapidly producing a comprehensive set of very high quality results for conferences and publications. He also played a leadership role in the formation and activities of the LHC Higgs combination group.

- Postdoc Petrucciani is the co-convener of the CMS "Higgs combination and properties" group for 2012-13. He is the chief architect of the statistical procedures and framework used to combine the results of various Higgs searches and measurement of its properties.

Many of the contributions listed above have made CMS a more efficient experiment. We have been able to process and reprocess our data quickly due to the DAQ-software-computing system that UCSD helped build. CMS has been able to promptly produce a wide range of physics results.

\section{Chapter 2: Professor James G. Branson}

\subsection{Introduction}

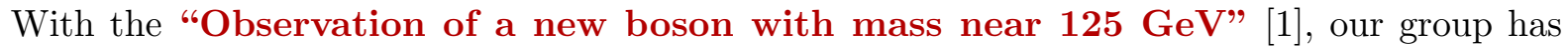
reached a milestone in the $\mathrm{H}^{0} \rightarrow \gamma \gamma$ analysis in which we have played a leading role since early in CMS. We have made fundamental contributions both to this analysis and to the triggering, reconstruction, identification, and measurement of photons and electrons. We have also worked on the CMS Data Acquisition system (DAQ) since the USCMS collaboration was formed in 1994. The UCSD group made very important contributions to the design, construction, and operation of the highly successful CMS DAQ.

\subsection{Higgs to $\gamma \gamma$}

2.2.1 History in $\mathrm{H}^{0} \rightarrow \gamma \gamma$ : Our group pioneered the $\mathrm{H}^{0} \rightarrow \gamma \gamma$ analysis in CMS. Jim Branson and Marco Pieri provided the analysis for the CMS Physics TDR showing that the SM Higgs could be discovered with much less luminosity than previously thought. Our analysis [2] was put right at the front of the Physics TDR [3] and now, the $\mathrm{H}^{0} \rightarrow \gamma \gamma$ analysis is the most significant one in which CMS has observed the new boson near $125.3 \mathrm{GeV}$.

We have always felt that the SM Higgs would be at low mass if it exists and that $\mathrm{H}^{0} \rightarrow \gamma \gamma$ would be an important channel for the discovery. Further, we felt that it is a very challenging channel to analyze and that improvements in the $\mathrm{H}^{0} \rightarrow \gamma \gamma$ analysis would make an important contribution due to the relatively large amount of luminosity needed for discovery at low mass.

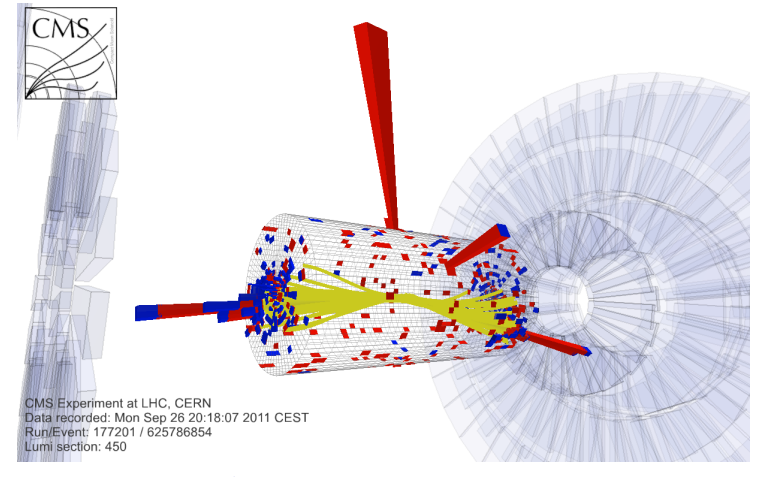

Figure 2.1: A 2011 event containing a high PT diphoton tagged a high mass dijet.

Also in the time period before the Physics TDR, the increasing estimate of the material in the tracker was making the then baseline calibration plan, using electrons from $\mathrm{W}$, unrealistic. It would take more time to intercalibrate the detector than to discover the low mass Higgs because 
electron resolution was deteriorating. The UCSD group first demonstrated that a clean $\pi^{0}$ mass peak could be reconstructed in the ECAL. Since this peak was dominated by unconverted photons the resolution was quite good. We then made a major effort to show that the ECAL could be intercalibrated in a short time using the $\pi^{0}$ peak. Some of these results appear in the Physics TDR [4]. Our method was adopted by the ECAL group and has been the primary method for intercalibration throughout all CMS runs [5]. Needless to say, ECAL intercalibration to the sub percent level is vital for $\mathrm{H}^{0} \rightarrow \gamma \gamma$.

After the Physics TDR, we continued activity in the $\mathrm{H}^{0} \rightarrow \gamma \gamma$ analysis which became more difficult due to increased tracker material causing the mass resolution in the best category to increase to $1 \%$ compared to $0.6 \%$ in the TDR, lower LHC energy, and more pileup. We spent significant time on the DAQ and on the reconstruction and identification algorithms for electrons and photons. Marco Pieri was coordinator of the $\mathrm{H}^{0} \rightarrow \gamma \gamma$ analysis subgroup in 2009. He then became coordinator of the e-gamma group, which is in charge of Electron and Photon algorithms during the crucial startup runs in 2010 and 2011, when we needed to get the reconstruction and ID working well.

Our group played a key role in the development of a multivariate analysis for use in the inclusive $\mathrm{H}^{0} \rightarrow \gamma \gamma$ analysis. We had used a neural net at the time of the physics TDR to discriminate between high $s / b$ events and low. We proposed the more conservative idea of separating events into categories based on a multivariate analysis (MVA)for 2011, so that the low $s / b$ events and the events with poor mass resolution did not pollute the mass distribution from high $s / b$ events. The low $s / b$ events, considered separately still contribute to the performance of the analysis. We also pushed the idea that the photon ID for each photon was a key component of such a multivariate technique to estimate $s / b$. We showed the first full use of all the variables including photon ID which gave a very large improvement in the analysis, separating the events into categories. One of the key improvements was a very good event-by-event estimate of the mass resolution developed by others in the $\mathrm{H}^{0} \rightarrow \gamma \gamma$ group in conjunction with the ECAL calibration group.

The final MVA gave the same performance as did ours. At that time, we spent a lot of our resources getting the

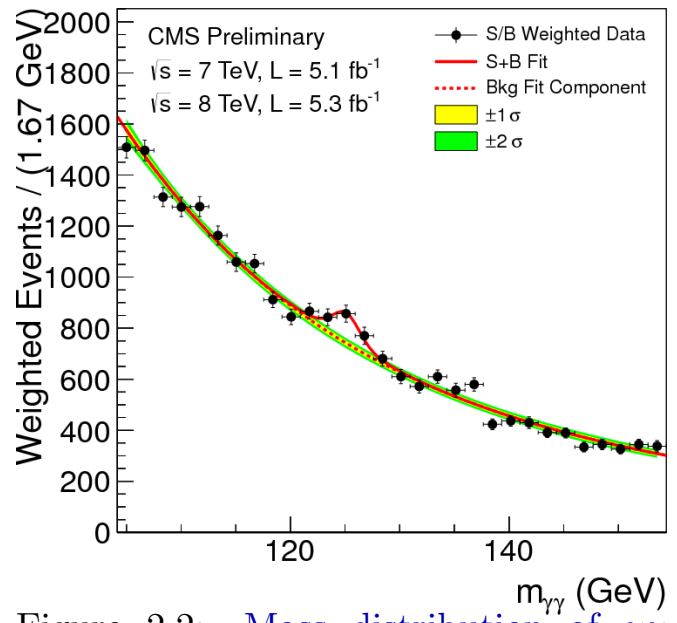

Figure 2.2: Mass distribution of $\gamma \gamma$ events. One weight per category is calculated as the expected $\frac{s}{s+b}$ near the peak, is to reflect how the fit in categories works. The weights are normalized to preserve the number of signal events, $\approx 160$ in the observed peak. VBF analysis working and approved. While we did not propose to use our MVA, we did help drive the design of the MVA method and provide a check and an alternate implementation.

For Moriond EW, the most important winter conference, the $\mathrm{H}^{0} \rightarrow \gamma \gamma$ group made an update of the $\mathrm{H}^{0} \rightarrow \gamma \gamma$ analysis using the MVA categorization [12, 13]. The MVA includes diphoton kinematics, mass resolution, photon ID, and vertex choice information to deal with pileup. We searched for a mass peak in each of the categories. The VBF channel has been left basically unchanged compared to the publication with the only difference being the usage of the MVA based photon ID. The CMS talk on the Higgs search was given by Marco Pieri [14]. Comparing with the competitor experiment, ATLAS, we had a more sophisticated analysis due to the VBF channel and the use of the MVA to categorize. Overall, we had a sensitivity approximately $20 \%$ better in cross section. To get a similar improvement from more running, one would need to increase integrated luminosity by more than $50 \%$. 
Marco Pieri was selected as the new $\mathrm{H}^{0} \rightarrow \gamma \gamma$ convener for 2012 . He is expected to serve for two years covering the discovery phase of the the "Higgs-like" boson as well as the measurements using data up to the end of 2012. During the hectic analysis and presentation of results around ICHEP, Marco has played a key role in the $\mathrm{H}^{0} \rightarrow \gamma \gamma$ group.

By the time of the Moriond result, the CMS H $\mathrm{H}^{0} \rightarrow \gamma \gamma$ analysis was well optimized for discovery of the Higgs. This analysis was changed very little for the results presented in 2012. We have stayed a step ahead of the competing experiment ATLAS for each of the presentations of the 2011 results, with ATLAS often adopting our methods by the time of the next presentation. We maintained blindness to any signal around $125 \mathrm{GeV}$ up to just before the ICHEP conference. We also made only very minor changes in the analysis. One change that was made was to split the dijet tagged category (for VBF) into two categories using the dijet mass. Since the reconstruction program was updated, all MVAs and corrections had to be recomputed. Of course calibrations and energy scale had to be redone using the 2012 data.

We were also among the main proponents of a search for a Fermiophobic Higgs where $\mathrm{H}^{0} \rightarrow \gamma \gamma$ plays a key role. As previously mentioned we carried out the exclusive analysis of the main production modes and we obtained a result that was made public for the 2012 winter conferences. By combining the results from $\mathrm{H}^{0} \rightarrow \gamma \gamma$ with the $\mathrm{H} \rightarrow \mathrm{WW}$ and $\mathrm{H} \rightarrow \mathrm{ZZ}$ analyses, we were able to exclude at $95 \%$ CL the existence of a Fermiophobic Higgs boson with mass between 110 and $190 \mathrm{GeV}[16,15,17]$.

2.2.2 A Summary of UCSD Contributions to the $\mathrm{H}^{0} \rightarrow \gamma \gamma$ Analysis: Many of the important ideas of the $\mathrm{H}^{0} \rightarrow \gamma \gamma$ analysis were already present in the CMS Physics TDR analysis done by Jim Branson and Marco Pieri. These include:

- The division into categories so that good-resolution, high $s / b$ events are not diluted with $\gamma \gamma$ events with worse resolution and more background. At the same time the somewhat worse event, can still contribute significantly to the result. We divided using physically motivated categories, based on whether the photon was measured to convert early and on the pseudorapidity of the photons. The division we used made about a $25 \%$ improvement in the cross section limit, and went most of the way toward what we have now, which is division using an MVA.

- An MVA (neural net) to do the photon ID. Of course with real data we started with a cut-based analysis and studied the variables in the data before proceeding to an MVA.

- A diphoton MVA (neural net) to help separate high $s / b$ diphotons from low using kinematics and photon ID. This MVA was used in the physical categories separately so that resolution was partially taken care of.

- A fit to signal plus background with significant flexibility in the background model. The signal model was derived from MC.

- The MVA based analysis was compared to a pure cut based analysis. Systematic errors were studied to some extent but not very well for the MVA analysis at the time of the TDR.

- An algorithm to choose the best vertex based on particles recoiling against the diphoton was used, however, the TDR assumed low luminosity and 25 nsec bunch spacing so the number of pileup events was small.

This signal coincided with that from the $\mathrm{H}^{0} \rightarrow \gamma \gamma$ analysis of the 2011 data, which we had decided to leave unchanged for the ICHEP conference result, part of our plan to have a really unbiased look at the $125 \mathrm{GeV}$ region for ICHEP. In both the $7 \mathrm{TeV}$ run of 2011 and the $8 \mathrm{TeV}$ run of 2012, the signal strength found was in excess of what was expected in our model of the Standard Model Higgs. Our expectation was that the $\mathrm{H}^{0} \rightarrow \gamma \gamma$ analysis would be the dominant channel for Higgs masses of $120 \mathrm{GeV}$ or less but that other channels would be as powerful for masses around $125 \mathrm{GeV}$, however, the $\mathrm{H}^{0} \rightarrow \gamma \gamma$ analysis is really the cleanest discovery channel because of 
the narrow mass peak. The $\mathrm{ZZ}$ channel is also very clean but suffers from low statistics and is easily affected by analysis choices when individual events come or go. The expected performance of the $\gamma \gamma$ analysis was only a little better than that of ATLAS which had again updated toward our analysis techniques, whereas we kept a stable analysis in light of the potential discovery.

We knew quickly that there was more than a 4 sigma effect in the combined $7 \mathrm{TeV}$ and 8 $\mathrm{TeV}$ data in $\mathrm{H}^{0} \rightarrow \gamma \gamma$, and that we had done the analysis in a very careful and unbiased way so that the signal was not manufactured or artificially enhanced by the analysis. When the result of the analysis in 6 categories (plus 5 for 2011) was shown to the collaboration, we experienced the same problem that we had seen in the Physics TDR. That is, people could not get a visual impression that was consistent with 4.1 sigma. Jim Branson had developed a way of combining the mass distri-
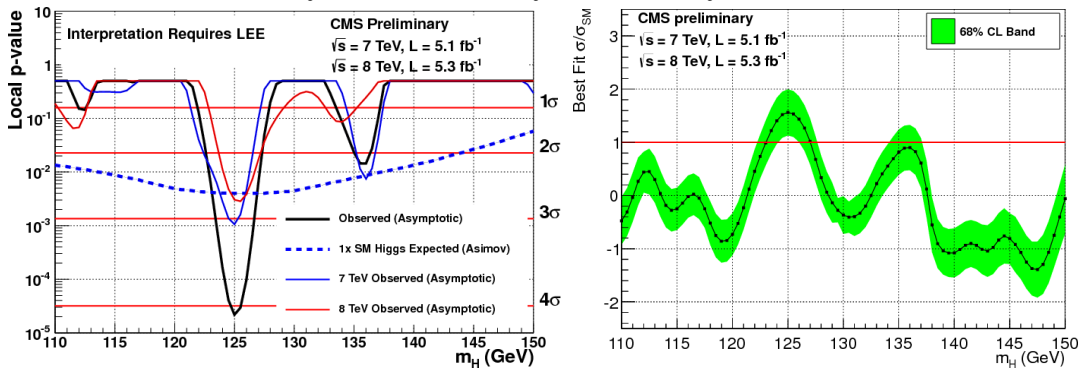

Figure 2.3: P-value (left) and best-fit- $\sigma \times B R$ in units of the SM (right), as a function of mass. butions from all the categories into one mass plot mimicking how the maximum likelihood fit effectively weighted each category to derive a significance. That is one weight per category was computed, equal to the $\frac{s}{s+b}$ expected in a small region around the peak. This includes both the signal to background in the whole mass plot and the resolution expected in that category for the signal. Since the TDR analysis we knew that good resolution was highly correlated with lower background. When the $\mathrm{H}^{0} \rightarrow \gamma \gamma$ group produced this plot, shown in Figure 2.2, the nature of the excess at 125 appeared clearly. After some initial criticism from ATLAS, finally they also included a weighted mass plot in their publication. It may be interesting to note that just such a mass plot based solely on Monte Carlo (and looking very similar) appeared in our 2009 proposal to DOE.

The size of the effect at $125 \mathrm{GeV}$ is quantified in Figure 2.3 which gives the p-value (probability to get data from background only) as a function of mass. The size of the signal observed is about 1.6 times larger than expected in the SM. It will be very interesting to see if this trend continues with more data. We also exclude [20,21] a Fermiophobic Higgs at the 99\% CL, in the mass range of 110 to $147 \mathrm{GeV}$.

\subsection{Data Acquisition System (DAQ)}

One of the key differences between CMS and ATLAS is in the DAQ system. While ATLAS has the traditional Level-1 and Level-2 trigger systems underground, CMS sends a much larger event rate (fifty times ATLAS) to a large farm of standard processors on the surface, allowing processing with highly flexible software derived from the offline analysis. This means CMS can rapidly change the High Level Trigger (HLT) that is specified to reduce from a maximum Level-1 rate of $100 \mathrm{kHz}$ to a few hundred $\mathrm{Hz}$ to be sent to mass storage.

The UCSD group proposed and first tested an implementation of the Event Builder in Gigabit Ethernet, first with custom protocols but ultimately using TCP/IP. Our design was one of two fully tested by CMS before the final choice of technology. The UCSD group did the implementation and testing of the GbE solution at CERN and our design was finally chosen for the Event Builder technology. Subsequently we have taken the lead in the detailed design, implementation and commissioning of the Event Builder. We have important responsibilities in the areas of the Event Builder and Filter Farm.

The DAQ transfers up to 2 Terabits per second from the Front End Drivers (FEDs) to a farm of standard computers which execute the HLT on each event. In the process of transfer, 
events are built. The capability of sending $100 \mathrm{kHz}$ of data and then analyzing full events allows us to efficiently select the most interesting events as well as to process high rate calibrations or special physics channels online. Two physics examples are the first observation of two-particle long-range correlations in pp collisions[35] and the measurement of $\mathrm{B} \rightarrow \mu \mu[36,37]$.

The system was fully ready in 2009. The efficiency of the DAQ system, from the readout at the detector Front Ends to the storage of data on disk, was 99.7\% in 2011. Filter Farm PCs have been added before both the 2011 and 2012 runs to cope with the larger pileup and to allow for more complex HLT algorithms. The CPU time available to the HLT per event was initially about $50 \mathrm{~ms}$, and is now about $165 \mathrm{~ms}$. For both upgrades, the expansion was coordinated by the UCSD group. We participated in the selection of PCs and took care of the procurement of the network equipment and of the reorganization of the GbE network and of the interconnections between the PCs and the switches. We carried out the installation of the cables the reconfiguration of the network and the recabling that was needed at the switch end.

During the last three years we made invaluable contributions to the operation of the DAQ. We are in charge of the Myrinet configuration (routing), of the application software which uses the data network (ATCP), and of the Gigabit Ethernet network configuration and monitoring for which we are on-call all the time and we take care of the necessary replacement of the failing equipment. We also maintain the general monitoring program of the CMS data acquisition.

During the data taking periods, we also provide about $20 \%$ of the on-call DAQ expert service for CMS. On-call DAQ experts are responsible for the setup of the central DAQ data taking, they participate in daily run meetings, and take day-to-day decisions concerning DAQ operations. This is a demanding task because one of the DAQ experts is on call $24 / 7$ for almost 365 days per year to quickly solve all problems that go beyond simple DAQ operation. DAQ work is done partly with support from the USCMS M\&O but mainly with base program people supported to be at CERN by the project. Vital DAQ expertise is supported by the base program.

Sergio Cittolin who guided the CMS DAQ since the beginning and was trigger and DAQ project manager until 2008 has been part of the UCSD group since 2010 . He continues to maintain the global DAQ monitoring program that he personally developed and that is of paramount importance for the monitoring of the full CMS detector operation.

In 2011 and 2012 Andre Holzner measured the data rate as a function of the number of pileup vertices for each front-end of the detector readout, due to rate limitations there. Some corrective actions in detector readouts had to be taken but the problems were found and corrected before any dead time was incurred.

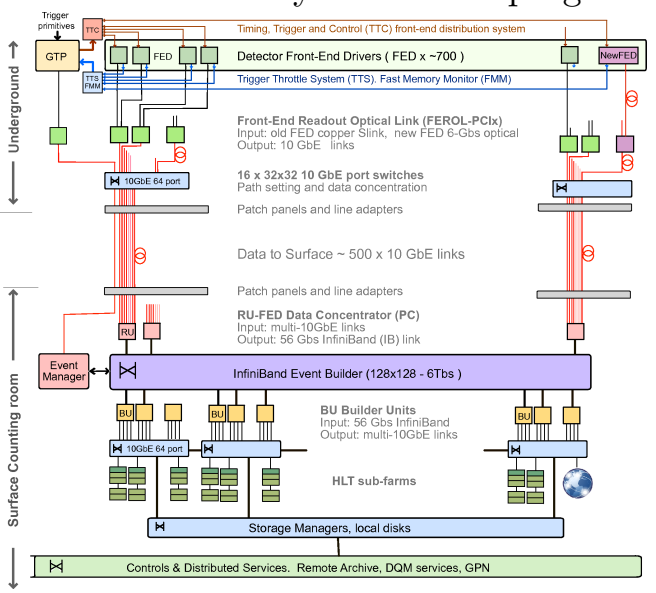

Figure 2.4: Sketch of the upgraded CMS DAQ system.

\section{Chapter 3: Professor Vivek Sharma}

\subsection{Research In CMS: 2009-2012}

\subsubsection{Leadership roles in Higgs boson studies:}

Higgs co-convener: Sharma was appointed as the CMS Higgs group co-convener in January 2010. At that time the thinking (based on 2005 physics technical design report) was that Higgs searches would be a marathon requiring a large integrated luminosity not expected during $7 \mathrm{TeV}$ 
running of the LHC. Consequently the CMS Higgs working group had shrunk to just a handful of physicists with majority of CMS focussing on SM measurements and searches for BSM physics. Since the physics TDR, CMS had made major innovations and improvements in several aspects of event reconstruction whose net impact on Higgs search sensitivity was not studied. By ICHEP'10, it became very clear that both LHC and CMS were off to a terrific start with a very fast commissioning phase. Almost every aspect of CMS reconstruction worked flawlessly "out of the box" and as per expectations from detector simulation. With LHC projecting to deliver in excess of $1 \mathrm{fb}^{-1}$ in 2011, the need for re-evaluating CMS sensitivity to Higgs boson searches became paramount. The challenges were to (a) quickly reconstitute the Higgs group, (b) expand the menu of SM and BSM Higgs search modes well beyond those considered in the PTDR (c) conduct feasibility studies using state-of-the-art CMS object performance, (d) formulate a comprehensive Higgs search strategy and (e) demonstrate that CMS had an overall sensitivity, at $95 \% \mathrm{CL}$, to rule out a large range of possible Higgs boson masses. The assessment of CMS capability with $1 \mathrm{fb}^{-1}$ computed by Korytov \& Sharma first at Sept'10 CMS Physics week came as a surprise and was critical in re-energizing the CMS collaboration's focus towards Higgs searches. CMS management quickly classified Higgs searches as the collaboration's highest priority activity in 2011. These projections (CMS-NOTE 2008/008), along with independent but similar estimates from ATLAS, became the key input to the LHCC towards the LHC run plans for 2011-12. Sharma organized a two-day internal Higgs review in Dec'10 to substantially grow the manpower in the Higgs group and define the path towards preparation for 2011 data. These efforts started bearing results rather quickly. Four competitive searches $\left(\mathrm{H} \rightarrow \mathrm{WW}^{(*)} \rightarrow 2 \mathrm{l} 2 \nu, \mathrm{H} \rightarrow \tau \tau, \mathrm{H}^{++} \rightarrow \ell^{+} \ell^{+}\right.$and $\left.\mathrm{H}^{-} \rightarrow \tau \nu\right)$ based on $36 \mathrm{pb}^{-1}$ data accumulated in 2010 were published by spring 2011. By summer'11, the Higgs group had grown to more than 400 active physicists covering all major search channels. At EPS'11, results from $1 \mathrm{fb}^{-1}$ data accumulated at $7 \mathrm{TeV}$ in eight Higgs decay channels and their combination were presented and were in excellent agreement with the sensitivity projected in Sept'10 asessment. For the LP'11 conference just a month later, all results were updated with additional data accumulated in July. The rapid transformation of the Higgs group's size \& productivity in a period of less than a year is considered one of the success stories in CMS. Just six weeks after accumulating the full 2011 dataset, CMS presented its entire suite of SM Higgs search results at the CERN seminar on 13th Dec'11. SM-like Higgs boson was excluded at 95\% CL in the 127-600 GeV mass range and a excess with a local significance of $2.6 \sigma$ at $M_{H} \approx 124 \mathrm{GeV}$ was reported. In Oct'11, Sharma ( \& Paus) developed an aggressive timeline towards the publication of all SM Higgs searches and their combination. The collaboration-wide review of these nine papers started during the Xmas holidays. By March'12, all nine SM Higgs search publications were submitted for publication far ahead of ATLAS. With the additional $5 \mathrm{fb}^{-1}$ data accumulated at $8 \mathrm{TeV}$, and with analyses little changed from 2011, CMS reported on the observation $(4.9 \sigma)$ of a new SM Higgs-like boson with a mass near $125 \mathrm{GeV}$.

Higgs Combination \& Properties group co-convener: The combination of all Higgs search channels to achieve maximal sensitivity to characterize a possible signal has been an important task in the CMS Higgs program since 2010. Postdoc Petrucciani has been the principal architect of the statistical framework for combining the results of various Higgs searches. From the beginning he has served as the main "go to" person in the technology transfer of the combination tools to dozens of CMS physicists working on individual search channels. He anchored all Higgs combination activities and was instrumental in producing results shown at major conferences (EPS'11, LP'11, Moriond'12, ICHEP'12) and for CMS publications. In Spring'12, Giovanni was appointed as the co-convener of the "Higgs combination \& properties" subgroup for two years.

Higgs editorial board \& Analysis review committees: In Jan'12, the CMS publications board was reorganized along physics themes. Sharma, along with M. Della Negra, T. Virdee, J. 
Konigsberg, E.Gallo, and E.Longo were appointed to the Higgs editorial board. Its immediate task was to review and finalize the nine publications on SM Higgs searches based on 2011 data. To date, the Higgs editorial board has overseen the successful publication of 16 Higgs search papers. A steady stream of publications are planned over the next years.

Analysis review committees (ARC) consisting of 3-4 physicists are charged by the CMS collaboration to review analyses forwarded by the CMS physics groups and guide them towards a conf. paper or a journal publication. Sharma has served on more than 24 ARCs and chaired more than a dozen of them.

\subsubsection{SM Higgs boson searches:}

Search for $\mathrm{H} \rightarrow \mathrm{WW}^{(*)} \rightarrow 2 \mathrm{l} 2 \nu$ : This analysis searches for an excess of events with two leptons of opposite charge, large $\mathrm{E}_{\mathrm{T}}^{\mathrm{miss}}$ and up to two jets. Due to large decay branching ratios, this mode has high sensitivity but because of missing neutrinos, the Higgs mass resolution is poor $(\approx 20 \mathrm{GeV})$. Major sources of background stem from non-resonant WW, DrellYan, $\mathrm{W}+$ jets, $\mathrm{W} \gamma$ and top production. To optimize the search sensitivity, events are divided into six categories each with different background composition and signal-to-background ratios. Events with no identified jets form the most sensitive search category. Due to the complexity of this search, two teams were formed which developed various elements of the analysis independently, cross-checked each other and finally merged them into a single CMS result. Postdocs Mangano and Petrucciani along with students Lebourgeois \& Vartak collaborated with physicists from ETH, Rome, Caltech \& Oviedo and produced one of the two end-to-end analysis with cut-based and shape-based methods. Our work is documented in CMS analysis notes AN-11-201, 364, 460 $\& 463$. Mangano was the co-editor of the notes AN-11-148 $\& 432$ which served as the support technical document for the conference summaries (HIG-11-003 (EPS'11), HIG11-014 (LP'11) ) and the publication (Phys. Lett. B 710 (2012)) on the $7 \mathrm{TeV}$ data. SM Higgs boson was excluded (Figure 3.1) in the 132-238 (129-270) GeV range with the cut-based (shape-based) method. The contributions of our group to this analysis included: (a) a novel algorithm for primary vertex reconstruction robust against high pile-up conditions (b) algorithm to identify \& reject decay-in-flight muons in $\mathrm{W}+$ jet events (c) Re-tuning of lepton identification to improve low mass Higgs search ef-

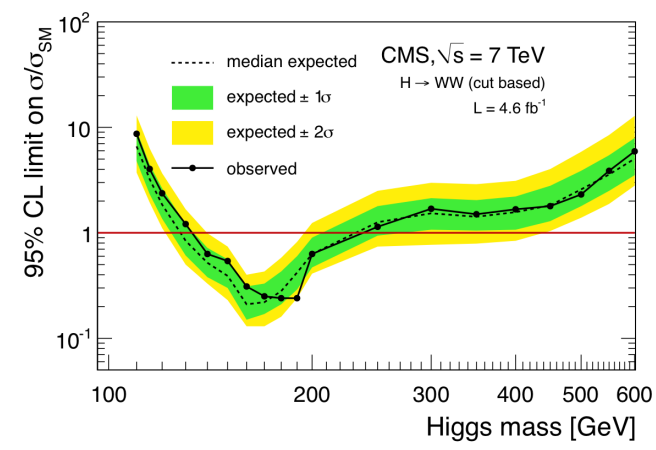

Figure 3.1: $95 \%$ CL limit on $\sigma_{H} \times$ $\operatorname{Br}(H \rightarrow W W)$ from a cut-based analysis of the $5 \mathrm{fb}^{-1}$ data at $7 \mathrm{TeV}$. ficiency (d) Data-driven method to estimate W+jets background (e) Implementation of theoretical uncertainties in 0,1,2 jet categorization (f) Implementation of a shape based analysis using single observables such as dilepton invariant mass and (g) studies of shape uncertainties in the multivariate analysis.

Search for $\mathrm{H} \rightarrow \mathrm{ZZ}^{(*)} \rightarrow 2 \mathrm{l} 2 \nu$ : Before 2010, the CMS effort on the $\mathrm{H} \rightarrow \mathrm{ZZ}$ mode was limited to $\mathrm{H} \rightarrow \mathrm{ZZ}^{(*)} \rightarrow 41$. Prior studies had concluded that all other ZZ channels were hopeless due to large backgrounds. With the successful commissioning of sophisticated new tools for Jet, MET and b-tagging, Vartak (\& Sharma) initiated a set of feasibility studies in summer'10 which demonstrated that $\mathrm{H} \rightarrow \mathrm{ZZ}^{(*)} \rightarrow 2 \mathrm{l} 2 \nu, \mathrm{H} \rightarrow \mathrm{ZZ}^{(*)} \rightarrow 2 \mathrm{l} 2 \mathrm{q}$ and $\mathrm{H} \rightarrow \mathrm{ZZ}^{(*)} \rightarrow 2 \mathrm{l} 2 \mathrm{~b}$ modes have, in fact, good sensitivity in high mass $(\mathrm{M} \geq 250 \mathrm{GeV})$ Higgs searches. Vartak then assisted several new groups joining Higgs search in contributing to these modes. Since the beginning, Vartak led all aspects of the $\mathrm{H} \rightarrow \mathrm{ZZ}^{(*)} \rightarrow 2 \mathrm{l} 2 \nu$ analysis. With colleagues from Florida and Purdue, he developed an end-to-end analysis including techniques for background suppression, data 
driven methods to estimate residual contamination from WW, Top and $\mathrm{Z}+$ jets background and documented it in CMS note AN-11-453. He was the co-editor (with Drozdetskiy, UF) of the conferences notes (HIG-11-005, HIG-11-016), the supporting technical documents (AN-119, AN-407) and the publication based on full 2011 data ( JHEP, 03 (2012)) which ruled out a SM Higgs in the mass range $270<M_{H}<440 \mathrm{GeV}$ at $95 \%$ CL. With our group's focus shifting to $\mathrm{H} \rightarrow \mathrm{ZZ}^{(*)} \rightarrow 4 \mathrm{l}$ channel in 2012, the Purdue and CERN group took over the update of this analysis for the ICHEP' 12 conference (CMS PAS HIG12-023). With the additional $5 \mathrm{fb}^{-1}$ data at $8 \mathrm{TeV}$, a SM Higgs boson was excluded (Figure 3.2 ) in the 278-600 GeV mass range from this channel alone. Sharma is the chair of the review committee for this analysis.

Search for $\mathrm{H} \rightarrow \mathrm{ZZ}^{(*)} \rightarrow 4 \mathrm{l}:$ With the exclusion of a SM Higgs boson in the 127-600 GeV mass range with the $7 \mathrm{TeV}$ data, our focus in 2012 turned to the investigation of the excess around $125 \mathrm{GeV}$ with the $7 \& 8 \mathrm{TeV}$ data using the $\mathrm{H} \rightarrow \mathrm{ZZ}^{(*)} \rightarrow 4 \mathrm{l}$ channel. This "golden " channel has an excellent mass resolution (1-2 \%) and much smaller background compared with the $\mathrm{H} \rightarrow \gamma \gamma$ mode but also smaller number of reconstructed Higgs signal events. Our group has focussed on
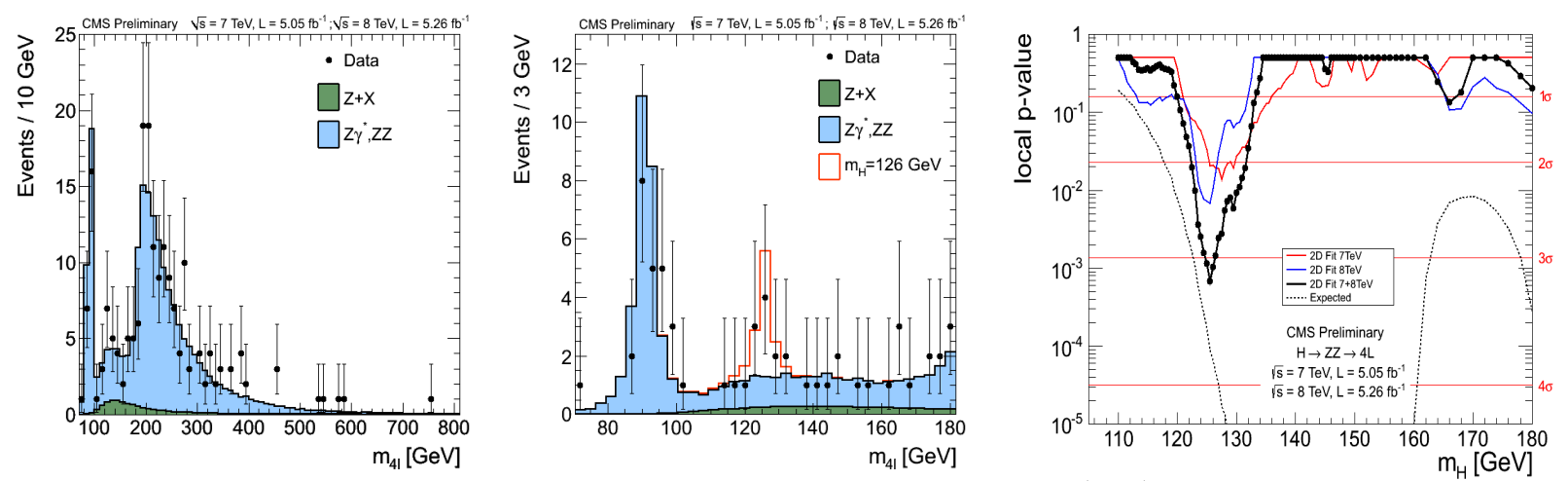

Figure 3.3: Distribution of the $4 \ell$ mass in the range of 70-800 GeV (left), zoom around 70-180 GeV (center) and the significance of the excess w.r.t. background expectations (right).

several aspects of the $\mathrm{H} \rightarrow \mathrm{ZZ}^{(*)} \rightarrow 4 \mathrm{l}$ analysis that led to an overall gain in discovery sensitivity by $20 \%$. Together with some Caltech colleagues, we produced an independent end-to-end analysis and documented it in CMS note AN-12-212. Some of our key contributions to this analysis were in developing algorithms for low Pt lepton identification and fake rate measurements, strategy to remove "ghost" muons candidates, implementation of tag \& probe techniques to measure trigger and identification efficiencies, independent implementation of algorithm for recovery of final state photons, measurement of reducible background (from top, Z+bb, WZ, QCD etc) and in constructing the statistical procedure to evaluate limits and local p-values in the $2 \mathrm{D}$ distribution of $M_{4 l}$ Vs. a discriminant based on the $Z$ masses \& the angular information in the $Z Z \rightarrow 4 l$ decay. The key results from the analysis of $10 \mathrm{fb}^{-1}$ data accumulated by June'12 is shown in Figure 3.3. A cluster of events with a local significance of $3.2 \sigma$ over expected background is observed around $M_{4 \ell}=125$ $\mathrm{GeV}$. A paper entitled "Observation of a new boson at a mass of $125 \mathrm{GeV}$ with the CMS experiment at the LHC" incorporating this result has been accepted for publication in Phy. Lett. (B).

Combination of Higgs boson searches \& properties: The combination of the CMS Higgs boson searches requires simultaneous analysis of the data selected by all individual analysis (totaling more than 95 sub-channels) accounting for all statistical and systematic uncertainties. Combining 
ATLAS \& CMS results adds yet another important layer of complexity. At the initiative of the ATLAS \& CMS spokespersons, the LHC Higgs combination group (LHC-HCG), with Sharma (CMS) and W. Murray (ATLAS) as co-conveners, was formed in Dec'10 to formulate a common statistical procedure and develop a computational framework to combine the searches. Petrucciani and Sharma along with A.Korytov and M. Chen (Florida) were the principal CMS contributors to LHC-HCG. In summer'11 ATLAS \& CMS collaborations approved a historic joint document titled "Procedure for the LHC Higgs boson Combination in Summer 2011" which defined the recommended procedures for characterizing exclusion of a signal or observation of an excess in Higgs boson searches. This document (CMS NOTE-2011-005, ATL-PHYS-PUB-2011-11) serves as the foundation of all ATLAS, CMS and ATLAS+CMS Higgs search combinations to this day. Sharma \& Petrucciani were part of the core team of about eight physicists from ATLAS \& CMS who produced and documented the LHC-HCG combination of Summer'11 Higgs search results. The joint document titled " Combined standard model Higgs boson searches with up to $2.3 \mathrm{fb}^{-1}$ of pp collision data at $\sqrt{s}=7 \mathrm{TeV}$ " was independently reviewed by both collaborations and released as a conference note (CMS PAS HIG-11-023) for the HCP conference in Nov'11.

Postdoc Petrucciani developed the ROOT-based statistical framework which incorporates the combination procedure defined by the LHC-HCG and is used in all CMS Higgs searches. This framework supports various forms of statistical modeling of the data, while still keeping the input format transparent for human inspection, and can cope with complex models needed for the combination of a large number of channels with non-trivial correlated uncertainties. In 2012, Petrucciani extended the combination framework to include measurement of the properties of a Higgs-like resonance. He has led the development of first benchmark models for the measurement of Higgs boson mass, coupling and spin-parity. Since 2011, Giovanni has played the anchor role in coordinating CMS results on the combinations of all SM, fermiophobic and fourth-generation Higgs searches. Petrucciani, Sharma and Korytov wrote the CMS paper on the combination of SM Higgs searches with 2011 data which was published in Phys. Lett. B 710 (2012). They also co-wrote the confer-
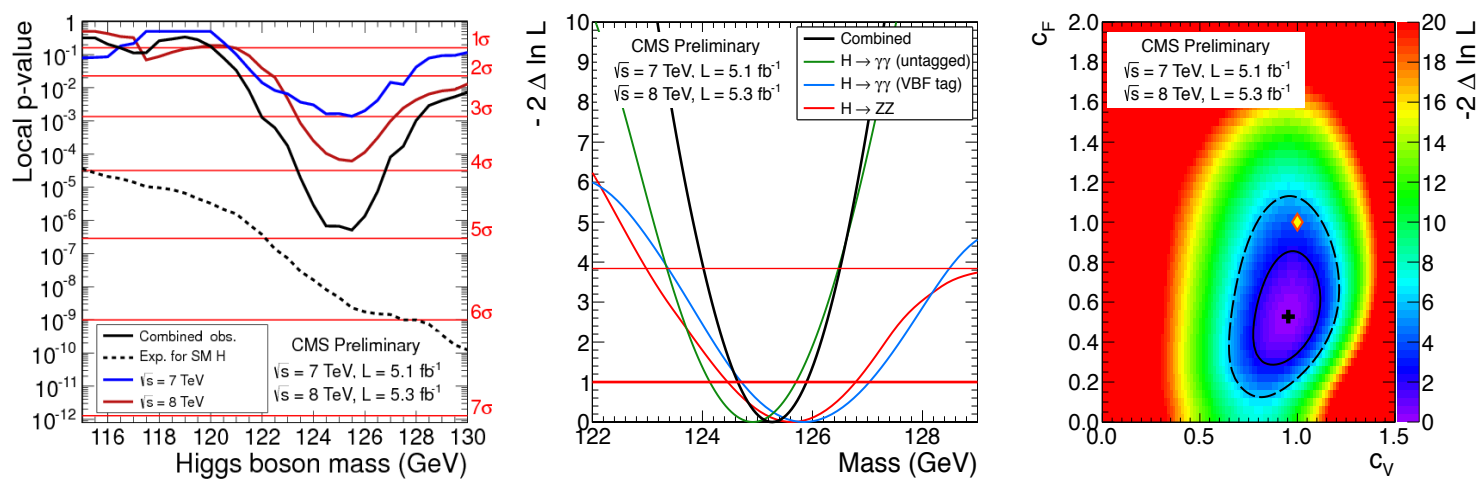

Figure 3.4: Properties of the observed boson: Significance of observation (left), invariant mass (center) and the couplings for fermions and bosons (right) normalized to SM expectation.

ence note (CMS PAS HIG-12-020) on the "Observation of a new boson with a mass near 125 GeV" which was released as a preliminary result for ICHEP'12. It reported (Figure 3.4) the significance of the observation, the mass of the observed boson from the combination of the $4 \ell$ and $\gamma \gamma$ channels; the compatibility with the custodial symmetry; and the compatibility with the SM Higgs boson hypotheses for fermion and boson couplings from a combined analysis of all modes. This note was the basis of the CMS paper "Observation of a new boson at a mass of $125 \mathrm{GeV}$ with the CMS experiment at the LHC" submitted to PL(B) on Aug 31st, 2012.

3.1.3 CMS Tracker \& Charged Particle Tracking: Our group has been involved in the Si-strip Tracker and charged particle reconstruction since 2007. Postdocs Mangano and 
Petrucciani are two of the CMS tracking experts. Mangano served as the co-convener of the Tracking group during the crucial commission phase (2009-10). He is one of the two main authors of the "Combinatorial track finder" used by CMS due to its flexibility, efficiency and speed. Mangano's numerous contributions to core tracking software include (a) implementation of tracking algorithms in CMSSW framework, (b) Si-strip based trajectory seeding (c) Bi-directional (inside-out and outside-in) trajectory builder (d) iterative tracking to increase low Pt track reconstruction efficiency, (e) algorithm for fake track rejection and (f) optimization of track fit in the non-uniform solenoidal field. Petrucciani is in charge of the implementation and optimization of tracking software in CMS online \& offline event reconstruction. He is also an expert in Muon reconstruction. Student Vartak is in charge of the validation of tracking performance in each CMS software development cycle. The validation tools that Vartak developed, provide an extensive repository of diagnostic plots to monitor changes in tracking efficiency, track parameters, fake rate and other observables which measure the efficacy of online, offline and fast-simulation software. Vartak serves as an on-call Tracker DAQ expert and will participate in tracker activities at P5 during LS1. He is also involved in analyzing time delay in tracker response and the calibration data. Lebourgeois contributed to the Tracker commissioning and DQM and investigated impact of several Tracker failure modes on Higgs physics.

Charge particle tracking in CMS has worked seamlessly since the very first LHC collisions with a high track reconstruction efficiency, low fake rate and close-to-design resolution in the measurement of track parameters. While important layers of sophistication have been added, the performance in general was quite stable till Fall'11. Complications arose when the in-time pileup produced by LHC grew much larger than originally expected. The 2012 run projected a further increase in the average pileup by at least a factor of two and with large tails. Given the limited CPU and memory resources available for prompt event reconstruction, CMS set up a task force to react quickly to significantly decrease the average time \& memory spent in reconstructing high-pileup events. Since tracking is the most resource consuming part of CMS event reconstruction, Mangano and Pertucciani led a team of 5 experts who focussed on the modification and optimization and speed up of the tracking algorithm. Mangano optimized the key algorithms used in several steps of the track reconstruction sequence to reduce their memory footprint without impacting their functionalities. The largest speedup was obtained by optimizing track seed filtering. Track seeding is the earliest step in track reconstruction and due to the combinatorial nature of the algorithm, increases sharply with pileup. Petrucciani developed a seed filtering algorithm based on compatibility between the hypothesized particle trajectory and the shape of charge deposit in the Pixel tracker. The early rejection of spurious track seeds arising from combination of hits from different particles allowed a $30 \%$ reduction in the overall time for track reconstruction with negligible loss in efficiency. Another gain came from elimination of "looper" hits. Events with pileup contain a large number of tracks with $\mathrm{Pt}<1 \mathrm{GeV}$ which, in the $3.8 \mathrm{~T}$ solenoidal field, spiral till they escape thru the endcap or are absorbed in the tracker material. Each such "looper" produces in excess of 70 hits in the innermost tracker layers. Since the tracking algorithm was originally optimized for moderate-Pt tracks (which go almost straight thru the tracker volume), only a small fraction of the looper trajectory were reconstructed. The unassociated hits from the loopers add to the pool of hits from which further track seeds are generated and unnecessarily increase event reconstruction time. Mangano made major improvements in the tracking algorithm so as to fully reconstruct trajectory of the loopers with high efficiency and then remove the associated hits from further consideration. In addition to the targeted speedup in time for reconstruction, this improvement also led to a $\approx 25 \%$ reduction in the rate of duplicate tracks caused when the true trajectory of even a high Pt track is broken up into two or more segments. 


\section{Chapter 4: Profs Würthwein and Yagil}

Profs Würthwein and Yagil operate a joint research group on CMS that spans a broad physics program and diverse technical contributions to CMS in software, computing, trigger, and tracking. The people in this group who have received partial support from the existing grant within the last 3 years are Dr. G. Cerrati, Dr. D. Evans, Dr. J. Mülmenstädt (left in 2010), Dr. S. Padhi, Dr. Y. Tu, and graduate students W. Andrews (grad. 2012), F. Golf (grad. 2011), R. Kelley, I. MacNeill, V. Welke, and J. Yoo.

\subsection{CMS Physics Analysis during the last 3 years}

Professors Würthwein and Yagil have a common physics objective at CMS, to search for new physics in final states with two or more leptons.

In our last three-year proposal of 2009 we spoke of our plans for startup data, development and commissioning of tools and hopes of Physics papers to be written. We stated that our research goals revolve around the search for new physics in the dilepton final state with or without jets, with or without $E_{T}$. We said, we would start by reestablishing the Standard Model by measuring the $t \bar{t}$ and WW cross-sections in the dilepton final state, and explore from there.

In the past three years, as reported in the corresponding progress reports, we have done just that:

- Using early (2010) data we played a leading role in the commissioning of MET and also contributed to the debugging and commissioning of various other objects. We were responsible for the first publications of the top pair[44][45] and WW[46] cross-sections. We also pioneered the searches for SUSY in the Opposite-Sign[47] and Same-Sign[48] di-lepton final states and wrote the early CMS papers on them with the $35 / \mathrm{pb}$ of 2010 data available.

- During 2011 we maintained two main drives: The search for the Higgs in WW[50] and ZZ[52] to $l \nu l \nu$ and searches for New Physics (NP). These searches expanded the reach of the early ones by using the full $20115 / \mathrm{fb}$ dataset, and refined them, like adding b-tags to the SameSign[58] search for SUSY, that increased its sensitivity dramatically.

- Using the $8 \mathrm{TeV}$ data CMS has observed a new boson and is still searching for evidence of NP. Currently we are engaged in trying to measure its characteristics and are continuing the search for NP. The focus of these searches is targeted at the trendy "Natural SUSY" [59] and we hope that by the end of the $8 \mathrm{TeV}$ run we will be able to observe or limit it significantly. Our ongoing work and physics plans will be described in Section 4.2.

Sadly, the only thing we "failed" to do, out of our proposal, was discover any new physics.

In the following, we will try to give a brief overview of some of the physics analyses we have completed. All of these analyses have been submitted for publication, or are published. Ongoing analyses as well as analyses completed and presented at ICHEP are discussed in Section 4.2.

NOTE: All of our analyses are done in close collaboration with Claudio Campagnari (UCSB), Lothar Bauerdick and Kevin Burkett (FNAL), and their post-docs and students. Our work related to Higgs searches includes additional collaborators from MIT. Section 4.1 briefly describes this body of work. During the ending three-year period 2009-2012, that spans the LHC startup, through the Higgs discovery and to date, CMS has published (or placed on the arxiv) 160 papers. Out of these, our group played a significant role or lead outright 13 of them. They are listed in Table 4.1. 
Table 4.1: List of our published CMS Physics papers - to date.

\begin{tabular}{|c|c|c|c|}
\hline$\#$ & Paper name/subject & Journal & Cites \\
\hline 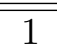 & top $\mathrm{x}$-section $3 / \mathrm{pb}$ & PLB 695:424-443,2011 & 72 \\
\hline 2 & top $\mathrm{x}$-section $36 / \mathrm{pb}$ & JHEP 07 (2011) 049 & 45 \\
\hline 3 & WW x-section $36 / \mathrm{pb}$ & PLB 699 (2011) 25 & 66 \\
\hline 4 & Search for new physics in Opposite Sign di-leptons 34/pb & JHEP 6 (2011) 26 & 40 \\
\hline 5 & Search for new physics in Same Sign di-leptons 35/pb & JHEP 1106:077,2011 & 41 \\
\hline 6 & Search for Same Sign top pairs & JHEP $8(2011) 5$ & 43 \\
\hline 7 & Search for Higgs to WW (in $l l \nu \nu$ final state) & PLB $710(2012) 91$ & 61 \\
\hline 8 & Search for Higgs to ZZ (in $l l \nu \nu$ final state) & JHEP $1203(2012) 040$ & 21 \\
\hline 9 & Search for t' pair production & arxiv.org/abs/1203.5410 & 18 \\
\hline 10 & Search for new physics in Opposite Sign di-leptons $5 / \mathrm{fb}$ & arxiv.org/abs/1206.3949 & 4 \\
\hline 11 & Search for new physics in Z+Jets+MET $5 / \mathrm{fb}$ & arxiv.org/abs/1204.3774 & 6 \\
\hline 12 & Search for N.P. in Same Sign di-leptons 5/fb & PRL 109, $071803(2012)$ & 8 \\
\hline 13 & Search for N.P. in Same Sign di-leptons w b jets & arxiv.org/abs/1205.3933 & 14 \\
\hline
\end{tabular}

\subsubsection{Measurement of the top cross-section in the dilepton final state:}

This was the first measurement of the top quark cross section at the LHC. It was performed based on the first $3 / \mathrm{pb}$ of data collected by CMS, presented in ICHEP that year and published in Physics Letters B [44]. This measurement is part of Frank Golf's Ph.D. thesis.

In this early measurement we used very loose lepton ID cuts to maximize the acceptance to enable as timely an observation of the top quark, as possible. We required two oppositesign, isolated leptons with $P_{T} \geq 20 \mathrm{GeV}$. To reject Drell-Yan background we veto same-flavor di-lepton pairs that are within $10 \mathrm{GeV}$ of the $\mathrm{Z}$ mass and also required that such events have more than $30 \mathrm{GeV}$ of $\not_{T}$. For electron-muon pairs, we loosened the $\mathscr{E}_{T}$ requirement to $20 \mathrm{GeV}$.

Dileptons from top events will be accompanied by at least two hadronic jets from the hadronization of the two b quarks. Therefore, candidate events were required to have at least two jets with $p_{T} \geq 30 \mathrm{GeV}$ and within $\eta \leq 2.5$. The resulting Njets distribution is shown in Fig 4.1 (without the two jet requirement).

Taking into account the data yield, the background esti-

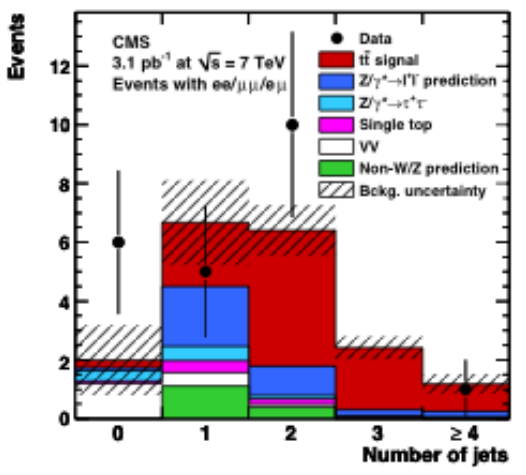

Figure 4.1: Number of jets in events passing all dilepton selection criteria, compared to signal and background predictions. The hatched bands reflect the uncertainties on the background predictions. mation, branching fraction, signal acceptance, integrated luminosity, and all associated statistical and systematic uncertainties, the top-quark pair production cross section was measured to be: $194 \pm 72$ (stat.) \pm 24 (syst.) \pm 21 (lumi.) pb [44]. We repeated this measurement one more time, based on $35 / \mathrm{pb}$ of the full 2010 data set and obtained: $168 \pm 18$ (stat.) \pm 14 (syst.) \pm 7 (lumi.) pb [45].

4.1.2 Search for Higgs in the WW to $\mathrm{l} \nu \mathrm{l} \nu$ final state: This channel has the most sensitivity for SM Higgs in the range 130-200 GeV, but is highly non-trivial due to the nature of the final state signature; two leptons, missing transverse momentum (MET) and no jets. This signature suffers from many background sources, lacks a clean "smoking gun" mass peak, and hence reduces to a counting experiment based on a comprehensive understanding of many background sources. 
There were two independent analyses in CMS of this channel. Our group (with Campagnari, Bauerdick, Burkett, Paus, and Klute) was one of the two.

At the start of the data taking, and recognizing the key importance of the triggers, our group took responsibility for all the triggers used in this analysis. This included the triggers used to collect the signal samples (in the ee, $\mu \mu$ and $\mathrm{e} \mu$ channels) as well as samples used for estimating all the backgrounds (fake rates) and signal efficiencies. This required continuous involvement in the development and maintenance of the trigger menu used by CMS and the derivation of the relevant fake rates and efficiencies, throughout the run.

The main background sources for this analysis are:

- WW - An irreducible background, identical in composition to the signal final state. Some kinematical differences for certain Higgs masses.

- ttbar - Huge cross-section and in the case where both b-jets missed, similar to signal.

- Drell-Yan - Huge cross-section and with fake MET mimics signal's signature. Must understand MET very well.

- W+jets - Has one real lepton and real MET, a fake lepton from a jet produces a signal-like signature.

There are two related goals in dealing with these BG sources: first is to reduce them as much as possible, and as importantly, estimate reliably the residual background left - after all cuts. Our group has created the procedures used in the final CMS 2011 publication for all of the above.

We have also led the effort to stabilize the analysis and the various procedures against "deteriorating" running conditions related to the continuous increase in instantaneous luminosity - the increase in multiple interactions. We developed and deployed in the analysis the first set of tools used for controlling fake MET, recovering some of the efficiency loss in isolation cuts due to pile-up and others. These became CMS standards in many analyses within the Higgs group as well as in other analysis groups.

Our analysis has been published on its own using the full 2011 data set [50] and excluded the SM Higgs in the mass range of: $129-270 \mathrm{GeV}$ at the $95 \%$ C.L.

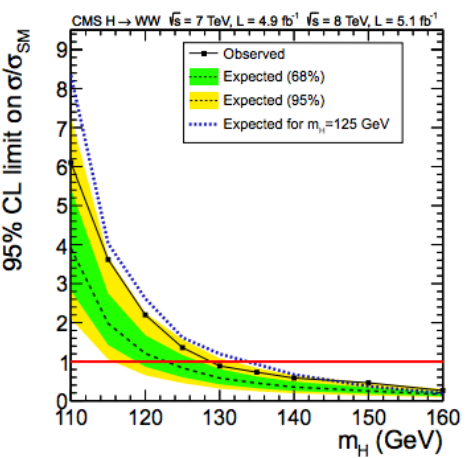

Figure 4.2: Higgs to WW

We updated this analysis with the first $5 / \mathrm{fb}$ of $8 \mathrm{TeV}$ data, and limit from [51] the combined 7 and $8 \mathrm{TeV}$ analysis was published as part of the CMS "observation" paper [51]. The 95\% CL expected and observed limits for the combination of the 7 and $8 \mathrm{TeV}$ data sets are shown in Figure 4.2. A broad excess is observed. This excess is consistent with a $125 \mathrm{GeV} \mathrm{SM}$ Higgs boson and this channel's mass resolution. The dotted curve in Figure 4.2 shows the median expected limit for a SM Higgs boson with mass $125 \mathrm{GeV}$ as can be seen, the observed cross-section is slightly lower than the expected under the hypothesis of a SM Higgs. The expected signicance for a SM Higgs of mass $125 \mathrm{GeV}$ is $2.4 \sigma$ and the observed signicance is $1.6 \sigma$.

4.1.3 Search for Higgs in the $\mathrm{ZZ}$ to $\mathrm{ll} \nu \nu$ final state: This channel has good sensitivity to an intermediate Higgs mass in the range $250-500 \mathrm{GeV}$. It relies on the Higgs decay to two $Z$ bosons where one of them decays to two leptons and is fully reconstructed, and the other decays to two neutrinos and is invisible, The signature is thus, $Z$ and MET, where jets may come only from Initial State Radiation (ISR).

There were two independent analyses in CMS of this channel. Our group (with Campagnari, Bauerdick, Burkett, Paus, and Klute) was one of the two.

The background sources affecting this analysis are similar to those in the Higgs to WW, however, their relative importance is very different. $t \bar{t}$ and $\mathrm{WW}$ are dealt with relatively simply, by the socalled opposite flavor subtraction method (since Z does not decay to e- $\mu$, one can estimate the $t \bar{t}$ 
and WW and subtract their contributions this way). The main concern is $\mathrm{Z}+$ jets where a badly measured jet(s) can cause significant MET. Our group developed the procedure used to estimate this background and assess the systematic uncertainty on it.

Using the 2011 data set, this analysis [52] excluded the SM Higgs in the mass range of: $270-$ $440 \mathrm{GeV}$ at the $95 \%$ C.L. We did not repeat this analysis with the $8 \mathrm{TeV}$ data set.

4.1.4 WW cross section measurement in the dilepton final state: Given that the WW process is the largest irreducible background to the Higgs in the same channel, we made it a priority for early data taking. We have produced the first measurement of this final state using the 2010 data-set [46].

Figure 4.3 offers a simple overview of this analysis. It clearly shows the large $t \bar{t}$ contributions associated with jets, the large Drell-Yan backgrounds and the signal shape and location in the Njet distribution and the dilepton mass. These are the main ingredients that need to be controlled and understood to extract the WW signal.

Preliminary public results exist for both $5 / \mathrm{fb}$ of 2011 , $7 \mathrm{TeV}[66]$ and $5 / \mathrm{fb}$ of $2012,8 \mathrm{TeV}$ [67], and a draft paper using the full 2011 data-set is under CMS internal review
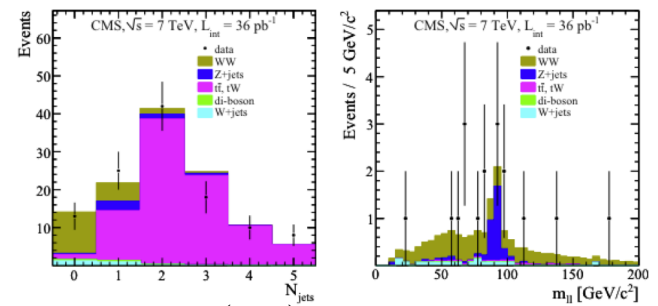

Figure 4.3: (left) ${ }^{\text {jes }}$ Jet multiplicity after all WW selection criteria, except top and jet vetoes, (right) Dilepton mass for events passing final selection, except Z mass veto. at the time of this writing. It will include a limit on anomalous triple gauge coupling. We continue this analysis as part of the $\mathrm{H}$ to WW effort, as well as a starting point for new physics searches.

4.1.5 Search for heavy, top-like quark pair production in the dilepton final state: Requiring a baseline selection of two leptons with pT $>20 \mathrm{GeV}$, exactly two b-tagged jets with $\mathrm{pT}>30 \mathrm{GeV}$, and $\mathrm{MET}>50 \mathrm{GeV}$ selects a sample of $\sim$ 2600 events that is $\sim 97 \%$ pure top anti-top quark pairs, out of the $5 / \mathrm{fb}$ CMS data from 2011. Having selected exactly two b-tags, we then compute the minimum invariant mass combination between the b-tags and the two leptons, $M_{l b}^{\text {min }}$, and require that to be $>170 \mathrm{GeV}$. Figure 4.4 shows the $M_{l b}^{\min }$ distribution in data and MC. This eliminates essentially all the background from top decays, resulting in an estimate of $1.8 \pm 1.1$ events from standard model sources in $5 / \mathrm{fb}$ of CMS data at $7 \mathrm{TeV}$ pp collisions. We observe one event, and calculate upper limits on the production cross section as a function of $t^{\prime}$ mass. We exclude $t^{\prime}$ masses below $557 \mathrm{GeV}$ [54].

\subsubsection{Search for SUSY-like signature in the op- posite-sign dilepton final state outside the $\mathrm{Z}$ peak:} Requiring a baseline selection of two leptons with pT $>20 \mathrm{GeV}$

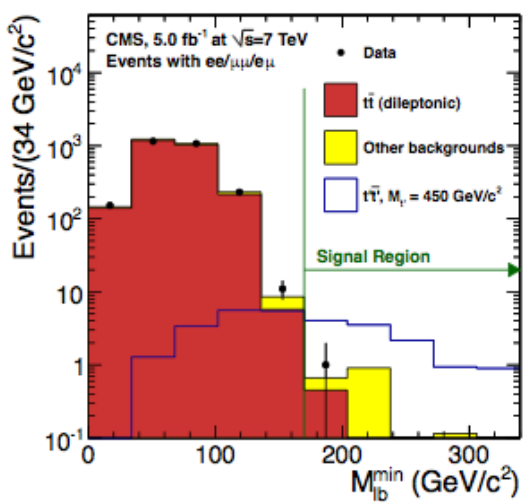

Figure 4.4: Comparison between data and simulated background for $M_{l b}^{\min }$. The expected distribution for $t^{\prime}$ pair production at a $t^{\prime}$ mass of $450 \mathrm{GeV}$ is also shown. and $10 \mathrm{GeV}$ respectively, MET $>50 \mathrm{GeV}$, and $\geq 2$ jets of $\mathrm{pT}>30 \mathrm{GeV}$ each, and a $\mathrm{Z}$ veto for same flavor leptons reduces the 5/fb CMS data from 2011 to a sample of $\sim 11000$ events that is $\sim 84 \%$ pure top pair production. After verifying that one dimensional distributions of kinematic variables, e.g. HT, MET, dilepton pT, \# of jets, are consistent between data and MC, we search for new physics in the tails of MET and HT. HT here refers to the sum of the pT of all jets with pT > $30 \mathrm{GeV}$ in the event. According to the $\mathrm{MC}$, even these tails are dominated by top pair production where the MET is attributable to the di-neutrino $\mathrm{pT}$. To estimate this background we thus use the dilepton $\mathrm{pT}$ in data to predict the MET distribution for a given HT range. Figure 4.5 compares 
this prediction with the actual for $\mathrm{HT}>300 \mathrm{GeV}$, showing excellent agreement. No evidence of new physics is seen, and we interpret our result in terms of constraints on the CMSSM parameter space. This analysis was done first using 35/pb of 2010 data [47] and repeated using the full 2011 data set of $5 / \mathrm{fb}[55]$.

\subsubsection{Search for SUSY-like signature in the op- posite-sign dilepton final state inside the $\mathrm{Z}$ peak:} Our work here is leading to two publications [56][62]. Both have a common baseline selection of ee and $\mu \mu$ pairs with $\mathrm{pT}>20 \mathrm{GeV}$ and an invariant mass within $10 \mathrm{GeV}$ of the $\mathrm{Z}$ mass, and $\geq 2$ jets of $\mathrm{pT}>30 \mathrm{GeV}$. The first paper is a generic search for new physics with $\mathrm{Z}$ plus MET plus $\geq 2$ jets, while the second is a dedicated search for weakly pair-produced chargino/neutralino in the WZ and ZZ plus MET final state. Figure 4.6 compares the MET distributions in the two searches side by side. It is clear from this figure that we see no evidence for new physics in either of the two analyses. Comparing the two MET distributions shows a large reduction in both top and $\mathrm{Z}+$ jets backgrounds in the second (more narrowly targeted) analysis. Top background is reduced by applying a b-tag veto, and backgrounds are reduced further by requiring two of the

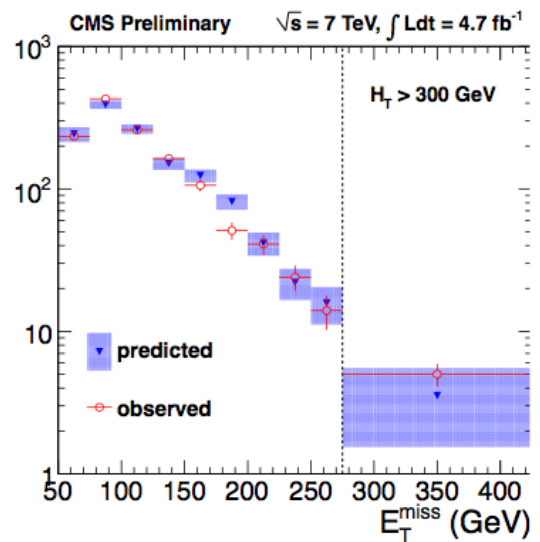

Figure 4.5: Comparison of predicted and actual MET distribution for $\mathrm{HT}>300 \mathrm{GeV}$. jets to have an invariant mass consistent with the $\mathrm{W}$ or $\mathrm{Z}$ boson in addition to the selections chosen in the first analysis. As a result, the $\mathrm{Z}+$ jets bkg becomes dominant up to larger MET values than in the generic analysis.

The tails of the MET distribution, and thus the $\mathrm{Z}+$ jets background in both of these analyses is estimated by measuring MET templates as a function of HT and number of jets in photon plus jets events in data. The top background is measured using an e $\mu$ control sample.

\subsubsection{Search for SUSY-like signature in the Same-Sign dilepton final state:} Our work here has lead to four publications. Using the 35/pb of 2010 data we performed a SUSY search in the Same-Sign dilepton sample. This was a "generic" search that looked at Same-Sign dileptons in combinations with jets and Missing ET [48]. In addition we performed a specific search for Same-Sign top [49], inspired by the reported CDF Forward-Backward anomaly. These searches are part of Frank Golf's Ph.D. thesis.

Using the $5 / \mathrm{fb}$ of the 2011 run, we repeated the 2010 "generic" search. While in 2010 we restricted ourselves to only high pT $(20 / 10 \mathrm{GeV})$ ee, $\mathrm{e} \mu$, and $\mu \mu$ final state, in 2011 , we expanded the search to muon (electron) momenta of $5 \mathrm{GeV}(10 \mathrm{GeV})$. The basic search
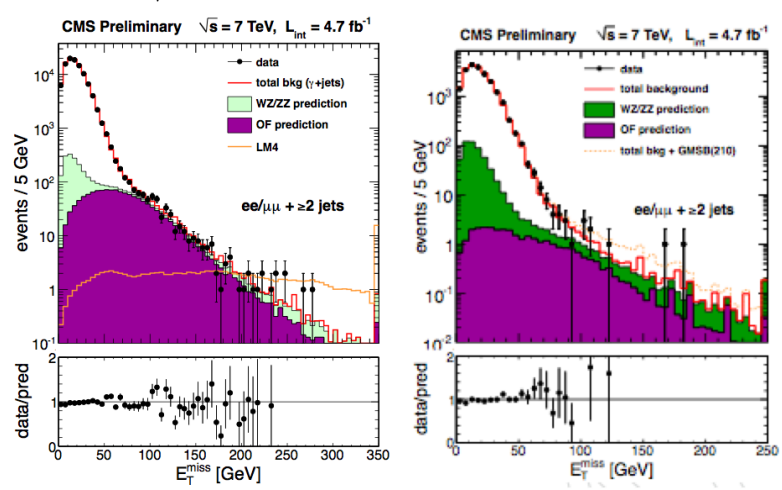

Figure 4.6: MET distribution in $\mathrm{Z}+\mathrm{MET}+$ jets without extra b-tag veto and dijet invariant mass cut (left), and with these requirements (right) strategy and techniques are the same as those used with the 2010 data, however the details in fake rate measurements, and sensitivity to rare standard model backgrounds changed significantly. No evidence of new physics was observed, and the final 2011 sensitivity achieved in the CMSSM plane at large squark masses is competitive with the CMS hadronic and single lepton analyses. Given the null results in the 2010 SUSY searches by ATLAS and 
CMS, the interest in SUSY has migrated towards phenomenology motivated by "naturalness" arguments[60]. This motivates final states with four W's and 2-4 b-quarks plus 2 LSP's. Therefore, we proposed and executed an analysis focused on final states with at least two same sign $\mathrm{W}$ decays to $\mathrm{l} \nu$ and $\geq 2$ b-tags. The b-tag requirement eliminates the dominant backgrounds, resulting in a factor 35 smaller background as compared to the more inclusive search. As in all other searches at the LHC, we see no sign of new physics. In our paper [58], we provide 6 different model interpretations, and show that we can exclude gluino pair production up to a gluino mass of $\sim 800 \mathrm{GeV}$ irrespective of the details of the decay chains into sbottom and stop as long as the W's in the final states are on-shell, and at least two of the b-quarks produced have typical energies above $\sim 40 \mathrm{GeV}$. In addition, we excluded sbottom pair production up to a sbottom mass of $\sim 340 \mathrm{GeV}$, and have significantly improved upon our previous Same-Sign top pair production limit [49].

Figure 4.7 shows the yield for one search in the HT vs MET plane. It shows clearly the dramatic difference in background between the two analysis strategies. For the kinds of SUSY models considered, the $\times 35$ reduction of background comes at a price of a $\times 2$ drop in efficiency. We have convinced CMS that this change in strategy for the primary results on the same sign dilepton search is the right path forward in 2012.

\subsection{Physics}

\subsubsection{Prof Würthwein - Highlights:} Prof. Würthwein's physics interests for the past 5 years have been in physics with leptons, MET, and jets. This started with work at CDF, leading to the observation of WZ [39] and ZZ [40] production in 2007/08, and development of CDF's Higgs search strategy in the

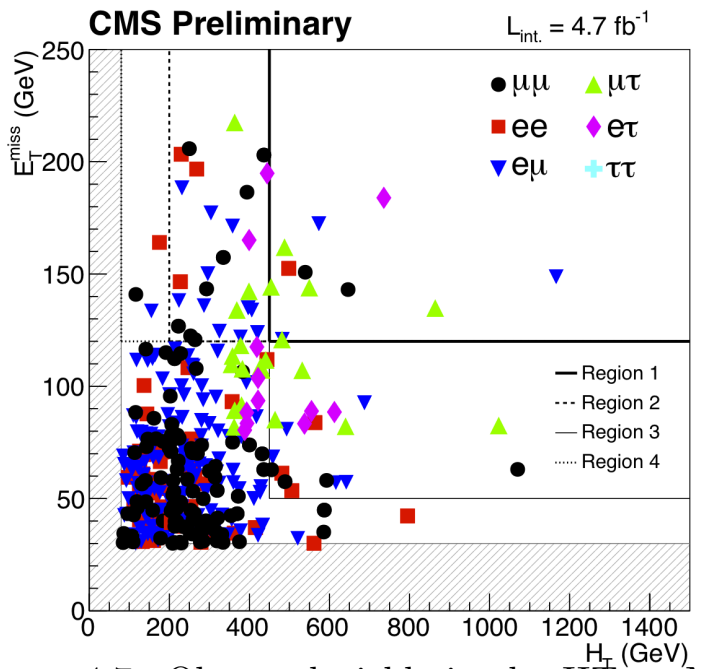

Figure 4.7: Observed yields in the HT vs MET plane for our "generic" same-sign dilepton analyses (2011). WW final state [41][42] in 2008/09, and has expanded since to include a wide range of topics as described in Section 4.1.

Würthwein's technical contributions to CMS are mostly in computing, and to a lesser extent in MET commissioning, where he was the JetMet representative to the "Anomalous Signals in the Calorimeter Task Force" in 2010, and the CMS generator group, where he is supporting S. Padhi as co-lead of the group for 2012/13.

In computing, Würthwein was part of CMS computing management from 2006-2011, co-leading the Global Computing Commissioning (2006-2009), and Analysis Operations (2009-2011). He handed over managerial responsibilities to James Letts (UCSD), who co-leads what is now called "Physics Support" (2012/13) in CMS. In addition, Würthwein was the founding chair of the Open Science Grid (OSG) in 2004, and is since one of the members of the OSG Executive Team, and a Co-PI of the OSG grant.

The following provides a brief summary of the highlights of Würthwein's technical contributions to CMS in computing.

Global Computing Commissioning: Würthwein's group commissioned the global data transfer network of CMS, designed and developed some of the core grid tools CMS uses for Analysis as well as reconstruction and simulation [43], commissioned the grid infrastructure in general, and worked with others in CMS to develop the basic metrics, procedures, and policies used by CMS 
to evaluate Tier-2's, and assign credit to the institutions that operate them. Würthwein has been the CMS contact within OSG for all planning and coordination purposes at the management level since $\sim 2005$.

Infrastructure Operations: Würthwein's group has been operating a Tier-2 center for CMS since 2005. In 2009, we added responsibility for CRAB server operations. CRAB servers are the CMS equivalent of Panda for ATLAS, SAM for D0, or CAF for CDF, i.e. the infrastructure the physicists in the experiments use to do large scale data analysis, and generate private Monte Carlos. Around the same time, we also added responsibility for glideinWMS operations, a grid middleware layer used not just by CMS but also an ever increasing number of other sciences. As of today, roughly $1 / 4$ of the CPU on OSG is consumed by sciences other than HEP, and essentially all of them use the glideinWMS infrastructure operated by our group. Most recently, we added "Any data, Anytime, Anywhere" functionality which does as the name says, i.e. allowing CMS physicists to access any data that is on disk at any US Tier-2 or the FNAL Tier-1, from a root prompt, or CMSSW, or fireworks display from anywhere with an internet connection. Since 2009, we manage a global disk cache across all CMS Tier-2 centers that hosts all standard Primary Datasets of CMS, and a set of standard model background Monte Carlo samples for the entire experiment. What exactly is considered "standard" is decided in collaboration with Physics Management, with computing providing usage metrics, and being responsible for appropriate placement across the Tier-2 centers.

Würthwein's future physics focus is in Higgs properties, especially measuring the spin of the observed resonance at $125 \mathrm{GeV}$, and the search for "Natural SUSY" and related phenomena, as explained in Section 4.2.

4.2.2 Prof Yagil - Highlights of Technical Contributions: Yagil's contributions to CMS were in the areas of software and trigger. Below we list the main ones.

- Initiated the re-engineering of CMS software, led the design team that introduced the CMS Event Data Model (ROOT browse-able) and led the initial implementation of CMSSW and migration from the old framework.

Prior to that, CMS relied on an Objectivity-based event data model that caused severe consequences for data access and usability e.g. weak concept of event. The software framework and reconstruction code relied exclusively on reconstruction on demand for scheduling of execution. This had severe implications for HLT and offline reconstruction in terms of reproducibility, debugging ability and so on. The new framework and event data model are one of CMS competitive edges with respect to ATLAS.

- Initiated and created the CMS event display - Fireworks. It replaced the legacy IGUANA just in time for data taking.

During our group's preparation for the first CMS data taking run, we ran into the problem that there was no usable visualization tool in CMS. We faced the need to understand reconstruction and analysis issues, chase bugs in MC, geometry. Finding no existing solution and in coordination with CMS management, Yagil proposed to develop a physics-analysis event display. We were charged to do so a few months before data taking and worked very intensively to create and deploy it in time for the LHC startup. Currently it is being used in the control room to monitor data quality and to produce online pictures sent world-wide to all CMS Centers. Its main usage however, remains as an analysis tool were it is used to debug and develop most analyses as well as the reconstruction code.

- Have been a member of the CMS Physics Office (10/11), focused on triggers. Mostly in the di-lepton final state (key for Higgs, SUSY as well as TOP and EWK analyses). Designed the di-lepton trigger strategy for CMS used in all high PT di-lepton physics analyses. These triggers are essential in the search for a light Higgs in the WW channel, where one of the Ws is 
off-shell and hence the lepton is low PT (down to $10 \mathrm{GeV}$ ). This included the introduction of a set of asymmetric triggers for $e e, e \mu$ and $\mu \mu$. A consistent family of triggers was developed and deployed for signal collection along with a set of utility triggers for efficiency as well as fake rate measurements.

In addition, Yagil was a member of 9 analysis review committees (ARCs) and has chaired 6 out of them.

4.2.3 Trigger: During the initial data taking the CMS trigger menu and procedures associated with it underwent a rapid evolution. Rapidly changing machine conditions and the dramatic increase in instantaneous luminosity presented multiple challenges. During the better part of the 2010 data taking CMS placed continuous catch-up to be able to take relevant data.

One of the consequences of these rapid changes was that triggers would frequently change content (cut values etc.) but retain their name. There was no way to tell given a trigger name what and how was precisely the selection applied at the High Level Trigger (HLT) for a given data sample. Yagil proposed a versioning system that was adopted and followed since then.

To improve the situation for the 2011 run, the CMS Physics Office took upon itself to define an overall set of priorities and interface the various physics analysis and object groups with the Trigger group, Led by Prof. Acosta (U. Florida). Yagil's role in this context was the dilepton triggers in general and specific focus on the e/g physics objects related triggers.

This issue became even more pressing when the Higgs was excluded at $160 \mathrm{GeV}$. The implication for the search in the most abundant final state - $H \rightarrow W W$ was that we had to allow for one of the W's to be off-shell and hence the dilepton triggers needed to have one of the leptons at low PT. One of the most insidious background for the Higgs search in the WW channel at low Higgs mass is $\mathrm{W}+$ Jets where one of the Jets produces a fake lepton. This background must be estimated using data, in the signal sample. The challenge was to introduce efficient triggers for signal collection within a reasonable trigger budget (rate) and sufficiently large "sideband" that will allow for the essential in-situ background estimate. Yagil led the design of a complete suite of dilepton triggers including signal triggers in all the relevant combinations of ee, e $\mu, \mu \mathrm{e}$ and $\mu \mu$ final states with an asymmetric topology, i.e $17 \& 8 \mathrm{GeV}$ respectively with carefully chosen ID and isolation requirements to meet the budgets in a stable way through the 2011 and 2012 runs.

4.2.4 Tracking: UCSD has a strong tracking tradition in CMS and elsewhere. Post Doc Cerati has been appointed as the next convener of the tracking group. Post docs Mangano (extracking convener and key developer) and Petruccianni (key developer) in Sharma's group have been key contributors to the CMS tracking effort.

4.2.5 Fireworks: Physics Analysis Event Display: Yagil initiated a new project within the experiment to construct an event visualization tool. This project was a direct result of the preparation work done within our group, prior to data taking startup. It got started to solve a series of problems we encountered in our analysis work and that we could not address using the existing, standard CMS tools.

Initially it was meant to be purely an analysis tool. However, it was such a success that the collaboration asked it to be deployed also as a monitoring tool in the control room. Later on, we were asked to add functionality to explore and display raw data and low-level detector information. These things were done, and Fireworks was used at the startup of the LHC in Sep 08 as one of the two event displays of CMS. However, it quickly became the tool of choice by people doing analysis and the legacy tool was later retired. Fireworks has become the official CMS event display analysis tool and is being used extensively since startup in the control room, media events and CMS analyses. 
There has been two major developments during the last year:

- A geometry browser has been created and integrated that allows checking the details of detector description in detail.

- An implementation of a full-framework-resident event display that enables much deeper debugging of the CMS reconstruction software.

4.2.6 Computing: The UCSD group has long played a leadership role in CMS computing. Branson \& Fisk started operating a CMS Tier-2 center prototype in 2000. In 2005, Würthwein took over when it became one of the first four US-CMS Tier-2 centers. Throughout the years, we have been quite successful in finding additional funding to support our work on computing in support of CMS, and thus leverage the support via this grant to do a lot more.

Computing effort is mostly funded outside the scope of this DOE proposal, through a mixture of DOE and NSF sources. In the past, some of our post-docs (Evans, Padhi) and students (MacNeill, Andrews, Yoo) have done their CMS service work in the context of the operations activities listed below, including the occasional development projects in support of them. We expect this to continue in the future.

Our focus continues to be on issues most immediately relevant to physics analysis, both in terms of the infrastructure services we operate, e.g. Tier-2 center, CRAB, glideinWMS, as well as the infrastructure software R\&D we engage in.

Our present operational responsibilities in the CMS collaboration are as follows:

- Operations of the UCSD Tier-2 center. The Tier-2 is officially hosting analysis efforts for three "physics" groups - EWK, SUSY, and e-gamma - supporting CMS Monte Carlo production, and providing support for roughly 80 CMS physicists from UCSB, UCSD, UCR, and some Fermilab collaborators. This "local community" is storing 2 Million files in our storage system as of August 13th, 2012. Roughly $10 \%$ of all physics publications of CMS so far include substantial contributions that depended on ntuples produced by our "local community" and stored here.

- Operating the server infrastructure used by CMS to submit data analysis jobs to the grid. The UCSD group operates this service on hardware at CERN, UCSD, and Bari (Italy).

- Operating the glideinWMS infrastructure that CMS, as well as a host of other sciences use to manage their job submissions on the grid. i.e. CRAB and the WMAgent systems used by dataOps for simulation and reconstruction both are CMS specific software layers that submit into a CMS independent layer underneath that makes the grid look like a single batch system across all grid sites. UCSD leads an operations team comprised of effort at UCSD, FNAL, and CERN, and operates services deployed by UCSD, FNAL, CERN, and the OSG Grid Operations Center at Indiana University.

- Managing a distributed cache of presently 6 Petabytes of data across 41 Tier-2 centers worldwide. This cache hosts all the commonly used data and Monte Carlo samples of the experiment. This includes developing and implementing policies in collaboration with physics management for what data should be distributed into this space, how many replicas are required, which data goes where, etc.

- The UCSD group assembles performance metrics on data analysis in CMS worldwide. This includes weekly reports on resource consumption, failure rates and causes, dataset utilization.

- Operations of the global monitoring infrastructure for the "Any data, Anytime, Anywhere" project in support of CMS. 


\section{Chapter 5: Bibliography and References Bibliography}

[1] S. Chatrchyan et al. [CMS Collaboration], "Observation of a new boson at a mass of $125 \mathrm{GeV}$ with the CMS experiment at the LHC," [arXiv:1207.7235 [hep-ex]].

[2] M. Pieri, S. Bhattacharya, I. Fisk, J. Letts, V. A. Litvine and J. G. Branson, "Inclusive search for the Higgs boson in the $\mathrm{H} \rightarrow$ gamma gamma channel," CERN-CMS-NOTE-2006-112.

[3] G. L. Bayatian et al. [CMS Collaboration], "CMS technical design report, volume II: Physics performance," J. Phys. G G 34, 995 (2007).

[4] G. L. Bayatian et al. [CMS Collaboration], "CMS physics: Technical design report," CERNLHCC-2006-001.

[5] CMS Collaboration, "Electromagnetic calorimeter commissioning and performance with $7 \mathrm{TeV}$ data ," CMS PAS EGM-10-003, 2010.

[6] CMS Analysis Note AN-2011/129 "Search for a Higgs boson decaying into two photons in proton-proton collisions recorded by the CMS detector at the LHC", Higgs to gammagamma working group, August 2011

[7] CMS Collaboration, "Search for a Higgs boson decaying into two photons in the CMS detector," CMS PAS HIG-11-010, 2011.

[8] CMS Collaboration, "Search for a Higgs boson decaying into two photons in the CMS detector," CMS PAS HIG-11-021, 2011.

[9] CMS Analysis Note AN-2011/426 "Search for a Higgs boson decaying into two photons in pp collisions recorded by the CMS detector at the LHC", Higgs to gammagamma working group, December 2011

[10] CMS Collaboration, "Search for a Higgs boson decaying into two photons in the CMS detector," CMS PAS HIG-11-030, 2011.

[11] S. Chatrchyan et al. [CMS Collaboration], "Search for the standard model Higgs boson decaying into two photons in pp collisions at sqrt(s)=7 TeV," Phys. Lett. B 710, 403 (2012) [arXiv:1202.1487 [hep-ex]].

[12] CMS Analysis Note AN-2012/048 "Search for a Standard Model Higgs boson decaying into two photons employing multivariate methods", Higgs to gammagamma working group, March 2012

[13] CMS Collaboration, "Higgs to Gamma Gamma, next generation (MVA)," CMS PAS HIG-12001, 2012.

[14] M. Pieri [CMS Collaboration], "Searches for the Standard Model Higgs Boson at CMS," arXiv:1205.2907 [hep-ex].

[15] CMS Collaboration, "Fermiophobic Higgs Search," CMS PAS HIG-12-002, 2012. 
[16] CMS Analysis Note AN-2012/035 "Searches for Fermiophobic Higgs Boson Decaying into Two Photons in the CMS Detector Using 2011 Data", Higgs to gammagamma working group, March 2012

[17] S. Chatrchyan et al. [CMS Collaboration], "Search for a fermiophobic Higgs boson in $p p$ collisions at $\sqrt{s}=7 \mathrm{TeV}, "$ arXiv:1207.1130 [hep-ex].

[18] CMS Analysis Note AN-2012/160 "Search for a Standard Model Higgs boson decaying into two photons in 2012 data", Higgs to gammagamma working group, June 2012

[19] CMS Collaboration, "Evidence for a new state decaying into two photons in the search for the standard model Higgs boson in pp collisions," CMS PAS HIG-12-015, 2012

[20] CMS Analysis Note AN-2012/213 "Search for fermiophobic Higgs boson in diphoton channel with 2012 data collected by the CMS", Higgs to gammagamma working group, June 2012

[21] CMS Collaboration, "Search for the fermiophobic model Higgs boson decaying into two photons in pp collisions at sqrt(s) $=7$ and $8 \mathrm{TeV}$," CMS PAS HIG-12-022, 2012

[22] CMS Analysis Note AN-2010/038 "Electron GSF Tracking Commissioning with first LHC Data ", M. Sani, M. Pieri, J. Branson, April 2010.

[23] CMS Analysis Note AN-2010/235 "Electron Commissioning in CMS from first 7 TeV Minimum Bias Data", S. Baffioniet al., November 2010

[24] CMS Collaboration, "Electron reconstruction and identification at sqrt(s) $=7$ TeV," CMS PAS EGM-10-004, 2010.

[25] CMS Analysis Note AN-2009/178 "Electron Identification in CMS", S. Baffioni et al., December 2009 .

[26] CMS Collaboration, "Electromagnetic calorimeter commissioning and performance with $7 \mathrm{TeV}$ data," CMS PAS EGM-10-002, 2010.

[27] CMS Collaboration, "Photon reconstruction and identification at sqrt(s) = 7 TeV," CMS PAS EGM-10-005, 2010.

[28] CMS Collaboration, "Isolated Photon Reconstruction and Identification at sqrts=7 TeV," CMS PAS EGM-10-006, 2010.

[29] CMS Analysis Note AN-2010/268 "Measurement of isolated photon production cross section in pp collisions at $\mathrm{s}=7 \mathrm{TeV}$ ", S. Ganjour et al., March 2011

[30] CMS Analysis Note AN-2010/320 "Search for an Excited Electron in pp Collisions at sqrt(s) $=7$ TeV", S. Bhattacharya, S. Jain, M. Pieri, E. Sudano and Y. Yang, March 2011

[31] S. Chatrchyan et al. [CMS Collaboration], "A search for excited leptons in pp Collisions at sqrt(s) = 7 TeV," Phys. Lett. B 704, 143 (2011) [arXiv:1107.1773 [hep-ex]].

[32] M. Pieri, G. Maron, A. Brett, E. Cano, S. Cittolin, S. Erhan, D. Gigi and F. Glege et al., "CMS DAQ event builder based on gigabit ethernet," FERMILAB-CONF-06-449-E.

[33] G. Bauer, V. Boyer, J. Branson, A. Brett, E. Cano, A. Carboni, M. Ciganek and S. Cittolin et al., "CMS DAQ event builder based on Gigabit Ethernet," IEEE Trans. Nucl. Sci. 55, 198 (2008). 
[34] G. Bauer, B. Beccati, U. Behrens, K. Biery, A. Brett, J. Branson, E. Cano and H. Cheung et al., "The CMS event builder and storage system," J. Phys. Conf. Ser. 219, 022038 (2010).

[35] V. Khachatryan et al. [CMS Collaboration], "Observation of Long-Range Near-Side Angular Correlations in Proton-Proton Collisions at the LHC," JHEP 1009, 091 (2010) [arXiv:1009.4122 [hep-ex]].

[36] S. Chatrchyan et al. [CMS Collaboration], "Search for $B_{s}^{0} t o \mu^{+} \mu^{-}$and $B^{0}$ to $\mu^{+} \mu^{-}$decays," JHEP 1204, 033 (2012) [arXiv:1203.3976 [hep-ex]].

[37] S. Chatrchyan et al. [CMS Collaboration], "Search for B(s) and B to dimuon decays in pp collisions at 7 TeV," Phys. Rev. Lett. 107, 191802 (2011) [arXiv:1107.5834 [hep-ex]].

G Bauer et al.

[38] G. Bauer et al. "Upgrade of the CMS Event Builder," CMS CR-2012/172 (2012)

[39] “Observation of WZ Production”, PRL 98 (2007) 161801.

[40] "Strong Evidence for ZZ Production in $p \bar{p}$ Collisions at $\sqrt{s}=1.96$ TeV", PRL 100 (2008) 201801.

[41] "A Study of WW, ZZ and Higgs Production in Dilepton plus Missing Transverse Energy Final State at CDF Run II", Shih-Chieh Hsu, UCSD Thesis 2008.

[42] "Search for Higgs Boson Decaying to Two W Bosons at CDF", PRL 102 (2009) 021802.

[43] "The Pilot Way to Grid Resources Using glideinWMS", doi:10.1109/CSIE.2009.950 (2009).

[44] "First Measurement of the Cross Section for Top-Quark Pair Production in Proton-Proton Collisions at at $\sqrt{s}=7 \mathrm{TeV} "$, Phys.Lett.B 695 (2011) 424-443.

[45] "Measurement of the ttbar Production Cross Section and the Top Quark Mass in the Dilepton Channel in pp Collisions at $\sqrt{s}=7$ TeV", JHEP 07 (2011) 049.

[46] "Measurement of $W^{+} W^{-}$Production and Search For the Higgs Boson in pp Collisions at $\sqrt{s}=7$ TeV", Phys. Lett. B 699 (2011) 25.

[47] "Search For Physics Beyond the Standard Model in Opposite-Sign Dilepton Events at $\sqrt{s}=7$ TeV", JHEP 1106 (2011) 026.

[48] "Search for New Physics with Same-Sign Isolated Dilepton Events with Jets and Missing Transverse Energy at The LHC", JHEP 1106 (2011) 077.

[49] "Search for Same-Sign Top-Quark Pair Production at sqrt(s) $=7 \mathrm{TeV}$ and Limits on Flavour Changing Neutral Currents in the Top Sector", JHEP 1108 (2011) 005.

[50] "Search for the Standard Model Higgs Boson Decaying to a W pair in the Fully Leptonic Final State in pp Collisions at $\sqrt{s}=7$ TeV", Phys.Lett.B 710 (2012) 91-113.

[51] "Observation of a new boson at a mass of $125 \mathrm{GeV}$ with the CMS experiment at the LHC", arXiv.org/abs/arXiv:1207.7235

[52] "Search for the Standard Model Higgs Boson in the H to ZZ to 21 2nu Channel in pp Collisions at $\sqrt{s}=7 \mathrm{TeV} "$, JHEP $1203(2012) 040$. 
[53] "Measurement of the WW Production Cross-section with the Full 2011 Dataset", CMS AN2012/061, in internal CMS review.

[54] "Search for Heavy Top-like Quark Pair Production in the Dilepton Final State in pp Collisions at $\sqrt{s}=7 \mathrm{TeV}$ ", http://arxiv.org/abs/1203.5410

[55] "Search for New Physics with Opposite-sign Leptons, Jets, and Missing Transverse Energy in pp Collisions at $\sqrt{s}=7 \mathrm{TeV}$ ", http://arXiv.org/abs/arXiv:1206.3949.

[56] "Search for Physics Beyond the Standard Model in Events with a Z boson, Jets, and Missing Transverse Energy in pp Collisions at $\sqrt{s}=7 \mathrm{TeV}$ ", http://arXiv.org/abs/arXiv:1204.3774.

[57] "Search for New Physics with Same-sign Isolated Dilepton Events with Jets and Missing Transverse Energy in pp Collisions at $\sqrt{s}=7 \mathrm{TeV} "$, http://arXiv.org/abs/arXiv:1205.6615.

[58] "Search for New Physics in Events with Same-Sign Dileptons and B-tagged Jets in pp Collisions at $\sqrt{s}=7 \mathrm{TeV} "$, http://arXiv.org/abs/arXiv:1205.3933.

[59] S. Dimopoulos and G. F. Giudice, "Naturalness Constraints in Supersymmetric Theories with Nonuniversal Soft Terms," Phys.Lett.B 357, 573 (1995)

[60] M. Papucci, J. T. Ruderman and A. Weiler, "Natural SUSY Endures," http://arxiv.org/abs/1110.6926.

[61] "Search for Supersymmetry in Events with Same-sign Dileptons", CMS PAS SUS-12-017.

[62] "Search for Direct EWK Production of SUSY Particles in Trilepton Final States ", CMS AN-2012/051, in internal CMS review.

[63] "Measurement of the Top Polarization in the Dilepton Final State", CMS PAS TOP-12-016.

[64] "Top Charge Asymmetry Measurement in Dilepton Final State", CMS AN-2012/190, in internal CMS review.

[65] "Measurement of Spin Correlations in ttbar Production", CMS PAS TOP-12-004.

[66] "Measurement of WW production rate at 7TeV", CMS PAS SMP-12-005.

[67] "Measurement of WW production rate at 8TeV", CMS PAS SMP-12-013. 


\title{
Task T1:
}

\section{Theoretical Particle Physics}

\author{
University of California, San Diego
}

This Final Report for budget period July 1, 2010 - April 30, 2013 is submitted to the Department of Energy by the Regents of the University of California

August 4, 2013

Kim Griest

Benjamín Grinstein

Ken Intriligator

Julius Kuti kgriest@ucsd.edu

bgrinstein@ucsd.edu

keni@ucsd.edu

jkuti@ucsd.edu 


\section{Contents}

1 Final Report: Research 1

Final Report: Research 1

1 Griest ................................ 1

2 Grinstein . . . . . . . . . . . . . . . . . . 5

3 Intriligator . . . . . . . . . . . . . . . . . . . 11

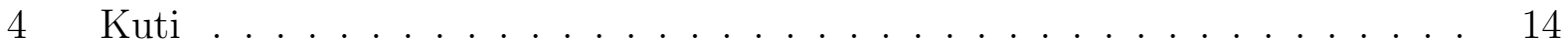

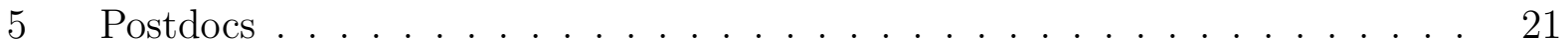

2 Contract Publications $\quad 26$

3 People $\quad 27$ 


\section{Final Report: Research}

\section{Griest}

\section{$1.1 \quad$ Overview}

(Kim Griest with students Agnieszka Cieplak and Joseph Fedrow)

This past year Griest and his group worked in two different areas. First, with student Agnieszka Cieplak, who is graduating and taking a postdoctoral position at Brookhaven National Lab, he worked on new limits on Primordial Black Hole (PBH) Dark Matter (DM). Second, with student Joseph Fedrow, he worked on anti-anthropic solutions to the cosmic coincidence problem.

\subsection{Primordial Black Hole Dark Matter}

Griest and Cieplak finished a detailed theoretical study and are nearing completion of a search through the Kepler satellite data for microlensing signatures of Primordial Black Hole (PBH) Dark Matter (DM). Griest and collaborators first suggested this as a method of searching for PBH DM in 2011 [100] and an improved estimate including stellar limbdarkening and better characterization of the Kepler source stars was published this year[41]. We then used our newly developed techniques to actually search through two years of the publicaly available Kepler lightcurve data. We have not found any good microlensing candidates, so can set a limit on the density of PBH DM in a new mass range. Our preliminary results rule out an additional order of magnitude of PBH DM parameters space, finding that PBHs with masses between $2 \times 10^{-9}$ and $10^{-7}$ cannot make up the entirety of the DM.

\subsection{Anti-Anthropic Solutions to the Cosmic Coincidence Problem}

The discovery of the Dark Energy (DE) exacerbates two fine-tuning problems. First, the cosmological constant problem: if the DE is a cosmological constant, its value is a hundred orders of magnitude smaller than typical particle physics numbers. Second, the cosmic coincidence problem: while the density of matter and energy drop rapidly as the Universe expands, vacuum energy (aka DE) does not. If the value of the DE density was larger than the measured value then it would have dominated the Universe long ago, stopping formation of galaxies, stars, and intelligent life. If the value was much small than measured, we wouldn't know it existed. In previous work [88], Griest showed that an ensemble of scalar fields could solve the cosmic coincidence problem without an invocation of any anthropic type argument. The basic idea is that each scalar fields has a time when it's density is significant, it causes a change in the cosmic equation of state for a short while, and then fades away. Thus in this model the current accelerated expansion is only a temporary situation. A major problem of this model was that it required extreme fine-tuning of the ensemble of scalar fields, thus solving one fine-tuning problem by introducing another. The past year, Griest and Fedrow have explored and found classes of scalar fields that exhibit tracking behavior and which do not need to be finely-tuned. This work is in progress but should make the anti-anthropic solution to the cosmic coincidence problem more palatable. 


\subsection{Previous Work}

Over the course of this grant Griest and his group have worked in many areas of particle astrophysics, but his main contributions have been in the areas of dark matter and dark energy. Griest likes to stay close to the experimental world, and his publications have invented or developed several new experimental techniques for the search for dark matter and other topics in particle astrophysics. Some of these past works are discussed below.

It is now well established that ordinary matter made of atoms contributes less than $5 \%$ of the energy density of the Universe, with the bulk consisting of Dark Energy (DE) (around $70 \%$ ) and Dark Matter (DM) (around 25\%). We know that dark energy and dark matter exist due to their gravitational influence but have no non-gravitational detection of either of these dominant substances, and thus have little guidance as to their fundamental nature. Discovering the nature of these "substances" is one of the main goals of modern physics and the main thrust of Griest's work.

Since there is so little little experimental guidance as to the nature of the dark matter, one can only proceed by hypothesizing a candidate and then thinking of experiments that might be able to detect or rule that candidate out. There have been hundreds of such candidates proposed, mostly from models of particle physics beyond the standard model. Griest wrote some of the first and most influential papers on the most popular of these candidates: the neutralino from supersymmetry (SUSY). For example, his discovery of the coherent neutralino-nucleus scalar interaction for spin-independent cross sections [85] (260 citations) ${ }^{1}$ has been the basis for all subsequent calculations of neutralino-nucleus elastic scattering, the main cross section needed for limits from direct detection experiments. Griest wrote a number of other influential papers in this subject, on both direct and indirect detection methods, for example, [84] (190 cites), [95] (180 cites), [123] (79 cites), culminating in a Physics Reports on Supersymmetric Dark Matter [122] that has received more than 2000 citations. One of the main motivations for supersymmetric DM was the WIMP "miracle" that typical SUSY parameter values gave relic abundances of neutralinos near the needed experimental value. In [98] Griest and Seckel wrote an important paper for the field of relic abundance calculation. He invented the concept and term "co-annihilation" where the calculated relic abundance of a DM particle from the Early Universe can be drastically modified due the presence of other, non-dark matter, particles. This paper has received more than 500 citations, but the term, concept, and methods of co-annihilation have entered the field so thoroughly that they are usually used now without attribution to Griest's original paper. Other influential papers were written on more general particle dark matter candidates, including some of the first work on non-topological solitons [96] and unitary limits on the mass of any dark matter particle [94] (200 cites).

Besides particle dark matter candidates beyond the standard model, Griest has also made major contributions to other dark matter candidates, especially Massive Compact Halo Objects (MACHOs). The basic idea [128] was that if dark matter consisted of black holes, dead stars, planets, non-topological solitons, etc. they could act as gravitational lenses and be detected by the magnification they caused to ordinary stars as they passed in front of

\footnotetext{
${ }^{1}$ The citation counts given here and below are from either Inspire or the Smithsonian/NASA ADS server. Inspire misses many citations when they appear in more astronomical journals, while ADS misses citations from some particle physics sources. For example, Griest's h-index is given as 51 by ADS and 36 by Inspire.
} 
them. In an influential paper [87] (270 cites), Griest coined the term MACHO and worked out in detail how an experiment could use this gravitational microlensing effect to detect or limit MACHO dark matter.

He also wrote a theoretical paper [86] which was the first (simultaneous with a Paczynzki paper) to suggest that by monitoring stars in the Galactic Bulge one could detect stars gravitationally lensing other stars. The method Griest suggested is now the source of the huge numbers of microlensing events that astrophysicists use for many purposes today. This method is also one of the main methods to be used by NASA's WFIRST project, which was the top ranked project of the "New Worlds, New Horizons in Astronomy and Astrophysics," the 2010 Decadal Review.

Griest then joined one of the experimental collaborations, the MACHO collaboration led by Charles Alcock, and led the analysis team. Thus he was part of the first detection of gravitational microlensing [9] (620 cites). Griest and his students led the analysis of this very successful experiment which ruled out MACHOs as the primary constituent of the DM, and eliminated objects with masses between $2 \times 10^{-8} \mathrm{M}_{\odot}$ and $30 \mathrm{M}_{\odot}$ as the primary constituent of the dark matter. [10, 11] (500 cites). The MACHO collaboration elimination of 8 orders of magnitude of parameter space stands still today as one of the most powerful experimental constraints ever found on dark matter. Griest also did other theoretical work on gravitational lensing that helped turn it into the powerful tool it is today, for example, [97] (150 cites), and [93](80 cites).

Griest has also had a long standing interest in Dark Energy (DE) [26]. Griest was very involved with SNAP and then JDEM, serving as co-chair of the JDEM science definition team [83]. As mentioned above, DE is known to make up around $70 \%$ of the energy density of the Universe, but the only detection of this "substance" comes from model fits of the expansion history of the Universe. The expansion is measured to be accelerating, implying that DE must have a negative equation of state parameter, $w$, and there are several very different theories proposed to accomplish this. The important scientific goal is to distinguish between three main possibilities: DE is a cosmological constant so $w=-1$ exactly, DE is the effect of a "rolling scalar field" (quintessence) so $w \neq-1$ and $d w / d t \neq 0$, or DE is an effect of modifications to General Relativity.

While continuing his interest in Dark Matter, and continuing to give invited review talks on the theory of particle dark matter and dark energy (e.g. most recently at the HiPACC Computational Astronomy conference in Santa Cruz, 2012, the Focus Week on Dark Matter at IPMU, Tokoyo, 2011, and the Ninth Symposium on Sources of Dark Matter and Dark Energy, UCLA, 2010).

Griest also has worked in other areas of particle astrophysics. For example, it has been claimed that the fine structure constant (alpha) was smaller in the past than it is now [127]. If true this it would be a revolutionary shift in our understanding of the fundamental constants of nature. In modern theories it is possible that these constants are, in effect, vacuum expectation values of some fields, thus such a result could show that these models are true. Griest and his students made an important contribution to this field by developing a new technique for calibrating spectra of high redshift quasars. By analyzing data from the Keck Telescope HIRES spectrograph taken through the Keck iodine cell, Griest and his student showed that the Keck HIRES spectrograph was not calibratible to the precision previous workers had assumed [99] (47 cites). This was somewhat of a bombshell in the field 
since it called into question many previous results. Griest and his student then repeated their analysis on iodine cell data from the main competing telescope (UVES on the VLT), again finding much larger than suspected calibration errors [142]. While it is possible to average away these calibration errors with sufficient data, according to a leader in this field, these results are now widely accepted and called the "Griest" effect.

Griest has done substantial service for the DoE. For example, he severed as co-chair, with Chuck Bennett, of the Science Definition Team for the NASA/DoE Joint Dark Energy Survey (JDEM). This committee was tasked with defining the dark energy science and techniques that a satellite mission would perform. This mission was eventually dropped by the DoE, but continues as part of NASA's WFIRST mission. Griest also served as a DoE representative and eventually as chair of the Astronomy and Astrophysics Advisory Committee (AAAC). This congressionally mandated FACA committee issues a yearly report [89] and advises the DoE, NASA, NSF, Congress, and the White House on the status of Astronomy and Astrophysics in the U.S.. Griest has served on a number of DoE panels, including the SAGENAP subpanel of HEPAP, the Dark Energy Panel, the SLAC annual review board, Early Career Research Program Panel, the SNAP review board, and many grant review boards including for U Penn, Brookhaven National Laboratory, and Stanford Linear Accelerator Center.

Note also that Griest has been active in recruitment, mentoring, and implementing of policies in aid of diversity and underrepresented groups at UCSD. He has mentored several such students, most recently in Summer 2012, and one of his students won a national prize for their joint research. 


\section{Grinstein}

(Fortin, Stergiou, Pirtskhalava, Uttayarat, Murphy)

\section{Scale without conformal invariance}

Phases of Quantum Field Theory (QFT) are characterized by the long distance behavior (the IR limit) of correlation functions: either there is a mass gap in which case all correlation functions are exponentially suppressed and the model is IR-free, or there are massless particles in which case the correlation functions are power laws. In the latter case the model may still be IR-free (as in, for example, QED), but the more interesting case is that it may be interacting. It has been standard lore, and a theorem in two space-time dimensions, $D=2$ $[133,145]$, that all such interacting-IR limits correspond to Conformal Field Theories (CFT). The point is that they must be scale invariant, and it was believed (and proved in $D=2$ ) that sensible, interacting models that are scale invariant are necessarily also conformal (invariant under special conformal transformations). The question remained, does scale imply conformal invariance in $D=4$ ?

There is another way to examine the IR-behavior of QFT models, and that is by finding the IR-limit of renormalization group (RG) flows of the coupling constants of the model. Wilson classified the IR-limits of RG flows that could give rise to non-trivial interacting models [143]: one can have either (i) fixed points, (ii) limit cycles or (iii) ergodic flows. Common lore held that QFT models can only exhibit fixed points. This nicely matches the common lore that the only interacting-IR models are CFTs, since these correspond to the limiting behavior of RG flows with IR-fixed points.

These questions, namely the impossibility of scale but not conformal models and the possibility of limit cycles or limit ergodic flows, were solved recently. Grinstein, with postdoc Fortin and student Stergiou discovered models that display limit cycles or limit ergodic flows in relativistic QFT in $D=4$. They also proved perturbatively that unitary, renormalizable, relativistic scale invariant QFTs are necesarily conformal.

They studied RG flows of classically scale invariant models of $n_{s}$ real scalars and $n_{f}$ Weyl fermions possibly charged under a non-abelian gauge group. They first discovered models, without gauge interactions, in $D=4-\epsilon$ (asymptotically free even without gauge fields), whose two-loop RG flows exhibit limit cycles [69, 72]. They gave a proof, to all orders in perturbation theory, that for $n_{s} \leq 1$ and any $n_{f}$ there are no recursive RG flows. Moreover, they developed a systematic algorithm for the search of scale-invariant trajectories in perturbation theory [72] and established the scheme independence of the limit cycles and their stability properties. They later solidified the results by establishing they persist at three-loops, which is crucial since the rate of circulation along the cycle is formally of threeloop order [70]. The procedure was then extended to the more complex case of $D=4$ where non-abelian gauge fields must be included to insure asymptotic freedom [71].

These discoveries invalidate the gradient flow hypothesis, that in any given model the $\beta$-functions are given as the gradient in coupling constant space of a "potential" [141]. However there is no conflict or contradiction with the $a$-theorem. Using results of Jack and Osborn [121] on the restrictions that Weyl invariance imposes on the flow of $a$, Grinstein and collaborators showed that $a$ is constant precisely on limit cycles (or limit ergodic trajectories) [73] and showed perturbatively the strong version of the $a$-theorem, that $a$ decreases monotonically along an RG-flow (towards the IR). Moreover they computed explicitly and for the 
first time the leading (three loops) non-vanishing contribution to the Lie-algebra valued function $S$ of Jack and Osborn that generates field rotations along flows. This remarkable result allows determination of existence of flows given the zeroes of the beta functions computed at leading order (two loops for gauge couplings).

They went on to show that in $D=4$ scale inavarance implies conformal invriance. They first showed that on limit cycles or fixed points $S$ equals the generator of translations along the cycle (and vansihes on fixed points). This was then used to show that the trace of the stress energy tensor vanishes (up to equations of motion) on limit cycles, which was the remaining stumbling block in Polchinski's original argument. Finally, in collaboration with student Murphy, they showed there are no limit cycles in supersymmetric theories [77]. These results hang on the assumption of a positive definite metric in theory space, an assumption that is known to be true perturbatively.

\section{$\mathrm{p} \overline{\mathbf{p}}$ and pp collisions at high energy: new resonances and anomalies}

Recent observations [3,5] by the $\mathrm{CDF}$ and $\mathrm{D} \varnothing$ collaborations point to a forwardbackward asymmetry in $t \bar{t}$ production $\left(A_{F B}^{t \bar{t}}\right)$, significantly in excess of the SM prediction. New Physics (NP) models that explain these anomalies generally have new particles exchanged in the $s$ - or $t$-channels, and each case faces a number of challenges. For models with $s$-channel exchange, there is no evidence for a resonance in the $t \bar{t}$ invariant mass spectrum, pushing the mass of the particle to at least $\mathcal{O}(1 \mathrm{TeV})$. Further, in order to obtain a positive $\mathrm{A}_{\mathrm{FB}}^{\mathrm{t} \overline{\mathrm{t}}}$, the new exchanged particle has to couple to light quarks with an opposite sign than to the top. These couplings can lead to large flavour changing neutral currents (FCNC's). Models with $t$-channel exchange require large inter-generational couplings. It is challenging for such scenarios to be incorporated into a realistic model of flavour. In concrete models, nonzero $\bar{c} u$ couplings can lead to unacceptably large $\mathrm{D}$ meson mixing. $t$-channel exchanges can also lead to same sign top-pair production, which is tightly constrained [2].

Grinstein and collaborators Trott (CERN) and Kagan and Zupan (Cincinnati) demonstrated that all of the above obstacles, as well as dijet constraints, can be overcome, if the NP particle exchanged in the $t$ or $s$ channel is in a nontrivial representation of the flavor group $\mathrm{G}_{\mathrm{F}}[112,113]$. For instance, an $s$-channel exchange of an $\mathrm{SU}(3)_{\mathrm{U}}$ octet vector field automatically has couplings to light quarks of opposite sign than to the top quark $\frac{1}{\sqrt{3}} V_{\mu}^{8}\left(\bar{u}_{R} \gamma^{\mu} u_{R}+\bar{c}_{R} \gamma^{\mu} c_{R}-2 \bar{t}_{R} \gamma^{\mu} t_{R}\right)+\cdots$, where the ellipses denote the remaining field components of the 8 representation. The same flavour octet vector will also lead to $u \leftrightarrow t$ transitions in the $t$-channel $\left(\bar{U}_{R} T^{A} \gamma^{\mu} U_{R}\right) V_{\mu}^{A}=\left(V_{\mu}^{4}-i V_{\mu}^{5}\right)\left(\bar{t}_{R} \gamma^{\mu} u_{R}\right)+\cdots$, from the exchange of $V_{\mu}^{4,5}$ octet components, and similarly to $c \leftrightarrow t$ transitions from the exchange of $V_{\mu}^{6,7}$. Despite the large inter generational transitions, no FCNC's arise before flavour breaking. For $\mathrm{G}_{\mathrm{F}}$ breaking they assume Minimal Flavour Violation (MFV), where the flavour violation (FV) in the NP sector is proportional to Yukawa couplings of the standard model. As a result FCNC's are consistent with experiment even for NP mass scales $<\mathrm{TeV}$. With student Murphy and collaborator Trott, Grinstein studied further the constraints that electroweak precision tests impose on these models [105].

Grinstein and students Murphy and Uttayarat and postdoc Pirtskhalava showed that the anomalously large top quark forward-backward asymmetry observed by CDF and DØ can naturally be accommodated in models with flavor-violating couplings of a new massive spin-2 state to quarks [114]. The lowest-order couplings of a spin-2 filed to fermions are analogous to the those of a graviton, leading to strong sensitivity in spin-2 mediated amplitudes to the 
transferred energy. Hence the observed dependence of the asymmetry on the $t \bar{t}$ invariant mass fits nicely into the proposed framework. In particular, they find a vast parameter space which can lead to the central value for the observed forward-backward asymmetry in the high mass bin, while being in accord with all of the existing experimental constraints.

Grinstein and student Murphy computed the bottom quark forward-backward asymmetry at the Tevatron in the Standard Model and for several new physics scenarios [104]. Near the $Z$-pole, the SM bottom asymmetry is dominated by tree level exchanges of electroweak gauge bosons. While above the $Z$-pole, next-to-leading order QCD dominates the SM asymmetry as was the case with the top quark forward-backward asymmetry. Light new physics, $M_{N P} \lesssim$ $150 \mathrm{GeV}$, can cause significant deviations from the SM prediction for the bottom asymmetry. The bottom asymmetry can be used to distinguish between competing NP explanations of the top asymmetry based on how the NP interferes with $s$-channel gluon and $Z$ exchange.

Grinstein has had a long term interest in properties of the higgs boson [39, 101, 109, 110]. More recently, he considered the signatures of a light dilaton in higgs-less models and the difficulties in distinguishing the dilaton from an elementary higgs [81]. This work rekindled an old debate on whether walking technicolor (WTC) may have a light dilaton in its spectrum $[21,115]$. To get some insight into this question, Grinstein and student Uttayarat presented in [111] a completely perturbative model that displays behavior similar to that of WTC. In one phase of the model RG-flows run towards an IR-fixed point but approximate scale invariance is spontaneously broken before reaching the fixed point. The trajectories then run away from it and a light dilaton appears in the spectrum. The mass of the dilaton is controlled by the "distance" of the theory to the critical surface, and can be adjusted to be arbitrarily small without turning off the interactions. There is a second phase with no spontaneous symmetry breaking, hence no dilaton, and in which RG trajectories do terminate at the IR-fixed point.

While there is a vast literature exploring the phenomenological effects of The Littlest Higgs model [23], the renormalization structure of the model has been little explored. Grinstein and students Uttayarat and Kelley have noted [102] that renormalization group running of the top Yukawa coupling in Littlest Higgs models disrupts the collective symmetry. That is, in order for the collective symmetry argument to operate in the top-quark Yukawa sector, the coupling is designed so as to satisfy an $S U(3)$ symmetry. However, this symmetry is broken by weak gauge interactions. The would be $S U(3)$ symmetric top-Yukawa actually splits into two $S U(2) \times U(1)$ symmetric terms with coupling constants that run away from each other as they evolve under the renormalization group. They went on to determine the full renormalization group structure of the Littlest Higgs model at one loop order [103]. They found that not only is the top-yukawa term split into collective symmetry breaking terms, but find a host of counterterms that break the collective symmetry as well. These counterterms are not included at tree level in the standard formulation but are not suppressed in the derivative expansion. They are excluded arbitrarily.

\section{Flavor physics and CP violation}

In Minimal Flavor Violation (MFV), as in the SM, the only source of flavor violation arises from the Yukawa couplings. In the limit of vanishing quark masses the SM Lagrangian has a large global flavor (or "horizontal") symmetry. Imposing this symmetry on beyond the SM (BSM) physics is effective in suppressing new sources of FCNC. Remarkably, the scale of such new physics can be as low as a few $\mathrm{TeV}$. This suggests a dynamical origin for 
the Yukawas, as spurions, that is VEVs of scalar fields carrying the same flavor quantum numbers as the mass terms of the SM. Spontaneous breaking of the flavor symmetry gives rise to unacceptable (flavor changing) massless Goldstone bosons. Grinstein and CERN postdocs Redi and Villadoro studied the possibility of gauging the Standard Model flavor group [108]. Anomaly cancellation requires additional fermions. All flavor violating effects turn out to be controlled roughly by the SM Yukawas, suppressing transitions for the light generations. Due to an automatic inverted hierarchy the scale of new gauge flavor bosons could be as low as the electroweak scale without violating any existing bounds but accessible at the Tevatron and the LHC. The mechanism of flavor protection potentially provides an alternative to Minimal Flavor Violation, with flavor violating effects suppressed by hierarchy of scales rather than couplings.

Grinstein and collaborators realized that the Higgs mass in higher derivative versions of the SM does not suffer from a quadratic divergence [51, 107]. Radiative corrections shift the Higgs mass by $\sim M / 4 \pi$, where $M$ is the scale characterizing the higher derivatives. Lee and Wick argued that this class of theories can be made consistent if the would-be negative metric states are unstable. This "Lee-Wick Standard Model" (LWSM) is consistent with electroweak precision data $[12,35,37,140]$ and with flavor physics constraints provided $M$ is at least a few TeV [49]. Such low values for $M$ have observable effects, resonances associated with would-be negative metric states, roughly one per SM particle.

The quantization procedure of Lee and Wick gives time advancement in certain scattering amplitudes. In the LWSM, with $M \sim$ few $\mathrm{TeV}$, these time advancements are unmeasurably short. But can a macroscopic sequence of non-causal effects produce macroscopic violations of cause and effect? Grinstein and collaborators[68] attempted to address this question indirectly, studying the behavior of LW models at high temperature. For a gas of bosons the speed of sound was found to increase with temperature but never to exceed the speed of light. However, somewhat surprisingly (and discouragingly) they discovered that the energy density of a LW gas of fermions decreases without bound as the temperature is increased.

Grinstein took on this question again with collaborator Espinoza. They investigated the ultraviolet properties of the effective potential, $V_{\text {eff }}$, of LW theories with scalars, fermions and gauge bosons [52]. There is an interesting connection between UV and finite temperature behavior. Symmetry non-restoration can occur in models for which quadratic divergences in the Higgs mass cancel among states with same statistics. Heuristically, this is because $T^{2} m^{2} / v^{2}$ corrections to the finite temperature effective potential, which are responsible for symmetry restoration, are directly related to quadratic divergences to the Higgs mass at zero temperature. Since in LW theories cancellation of divergences are among states with same statistics one expects that EW symmetry does not get restored at high temperature. However, it is not immediately obvious how to extend the standard calculation of the finite temperature effective potential to LW models. Nevertheless, if one ignores the subtleties of quantization of Lee-Wick theory and simply replace the energy integral in $V_{\text {eff }}$ by a sum of Matsubara modes, it is tantalizingly found that the LW modes enter the effective potential with normal signs, but still give the expected cancellation in the thermal $T^{2}$ shift of the mass. This is at odds with Grinstein's previous calculation of the free energy (directly from the $S$-matrix). It is still not know which one of these two results, if either, is correct.

As a by-product, from the effective potential they determine some renormalization group equations (RGEs) for scalar self couplings (Grinstein and O'Connell [106] had determined 
RGEs for Yang-Mills LW models with fermions and scalars, excluding scalar self-couplings and Chivukula et al [38] determined the scalar self-coupling RGE in the LW Abelian-Higgs model). They also clarify an apparent discrepancy between RGEs in different formulations of the LW theory. Finally, solving the RGEs, Grinstein and Espinoza find that the running of the scalar self-coupling coupling is better UV-behaved in the LWSM than in the SM: it does not get driven to negative values at high energy if the Higgs mass is low nor does it blow-up below the Planck mass if the Higgs mass is large. As a result, in the LWSM the lower stability bound and the perturbativity bound on the Higgs mass disappear. Nevertheless, the RG evolution of gauge couplings above the LW mass scale $M$ is also modified. They find a Landau pole for the $U(1)_{Y}$ gauge coupling at a scale $\Lambda^{\prime} \sim 10^{16} \mathrm{GeV}$ (for $M \sim 1 \mathrm{TeV}$ ).

Soon after Belle's discovery [40] (and BaBar's confirmation [25]) of the $X(3872)$ resonance it was realized that this particle could not have been identified as a standard charmonium excitation, despite decaying prominently into $J / \psi \rho$ [135]. The mass of the $X$ being so close to the $D^{0} \bar{D}^{* 0}$ threshold suggests that it can be an example of a hadron molecule characterized by an extremely small binding energy. CDF and D0 have confirmed the $X(3872)$ in proton-antiproton collisions [6,7]. It seems odd that a such a loosely bound molecule could be produced promptly (i.e. not from $B$ decay) in a high energy hadron collision environment. Grinstein and collaborators established an upper bound on the cross section [30-32]. The only inputs to the bound are the production cross section of $D^{0} \bar{D}^{* 0}$ pairs, obtained from Herwig and Pythia, and the assumption that the relative momenta in the molecular wavefunction has support only for $\Delta k<30 \mathrm{MeV}$. Their finding, that the cross section is at least 30 times smaller than the minimum production cross section one can extract from CDF data, casts heavy doubts on the molecular interpretation of the $X(3872)$.

However Artoisenet and Braaten argued that final state interactions can dramatically increase the support of the wavefunction by an order of magnitude [24]. Grinstein and collaborators responded by showing that the molecular hypothesis with this enhancement leads to a hypothetical $D_{s}^{0} \bar{D}_{s}^{* 0}$ molecule, the $X_{s}\left(1^{++}\right)$, with mass $M_{X_{s}}=4080 \mathrm{MeV}$ and production cross section at the Tevatron of $3 \mathrm{nb}$ [33]. No such state has been reported by CDF or D0. Moreover, they note the use of Migdal-Watson's theorem by Artoisenet and Braaten is in a regime where the theorem does not apply.

Recently the BaBar collaboration took issue with the widely accepted hypothesis that that the $X(3872)$ is a $1^{++}$resonance, raising the possibility of $2^{+}$quantum numbers [20]. Assuming the $2^{+}$hypothesis, the molecular interpretation of the $X(3872)$ is ruled out while the charmonium and tetraquark interpretations remain viable. Grinstein and collaborators have made a model independent study of the decay modes of the $X(3872)$ under the hypothesis that it is a $1^{++}$or a $2^{-+}$state [34]. They give a general parametrization of the transition matrix elements describing the known decays in terms of a set of strong coupling constants which they compute from experimentally available decay widths. They conrm the negative parity assignment for the $X$ is indeed favored, using BaBar data [20].

The $J / \psi(\rho, \omega) \rightarrow X_{1,2} \rightarrow D^{0} \bar{D}^{0 *}$ rates could be of relevance to the study of a classic background to the $J / \psi$ suppression signal in heavy ion collisions: the $J / \psi$ may be absorbed by a hot gas of light hadrons at a similar rate as it is supposed to occur in a phase of deconfined quarks and gluons. If this mechanism is effective below the Hagedorn temperature then the $J / \psi$ suppression signal is a less compelling indicator of a new phase of matter in heavy ion collisions. Grinstein and collaborators showed that only the $X\left(2^{-+}\right)$, but not the 
$X\left(1^{++}\right)$, would contribute effectively to the $J / \psi(\rho, \omega) \rightarrow D^{0} \bar{D}^{0 *}$ absorption [34]. 


\section{Intriligator}

(Ken Intriligator, and Agarwal, Amariti, Fortin, Song, and Stergiou)

Intriligator and collaborators, including the postdocs and graduate students under his supervision, analyzed aspects of non-perturbative quantum field theory, renormalization group flows, supersymmetric gauge theories, and string theory. The works developed new tools and methods in supersymmetric quantum field theory, which can also be applied to model building, to alleviate phenomenological challenges. The hep-th vis hep-ph mix is guided by the LHC's results or BSM hints.

Intriligator and his group wrote the following publications during the last three years of the grant $[8,13-19,74-76,118,119,126,129]$. As a sample of the research results, the abstracts of some of these works are as follows:

In [118], we comment on various aspects of the the dynamics of $3 \mathrm{~d} N=2$ Chern-Simons gauge theories and their possible phases. Depending on the matter content, real masses and FI parameters, there can be non-compact Higgs or Coulomb branches, compact Higgs or Coulomb branches, and isolated vacua. We compute the Witten index of the theories, and show that it does not change when the system undergoes a phase transition. We study aspects of monopole operators and solitons in these theories, and clarify subtleties in the soliton collective coordinate quantization. We show that solitons are compatible with a mirror symmetry exchange of Higgs and Coulomb branches, with BPS solitons on one branch related to the modulus of the other. Among other results, we show how to derive Aharony duality from Giveon-Kutasov duality.

In [16], we explore some curiosities in $4 \mathrm{~d}$ susy RG flows. One issue is that the compelling candidate a-function, from a-maximization with Lagrange multipliers, has a 'strange branch,' with reversed RG flow properties, monotonically increasing instead of decreasing. The branch flip to the strange branch occurs where a double-trace deformation $\Delta W=O^{2}$ passes through marginality, reminiscent of the condition for the chiral symmetry breaking, out of the conformal window transition in non-susy gauge theories. The second issue arises from Higgsing vevs for IR-free fields, which sometimes superficially violate the a-theorem. The resolution is that some vevs trigger marginal or irrelevant interactions, leading to $\Delta a=0$ and decoupled dilaton on a subspace of the moduli space of vacua. This is contrary to classical intuition about Higgsing. This phenomenon often (but not always) correlates with negative R-charge for the Higgsing chiral operator.

In [119], we consider topology changing transitions for M-theory compactifications on Calabi-Yau fourfolds with background G-flux. The local geometry of the transition is generically a genus g curve of conifold singularities, which engineers a $3 \mathrm{~d}$ gauge theory with four supercharges, near the intersection of Coulomb and Higgs branches. We identify a set of canonical, minimal flux quanta which solve the local quantization condition on $G$ for a given geometry, including new solutions in which the flux is neither of horizontal nor vertical type. A local analysis of the flux superpotential shows that the potential has flat directions for a subset of these fluxes and the topologically different phases can be dynamically connected. For special geometries and background configurations, the local transitions extend to extremal transitions between global fourfold compactifications with flux. By a circle decompactification the M-theory analysis identifies consistent flux configurations in four-dimensional F-theory compactifications and flat directions in the deformation space of 
branes with bundles.

In [75], we consider using broken superconformal symmetry and the super operator product expansion (sOPE) to constrain and analyze hidden sector theories that couple to our gauge forces and are not necessarily weakly coupled. Conformal and supersymmetry breaking are IR effects, associated with field or spurion expectation values, whereas the sOPE is determined in the UV and hence does not notice the breaking. The broken superconformal symmetry relates OPE coefficients of superconformal descendant operators to those of the superconformal primaries. We apply these ideas to the current correlators of general gauge mediation (GGM). We also consider analyticity properties of these correlators, e.g. their discontinuities, and use the optical theorem to relate them to total scattering cross sections from visible to hidden sector states, e.g. $\sigma$ (vis + vis $\rightarrow$ hidden $)$, analogous to $\sigma\left(e^{+} e^{-} \rightarrow\right.$ hadrons) in QCD. We discuss how the current-current OPE can be truncated to the first few terms to get a good approximation to the visible sector soft masses of GGM.

In [74], we emphasize that, in superconformal theories, the OPE of superdescendants is generally undetermined from those of the superprimaries, and there is no universal notion of superconformal blocks. We recall these and related aspects of $4 \mathrm{~d}(\mathrm{~S}) \mathrm{CFTs}$, and then we focus on the super operator product expansion (sOPE) of conserved currents in $4 \mathrm{~d} N=1$ SCFTs. The current-current OPE J(x)J(0) has applications to general gauge mediation. We show how superconformal symmetry, when combined with current conservation, determines the OPE coefficients of superconformal descendants in terms of those of the superconformal primaries. We show that only integer-spin real superconformal primary operators of vanishing R-charge, and their descendants, appear in the sOPE. We also discuss superconformal blocks for four-point functions of the conserved currents.

In [76]: An often-exploited feature of the operator product expansion (OPE) is that it incorporates a splitting of ultraviolet and infrared physics. In this paper we use this feature of the OPE to perform simple, approximate computations of soft masses in gauge-mediated supersymmetry breaking. The approximation amounts to truncating the OPEs for hiddensector current-current operator products. Our method yields visible-sector superpartner spectra in terms of vacuum expectation values of a few hidden-sector IR elementary fields. We manage to obtain reasonable approximations to soft masses, even when the hidden sector is strongly coupled. We demonstrate our techniques in several examples, including a new framework where supersymmetry-breaking arises both from a hidden sector and dynamically.

In [129]: Recently it has been shown that the two-sphere partition function of a gauged linear sigma model of a Calabi-Yau manifold yields the exact quantum Kahler potential of the Kahler moduli space of that manifold. Since four-dimensional N=2 gauge theories can be engineered by non-compact Calabi-Yau threefolds, this implies that it is possible to obtain exact gauge theory Kahler potentials from two-sphere partition functions. In this paper, we demonstrate that the Seiberg-Witten Kahler potential can indeed be obtained as a two-sphere partition function. To be precise, we extract the quantum Kahler metric of 4D N=2 SU(2) Super-Yang-Mills theory by taking the field theory limit of the Kahler parameters of the $\mathrm{O}(-2,-2)$ bundle over $\mathrm{P} 1 \times \mathrm{P} 1$. We expect this method of computing the Kahler potential to generalize to other four-dimensional $\mathrm{N}=2$ gauge theories that can be geometrically engineered by toric Calabi-Yau threefolds. 


\section{Kuti}

(Julius Kuti and postdoc Chik Him Wong)

Research overview of last three years

Kuti and postdoc Wong worked in the last few years on the applications of non-perturbative lattice gauge theory methods to explore the theory space of four-dimensional strongly interacting gauge theories, an important subset of models with interesting ideas for new BSM physics. This subset, motivated by search for a new composite Higgs mechanism to replace the elementary scalar field, is actually a very large class of non-abelian gauge field theories with massless fermions in different realizations of three distinct phases of chiral and scale symmetries.

Model building requires the knowledge of the phase diagram as the number of colors $N_{c}$, number of fermion flavors $N_{f}$, and the fermion representation $R$ of the color gauge group (the new strong force) are varied. At fixed $N_{c}$ and $R$ and for small $N_{f}$ the theory is QCD-like in the chirally broken phase with asymptotic freedom from a negative $\beta$-function. If $N_{f}$ is large, the $\beta$ function is positive for all couplings, and the theory is trivial with a QED-like phase, with vanishing coupling when the cutoff is removed. There is some range of $N_{f}$ (conformal window) for which the $\beta$-function has a non-trivial zero, an infrared fixed point, where the theory is in fact conformal [27, 36]. This is reviewed with extensive references in [137]. Interesting BSM candidates require the theory to be very close to, but below the conformal window, with an almost zero $\beta$-function and a running coupling which is almost constant over a large energy range as schematically shown in Fig. 1.4. This is a critical issue in the discussion of scale symmetry breaking in association with the light pseudo-Goldstone dilaton state which could replace the light and elementary Higgs particle in the Standard Model with new physics on the composite $\mathrm{TeV}$ scale. Recently Kuti and collaborators

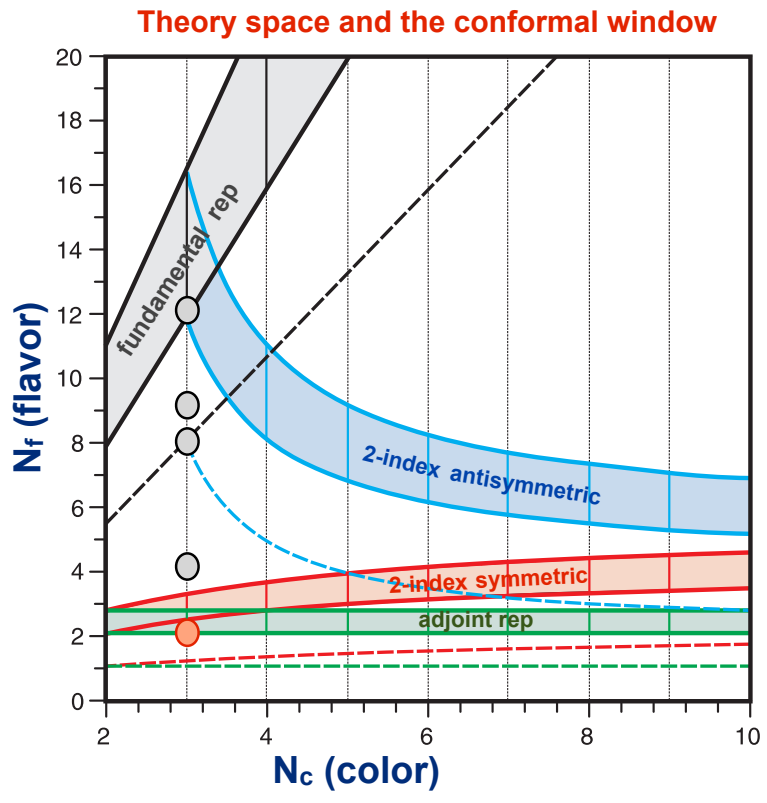

Fig. 1.1: The conformal window for $S U\left(N_{c}\right)$ gauge theories with $N_{f}$ massless fermions in various representations [48]. The shaded regions are the conformal windows, for fundamental (gray), 2-index antisymmetric (blue), 2-index symmetric (red) and adjoint (green) representations in Schwinger-Dyson approximation [137] which is being superseeded by lattice work at a rapid pace. The dashed lines, far off from what we know now, indicate earlier expectations for a larger conformal window which were based on the Banks-Zaks fixed point of two-loop $\beta$-functions. The color coded open circles mark the five gauge theories where Kuti and his collaborators studied the phase diagram and important spectral properties of the models. reported results from the sextet gauge model with two fermion flavors (marked with red circle in Fig. 1.1) as an important example of this dynamical scenario [61, 65].

Research results of Kuti and his collaborators during the DOE funded research period of the last three years include the study of the sextet gauge model showing important features 
for perhaps hiding the light Higgs impostor [61, 65], the invention and precise determination of a new running gauge coupling and its $\beta$-function from the Yang-Mills gradient flow in finite volume $[64,66]$, tests of chiral symmetry breaking and conformal scaling with two fermion flavors in the sextet representations of SU(3) color and twelve fermion flavors in the fundamental $\mathrm{SU}(3)$ color representation [59, 60, 63, 67], the phase diagram and chiral symmetry breaking with four, eight, and nine fermion flavors in the fundamental SU(3) color representation $[55,56,58]$, the strong confining force near the conform window and its connection with the running gauge coupling [53,62], the study of near-conformal chiral fermion properties using Random Matrix Theory [54], and gauge field topology in higher fermion representations [54, 57].

\section{The sextet gauge model and the Higgs impostor}

The stunning discovery of the $125 \mathrm{GeV}$ Higgs-like particle at the Large Hadron Collider does not exclude new BSM physics in the framework of some new strongly-interacting gauge theory with a composite Higgs mechanism, an idea which was outside experimental reach when it was first introduced as an attractive scenario. The original framework has been considerably extended by new explorations of the multi-dimensional theory space in fermion flavor number, the choice of color gauge group, and fermion representation. Even without spin and parity information, the new Higgs-like particle with decay modes not far from that of the Standard Model brings new focus and clarity to the search for the proper theoretical framework among the vast possibilities shown in Fig. 1.1.

An interesting example of this new focus is the light dilaton as a pseudo-Goldstone particle of spontaneous breaking of scale invariance that has been featured in recent phenomenological discussions as a conceivable interpretation of the discovery $[50,124]$. Nearly conformal gauge theories serve as theoretical laboratories for realistic implementations of this scenario $[21$, $28,81,111,115,116,144]$. Unfortunately, a credible realization of the idea as a strongly interacting BSM gauge theory is still lacking. Searching for a candidate theory, Kuti and collaborators investigated the two-index symmetric (sextet) representation of the $\mathrm{SU}(3)$ color gauge group with two fermion flavors $[61,65]$. They have shown that the model, marked with red circle in Fig. 1.1, satisfies two important and necessary conditions for perhaps hiding a light Higgs-like scalar state with or without dilaton-like interpretation. The model is close to the conformal window [46] and exhibits spontaneous chiral symmetry breaking $(\chi \mathrm{SB})$ with massless Goldstone bosons. The three massless Goldstone pions in the spectrum provide the minimal realization of the Higgs mechanism, just like in the original technicolor idea.

Simulations from Kuti and collaborators show that the sextet chiral condensate $\langle\bar{\psi} \psi\rangle$ is consistent with $\chi \mathrm{SB}$ and remains non-vanishing in the $m=0$ massless fermion limit. Two independent methods were used for the determination of the chiral condensate in the massless fermion limit. In the first method fits were made directly to $\langle\bar{\psi} \psi\rangle$ with constant and linear terms in the fitted function. For an independent determination, the subtracted chiral condensate operator was defined with the help of the connected chiral susceptibility $\chi_{\text {conn }}$. The two independent determinations give consistent non-vanishing fit results in the

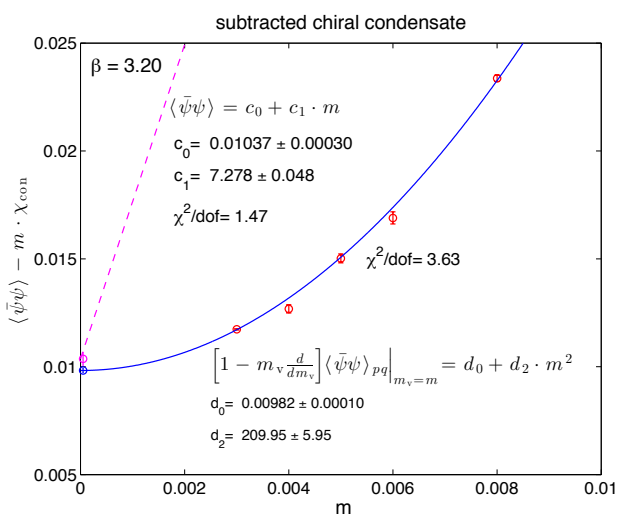

Fig. 1.2: The sextet chiral condensate with subtracted derivative is shown with $\chi \mathrm{SB}$ when extrapolated to $m=0$, consistent with direct fit to the condensate. 
massless chiral limit as shown in Fig. 1.2.

Additional strong evidence for the $\chi \mathrm{SB}$ phase was provided by the sextet spectrum. From the mass spectrum of the infinite volume extrapolations Kuti and collaboratos can probe the mass deformed conformal scaling behavior and compare with $\chi S B$ behavior when the fermion mass is varied in the infinite volume limit. From the tests they were able to perform, the sextet model is consistent with $\chi \mathrm{SB}$ and inconsistent with conformal symmetry.

The chiral Lagrangian describes the low energy theory of Goldstone pions and non-Goldstone pions in the staggered lattice fermion formulation. It is used as an effective tool probing the $\chi \mathrm{SB}$ hypothesis at finite fermion masses and in extrapolations to the massless chiral limit. Close to the chiral limit, the pion spectrum and the pion decay constant $F_{\pi}$ are organized in powers of the fermion mass $m$ which is an input parameter in the simulations. Chiral log corrections to the polynomial terms are generated from pion loops. Their analysis will require an extended dateset with high statistics as part of future plans. Based on the analytic fermion mass dependence of the chiral Lagrangian, and using the lowest four fermion masses, good polynomial fits were obtained without logarithmic loop corrections as shown in Fig. 1.3 for $M_{\pi}$ and $F_{\pi}$. The Goldstone nature of the pion state is clearly exhibited and $F_{\pi}$ in the $m=0$ limit is identified as the electroweak symmetry breaking scale.
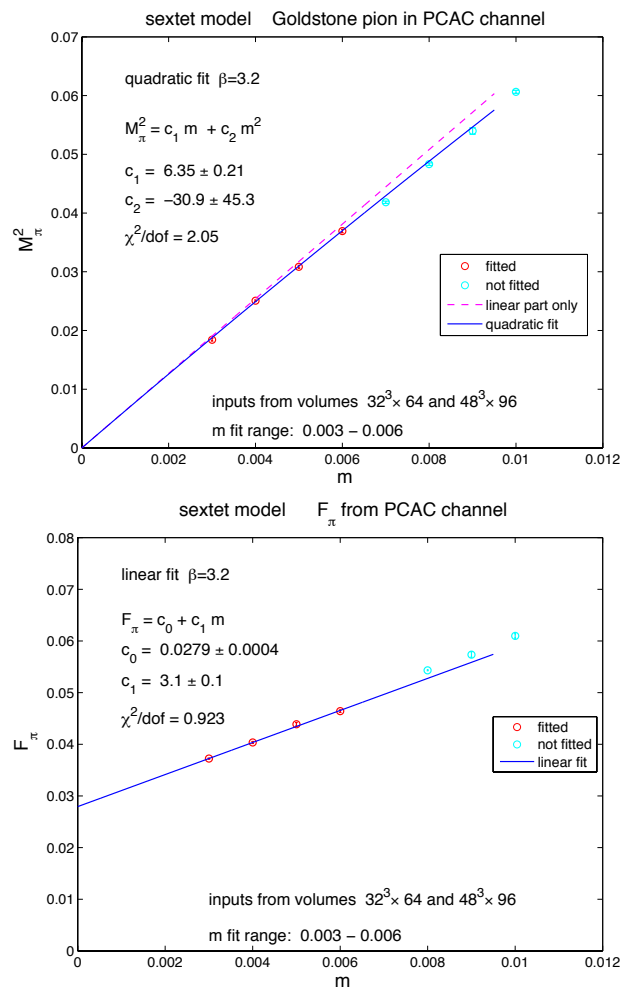

Fig. 1.3: Fits from the analytic mass dependence of the chiral Lagrangian without logarithmic loop corrections are shown for the Goldstone pion and $F_{\pi}$. The dashed line in the top plot for the Goldstone pion shows the leading linear contribution.

For comparison, under the conformal scaling hypothesis, the mass $M_{\pi}$ and the decay constant $F_{\pi}$ are given at leading order by $M_{\pi}=c_{\pi} \cdot m^{1 / 1+\gamma}$ and $F_{\pi}=c_{F} \cdot m^{1 / 1+\gamma}$. The coefficients $c_{\pi}$ and $c_{F}$ are channel specific but the exponent $\gamma$ must be universal in all channels. The leading scaling form sets in for small $m$ values, close to the critical surface. According to the hypothesis, there is an infrared conformal fixed point on the critical surface which controls the conformal scaling properties of small mass deformations. All masses of the spectrum can be subjected to similar conformal scaling tests, but Kuti and collaborators mostly focused on accurate data in the $M_{\pi}$ and $F_{\pi}$ channels. The incompatibility of the fitted $\gamma$ values in separate channels strongly disfavors the hypothesis, inconsistent with mass deformed conformal behavior [61, 65].

The consistency of all simulations, including [46], is resolved if the sextet model is close to the conformal window with a very small non-vanishing $\beta$-function. In this case the model exhibits the simplest composite Higgs mechanism and leaves open the possibility of a light scalar state with quantum numbers of the Higgs impostor emerging as the pseudoGoldstone dilaton state from spontaneous symmetry breaking of scale invariance. Even if scale symmetry breaking is entangled with $\chi \mathrm{SB}$ without dilaton interpretation, a light Higgs- 
like scalar state can emerge from new dynamics close to the conformal window. New research proposed by Kuti on the composite Higgs with lattice simulations in the sextet model may help to resolve these important problems.

\section{Running gauge coupling and the $\beta$-function from gradient flow of gauge fields}

It is very difficult to resolve the small and nearly vanishing $\beta$-function from the existence of a conformal fixed point gauge coupling. The main problem is to bridge many orders of magnitude in scale which is neccessary to run the gauge coupling from the UV fixed point toward the far infrared scale as schematically shown in Fig. 1.4. This cannot be done without unacceptable finite volume distortion in realistic lattice simulations. The first proposal to circumvent this difficulty turned the problem into the solution by running the gauge coupling with the finite size at renormalization scale $\mu=1 / L$ [125]. The Schrödinger functional of the method works well but requires Dirichlet boundary conditions on the fields which brings its own set of challenges. The most limiting one is the emergence of tunneling event in the simulations at stronger gauge couplings.

Kuti and collaborators recently proposed an innovative new gauge coupling $g(\mu=1 / L)$, running with the volume and defined from the gradient flow of the gauge field $[64,66]$. The new
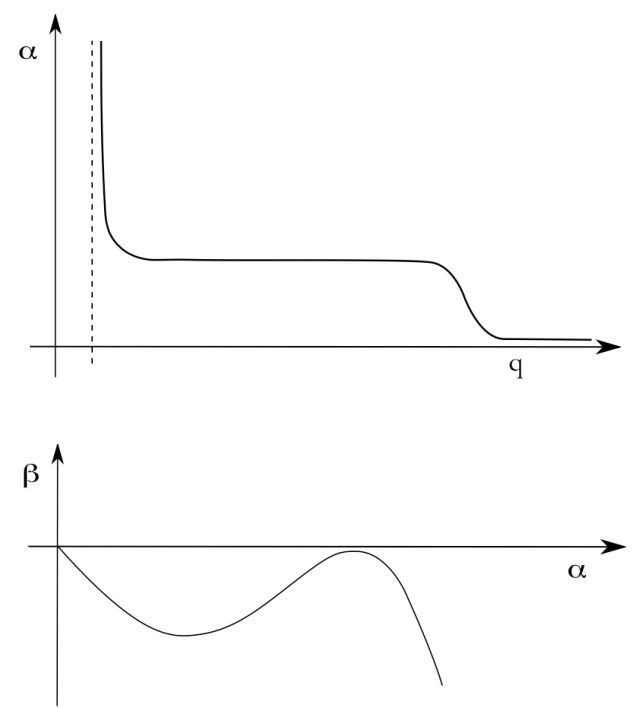

Fig. 1.4: The schematic top plot shows the walking-like behavior of the gauge coupling as the function of the momentum over a very large scale. The lower plot is the schematic form of the beta function associated with a generic walking theory and $\chi \mathrm{SB}$. running coupling scheme provides a very useful alternative to the Schrödinger functional scheme. Antiperiodic boundary conditions are used for massless fermion in all four directions of the finite four-dimensional volume and the boundary condition of the gauge field is kept periodic. The new running coupling scheme was tested with four massless fermions in the fundamental representation of the SU(3) color gauge group. It performs very well, with new plans for applications in BSM gauge theories. The measured renormalized couplings are very accurate, the scheme defines a one-parameter family which can be adjusted to several goals, and tunneling appears to be suppressed allowing to probe stronger renormalized gauge couplings.

Since the gradient flow probes the gauge field on the scale $\sqrt{8 t}$, where $\mathrm{t}$ is the flow time, a new running coupling can be defined as a function of $L$ in finite volume $V=L^{4}$ while holding $c=(8 t)^{1 / 2} / L$ fixed. This volume dependent coupling includes a normalization factor to match any definition of the renormalized coupling on the 1-loop level [66]. In tests with four massless fermions in the fundamental representation of the $\mathrm{SU}(3)$ color gauge group, $c=0.3$ was chosen probing the renormalized coupling on the scale $\mu=1 / 3 L$.

The fact that the volume is finite necessitates the separation of the gauge Fourier modes into zero and non-zero modes. The non-zero modes can be treated in 1-loop perturbation theory while the non-trivially interacting zero modes need to be treated exactly. The result at leading order contains both algebraically and exponentially suppressed finite volume 
correction terms relative to the infinite volume result.

\section{Finite size scaling analysis of conformal gauge theories}

Kuti and collaborators developed an important comprehensive test to probe conformal behavior for a strongly interacting gauge theory inside the conformal window [67]. The method, based on finite size scaling (FSS) theory, is completely general and some of its important ingredients have been well-known [47]. Kuti and collaborators tested conformal FSS with twelve fermions in the fundamenta representation of the $\mathrm{SU}(3)$ color gauge group. This model remains controversial sitting very close to the conformal window with difficulties in resolving conformal or $\chi \mathrm{SB}$ properties of the phase.

The expected leading FSS form for any mass $M$, or for $F_{\pi}$, scaled with the linear size $L$ of the spatial volume, is given by a scaling function $L \cdot M=f(x)$ where $x=L \cdot m^{1 / 1+\gamma}$ is the conformal scaling variable. The scaling form sets in close to the critical surface for small $m$ values. The scaling functions $f(x)$ can depend on the quantum numbers of the states but the scaling variable is expected to have the same form with identical $\gamma$ exponent in each quantum number channel. In sub-leading order there are conformal FSS scaling violation effects which are exhibited as a combined cutoff and $L$-dependent leading correction with the modified form $L \cdot M=$ $f(x)+L^{-\omega} g(x)$ where the scaling correction exponent $\omega$ is determined at the infrared fixed point (IRFP) $g^{*}$ of the $\beta$-function as $\omega=\beta^{\prime}\left(g^{*}\right)$. This assumes that the mass deformation away from the critical surface is the only relevant perturbation around the IRFP. The leading scaling correction term close enough to the critical surface dominates any other corrections which are supressed by further inverse powers of $L$. To detect the leading scaling violation effect requires high precision data with fits to scaling functions $f(x)$ and $g(x)$ and the critical exponent $\omega$. This is one of the objectives of the proposed research plan.

Conformal FSS theory was applied to the to the data sets of the controversial fermion model with twelve flavors in the fundamental SU(3)
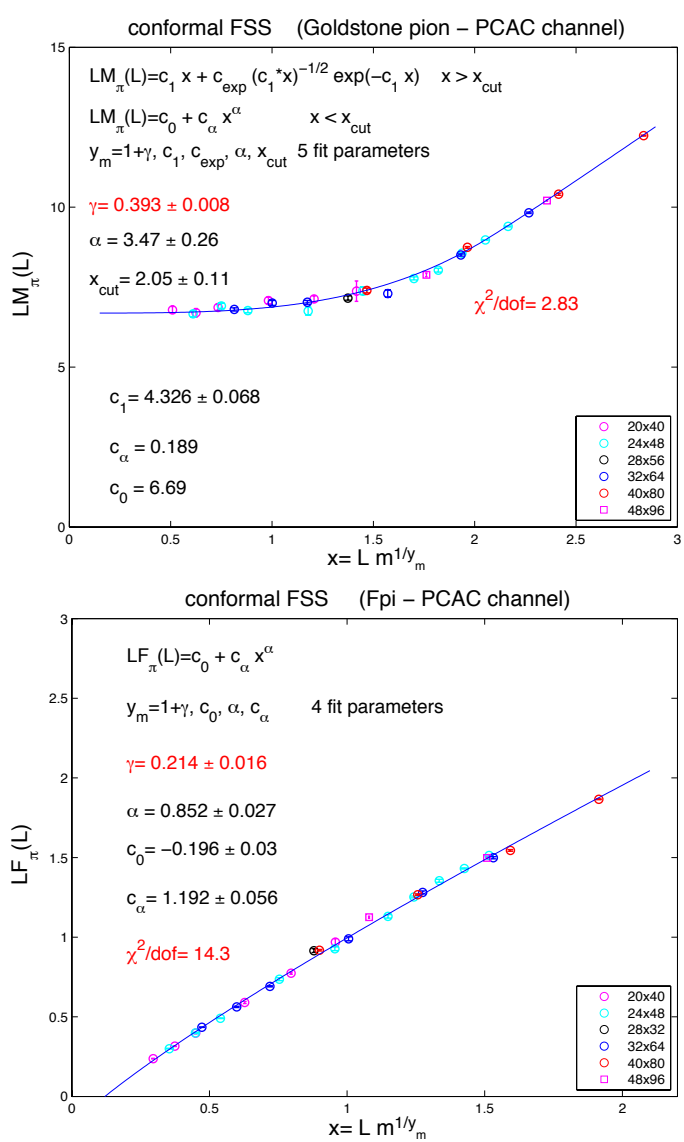

Fig. 1.5: Conformal FSS fits in four different quantum number channels. The fits are performed in each channel separately. Since the $\gamma$ values vary considerably from channel to channel, a simultaneous global fit to the combined channels with the same $\gamma$ exponent, as required by conformal FSS theory, is bound to fail.

color representation. In the fermion mass range $m=0.006-0.035$ with lattice sizes ranging in the fits from $20^{3} \times 40$ to $48^{3} \times 96$, two different FSS fitting procedures were applied. In the first procedure, a scaling function $f(x)$ for each mass $\mathrm{M}$ with five independent fitting parameters was defined. The fitting function $f(x)$ was divided into two regions separated at the joint $x=x_{\text {cut }}$. Different forms were chosen on the two sides of $x_{c u t}$ from the expected conformal behavior. For large $x>x_{c u t}$, the function $f(x)=c_{1} x+c_{\text {exp }}\left(c_{1} x\right)^{-1 / 2} \exp \left(-c_{1} x\right)$ 
with parameters $c_{1}$ and $c_{\text {exp }}$ describes the $L$-independent limit $M \sim c_{1} m^{1 / 1+\gamma}$ at fixed $m$ and $L \rightarrow \infty$. The $c_{\text {exp }}$ amplitude sets the size of the leading small exponential correction from the finite volume effect of the lightest pion state wrapping around the spatial volume. Since $f(0)=c_{0}$ is expected from conformal FSS with some power corrections at small $x$, we applied the simple ansatz $f(x)=c_{0}+c_{\alpha} x^{\alpha}$ for $x<x_{\text {cut }}$ (a more general polynomial function in the small $x$ region is not expected to change the conclusions from the fits). From the fit to the pion channel the parameter $c_{\pi}=c_{1}$ was determined and used as input in the exponential terms of the other channels with $\exp \left(-\mathrm{c}_{\pi} \mathrm{L}\right)$. The critical exponent $\gamma$ was included among the five fitting parameters, in addition to $c_{0}, c_{1}, c_{\text {exp }}$, and $x_{c u t}$. Individual quantum number channels can be fitted with conformal scaling functions $f(x)$ as shown in Figure 1.5 but the values of the critical exponent $\gamma$ are incompatible across different channels. The required global conformal FSS fit will fail with a single exponent $\gamma$ across all quantum numbers. The other fitting procedure, based on general spline functions, is different from [22, 45] but addresses related issues. With their extended data set Kuti and collaborators were unable to reproduce results in $[22,45]$ which used tables from their earlier limited subset of data [60]. It is important to emphasize that Kuti and collaborators have not reached definitive conclusions about the failure of conformal tests in the model. Much works remains to be done to use this method for definitive results in conformal and nearly conformal models.

\section{The scalar $\mathrm{f}_{0}$ (Higgs?) state in the $0^{++}$channel of BSM gauge theories}

It will take a large effort to understand, if four-dimensional strongly interacting gauge theories can replace the elementary scalar Higgs field. Common to all models is the determination of the scalar mass of the $f_{0}$ meson in the $0^{++}$channel. Kuti is proposing to investigate this problem in the sextet model first. This model will compete with alternatives from the theory space of Fig. 1.1, like models built on the $\mathrm{SU}(2)$ color gauge groups with fermions in the fundamental representation. All lessons learned will be applicable in a broad class of BSM gauge theories.

The properties of the $f_{0}$ meson are determined from staggered correlator I in Table 1 of [120]. The non-Goldstone scPion in staggered fermion terminology and the $f_{0}$ meson are parity partner states in this correlator. The quantum numbers of the $f_{0}$ meson match that of the $0^{++}$state in the staggered correlator. Staggered fermions with stout smearing are proposed to reduce the enormous computational resources required for this problem. Close to the conformal window the $f_{0}$ meson is not expected to be similar to the $\sigma$ particle of QCD.

To study flavor-singlet mesons, fermion loops are needed which are disconnected (often called hairpin diagrams). To evaluate disconnected fermion loops with zero momentum, sum over propagators is needed from sources at each spatial location for a given time slice. To reduce the enormously costly $\mathcal{O}(V)$ fermion matrix inversions to compute all-to-all propagators in lattice terminology, random sources have to be used with noise reduction. Staggered fermions with broken taste symmetry present an additional complication but it is well-known how to deal with the problem [29]. A very interesting further challenge and complication is the existence of two types of distinct $0^{++}$scalar mesons. One of them is the composite fermion state and the other is the scalar glueball with the same vacuum quantum numbers. In dynamical sextet simulations, these two types of state will mix which will require a well-chosen variational operator set to disentangle the scalar states. 


\section{Postdocs}

This section briefly describes work done by students and postdocs supported by this grant which resulted in publications in which none of the PIs is listed as a co-author.

\section{Pirtskhalava on General Relativity and Cosmology}

UCSD postdoc Dr. David Pirtskhalava explored aspects of gravity, cosmology, the cosmological constant problem, and modifications of gravity, including massive gravity. This resulted in the following publications over the last three years of the grant $[43,44,78,79$, $136]$

Unfortunately, the reduced funding level from the DOE, in our current cycle, has forced us to end Dr. Pirtskhalava's postdoctoral appointment at UCSD a year earlier than anticipated. He was able to find a postdoctoral appointment in Europe, which fortunately seems to be more steadily committed, at least at present, to the energy and cosmic frontiers.

The abstracts of Dr. Pirtskhalava's works on gravity and cosmology are as follows:

Ref. [78]: Solutions to scalar theories with derivative self-couplings often have regions where non-linearities are important. Given a classical source, there is usually a region, demarcated by the Vainshtein radius, inside of which the classical non-linearities are dominant, while quantum effects are still negligible. If perturbation theory is used to find such solutions, the expansion generally breaks down as the Vainshtein radius is approached from the outside. Here we show that it is possible, by integrating in certain auxiliary fields, to reformulate these theories in such a way that non-linearities become small inside the Vainshtein radius, and large outside it. This provides a complementary, or classically dual, description of the same theory - one in which non-perturbative regions become accessible perturbatively. We consider a few examples of classical solutions with various symmetries, and find that in all the cases the dual formulation makes it rather simple to study regimes in which the original perturbation theory fails to work. As an illustration, we reproduce by perturbative calculations some of the already known non-perturbative results, for a point-like source, cosmic string, and domain wall, and derive a new one. The dual formulation may be useful for developing the PPN formalism in the theories of modified gravity that give rise to such scalar theories.

Ref. [43]:General Relativity (GR), with or without matter fields, admits a natural extension to a scale invariant theory that requires a dilaton. Here we show that the recently formulated massive GR, minimally coupled to matter, possesses a new global symmetry related to scaling of the reference coordinates w.r.t. the physical ones. The field enforcing this symmetry, dubbed here quasi-dilaton, coincides with an ordinary dilaton if only pure gravity is considered, but differs from it when the matter Lagrangian is present. We study: (1) Theoretical consistency of massive GR with the quasi-dilaton; (2) Consistency with observations for spherically symmetric sources on (nearly) flat backgrounds; (3) Cosmological implications of this theory. We find that: (I) The theory with the quasi-dilaton is as consistent as massive GR is. (II) The Vainshtein mechanism is generically retained, owing to the fact that in the decoupling limit there is an enhanced symmetry, which turns the quasi-dilaton into a second galileon, consistently coupled to a tensor field. (III) Unlike in massive GR, there exist flat FRW solutions. In particular, we find self-accelerated solutions and discuss their quadratic perturbations. These solutions are testable by virtue of the different effective Newton's constants that govern the Hubble expansion and structure growth. 
Ref. [79]: Coupling the galileons to a curved background has been a tradeoff between maintaining second order equations of motion, maintaining the galilean shift symmetries, and allowing the background metric to be dynamical. We propose a construction which can achieve all three for a novel class of galilean invariant models, by coupling a scalar with the galilean symmetry to a massive graviton. This generalizes the brane construction for galileons, by adding to the brane a dynamical metric, (non-universally) interacting with the galileon field. Alternatively, it can be thought of as an extension of the ghost-free massive gravity, or as a massive graviton-galileon scalar-tensor theory. In the decoupling limit of these theories, new kinds of galileon invariant interactions arise between the scalar and the longitudinal mode of the graviton. These have higher order equations of motion and infinite powers of the field, yet are ghost-free.

Ref. [136]: We study the renormalization of some dimension-4, 7 and 10 operators in a class of nonlinear scalar-tensor theories. These theories are invariant under: (a) linear diffeomorphisms which represent an exact symmetry of the full non-linear action, and (b) global field-space Galilean transformations of the scalar field. The Lagrangian contains a set of non-topological interaction terms of the above-mentioned dimensionality, which we show are not renormalized at any order in perturbation theory. We also discuss the renormalization of other operators, that may be generated by loops and/or receive loop-corrections, and identify the regime in which they are sub-leading with respect to the operators that do not get renormalized. Interestingly, such scalar-tensor theories emerge in a certain high-energy limit of the ghost-free theory of massive gravity. One can use the non-renormalization properties of the high-energy limit to estimate the magnitude of quantum corrections in the full theory. We show that the quantum corrections to the three free parameters of the model, one of them being the graviton mass, are strongly suppressed. In particular, we show that having an arbitrarily small graviton mass is technically natural.

Ref. [44]: A theory of the quasidilaton is an extension of massive gravity by a scalar field, nonlinearly realizing a certain new global symmetry of the Lagrangian. It has been shown that unlike pure massive gravity, this theory does admit homogeneous and isotropic spatially flat solutions. Among the latter, self-accelerated solutions attract a special attention. Previous studies of perturbations, performed in the decoupling limit, revealed one healthy scalar mode, while the second relevant scalar was not captured in that limit. Here we study full cosmological perturbations above the simplest selfaccelerated background. We show that the fluctuations of a mixed state of the quasidilaton and the helicity- 0 graviton necessarily have a negative kinetic term at short distances, making this background unphysical. In addition, these cosmologies exhibit an order one sensitivity to higher dimensional terms suppressed by an energy scale that is parametrically higher than the strong coupling scale of the quasidilaton effective theory: such terms include Galileons, Goldstone-like selfinteractions and derivatives of the quasidilaton coupled to curvature, none of which introduce extra Ostrogradsky states. As one consequence, cosmology at the Hubble distances for this particular class of solutions depends on an unknown extension of the quasidilaton below its strong coupling distance scale. We note that non-FRW solutions that are similar to those of pure massive gravity should not necessarily suffer from these pathologies.

Ref. [80]: The unique ghost-free mass and nonlinear potential terms for general relativity are presented in a diffeomorphism and local Lorentz invariant vierbein formalism. This construction requires an additional two-index Stuckelberg field, beyond the four scalar fields 
used in the metric formulation, and unveils a new local SL(4) symmetry group of the mass and potential terms, not shared by the Einstein-Hilbert term. The new field is auxiliary but transforms as a vector under two different Lorentz groups, one of them the group of local Lorentz transformations, the other an additional global group. This formulation enables a geometric interpretation of the mass and potential terms for gravity in terms of certain volume forms. Furthermore, we find that the decoupling limit is much simpler to extract in this approach; in particular, we are able to derive expressions for the interactions of the vector modes. We also note that it is possible to extend the theory by promoting the twoindex auxiliary field into a Nambu-Goldstone boson nonlinearly realizing a certain space-time symmetry, and show how it is "eaten up" by the antisymmetric part of the vierbein.

In the Littlest Higgs model the Higgs is one of several pseudo-Goldstone bosons of an approximate global $S U(5)$ symmetry. The Higgs remains light compared to other pseudoGoldstone because it is protected by collective symmetry, where more than one operator is required to break the symmetry that protect the Higgs mass. However, in variations of the Littlest Higgs (e.g.. a model with only one $U(1)$ gauge group [130] or models with larger global symmetry) there are additional exact Goldstone bosons which cannot be lifted without violating collective symmetry. Hence there is a tension in lifting these light modes and preserving the lightness of the Higgs mass. Graduate student Patipan Uttayarat and postdoc Ze'ev Surujon showed[139] that once they incorporate explicit symmetry breaking, the new pseudo-Goldstone bosons of the LLH model can generally develop a vev with nontrivial phase. They then study the effect of this non-trivial phase in the low energy effective theory involving the Higgs and standard model quarks [117]. They find that for the model with one gauged $U(1)$ subgroup, even though there is a non-trivial phase, it doesn't produce a significant $\mathrm{CP}$ violating phase in the low energy effective theory. However, they point out, this is due to the minimal structure of the Littlest Higgs and then go on to display an extension of the model, based on the co-set $S U(6) / S O(6)$ model, where an $\mathcal{O}(1) \mathrm{CP}$ violating phase is generic. The presence of new scalar bosons in this model can lead to a novel decay channel for the Higgs. They demonstrated that in both the Littlest Higgs with one $U(1)$ and the $S U(6) / S O(6)$, the Higgs boson can have a large branching ratio into a pair of these new scalars for the low Higgs mass range.

\section{Flavor Physics}

Grinstein's students Stone and Uttayarat found an interesting connection between NP models that explain $A_{F B}^{t \bar{t}}$ and Grand Unified Theories (GUT) [138]. They consider a model that includes light colored scalars from the $\mathbf{4 5}$ and 50 representations of SU(5). These light scalars are, labeled by their charges under the Standard Model gauge groups, the $(6,1)_{4 / 3}$ and $(\overline{6}, 3)_{1 / 3}$ from the 50 and the $(8,2)_{1 / 2}$ from the $\mathbf{4 5}$. When the Yukawa coupling of the 50 is reasonably chosen and that of the $\mathbf{4 5}$ kept negligible at the scale of $M_{Z}$, the model yields phenomenologically viable results in agreement with the total $A_{F B}^{t \bar{t}}$ reported by CDF at the $0.7 \sigma$ level and with $A_{F B}^{t \bar{t}}\left(M_{t \bar{t}} \geq 450 \mathrm{GeV}\right)$ at the $2.2 \sigma$ level. Additionally, the Yukawa coupling of the $\mathbf{5 0}$ remains perturbative to the GUT scale (which defines a "reasonably chosen" value at $M_{Z}$ ), and the presence of the light scalar from the 45 allows for gauge coupling unification at a scale of $M_{G U T} \sim 10^{17} \mathrm{GeV}$.

Recently the LHCb collaboration published, and CDF confirmed, evidence for the difference between the time-integrated CP asymmetries in the two $D^{0}$ meson decay modes, $D^{0} \rightarrow$ $K^{+} K^{-}$and $D^{0} \rightarrow \pi^{+} \pi^{-}, A_{C P}\left(D^{0} \rightarrow K^{+} K^{-}\right)-A_{C P}\left(D^{0} \rightarrow \pi^{+} \pi^{-}\right)=-(0.82 \pm 0.21 \pm 0.11)$ 
$[1,4]$. It is commonly believed that the amount of CP violation (CPV) in $D$ decays is small within the Standard Model (SM) and any appreciable CPV effects would be an indication of new physics. Nevertheless, what is meant by 'small' is uncertain because we lack tools to reliably calculate matrix elements in QCD. Two decades ago Quigg carried out an $S U(3)$ analysis of $D$ decay amplitudes, neglecting CP violation [134], and Grinstein extended the analysis to incorporate CPV effects and short distance QCD corrections [82]. He found that in the $S U(3)$ limit the $D^{0}$ decay amplitudes into $K^{+} K^{-}$and $\pi^{+} \pi^{-}$could account for the the observed ratio of the partial rates $\Gamma\left(D^{0} \rightarrow K^{+} K^{-}\right) / \Gamma\left(D^{0} \rightarrow \pi^{+} \pi^{-}\right) \simeq 2.8$ only if the asymmetries $A_{C P}\left(D^{0} \rightarrow K^{+} K^{-}\right) \approx-A_{C P}\left(D^{0} \rightarrow \pi^{+} \pi^{-}\right)$are nearly of unit magnitude. This result requires an enhancement of about three orders of magnitude in one non-perturbative matrix element. Grinstein conjectured that accounting for $S U(3)$ breaking effects would reduce the required enhancement but predicted that a large CPV asymmetry would still occur. At Grinstein's suggestion, postdoc Pirtskhalava and student Uttayarat carried out a systematic flavor $S U(3)$ analysis of $D$-meson decays including the leading order symmetry breaking effects due to the nonzero strange quark mass $m_{s}$ [132]. They found that $S U(3)$ breaking can easily account for the recent $\mathrm{LHCb}$ measurements if the enhancement in the amplitudes is similar to that of the $\Delta=1 / 2$ rule in neutral kaon decays. As a byproduct of the analysis, they make certain predictions regarding the individual $K^{+} K^{-}$and $\pi^{+} \pi^{-}$ asymmetries as well as in the $D^{0} \rightarrow \pi^{0} \pi^{0}$ decay channel.

UCSD postdoc Ze'ev Surujon, under Grinstein's supervision, and collaborators[42] considered warped extra dimensional models with flavor alignment suppression of flavor violation. The alignment is achieved by partial gauging of the flavor symmetries in the bulk, and it is shown that this succeeds in reducing flavor violations to the point where the KK scale can be as low as order few $\mathrm{TeV}$. This works by initial alignment of bulk masses with down type Yukawa couplings, but then radiative corrections from the up-type Yukawas can induce problematic flavor misalignment for the down Yukawas. It is noted that this leads to flavor changing effects that are at around the current upper experimental bounds. General aspects of bounds on UV-brane induced flavor violating effects are presented. 


\section{References}

[1] R. Aaij et al. "Evidence for CP violation in time-integrated $D^{0} \rightarrow h^{-} h^{+}$decay rates". Phys.Rev.Lett. 108 (2012), p. 111602. DOI: 10.1103/PhysRevLett. 108.111602. arXiv: 1112.0938 [hep-ex].

[2] T. Aaltonen et al. "Search for Maximal Flavor Violating Scalars in Same-Charge Lepton Pairs in $p \bar{p}$ Collisions at $\sqrt{s}=1.96-T e V "$. Phys.Rev.Lett. 102 (2009), p. 041801. DOI: 10.1103/PhysRevLett.102.041801. arXiv: 0809.4903 [hep-ex].

[3] T. Aaltonen et al. "Evidence for a Mass Dependent Forward-Backward Asymmetry in Top Quark Pair Production". Phys.Rev. D83 (2011), p. 112003. DOI: 10.1103/ PhysRevD.83.112003. arXiv: 1101.0034 [hep-ex].

[4] T. Aaltonen et al. "Measurement of the difference of CP-violating asymmetries in $D^{0} \rightarrow K^{+} K^{-}$and $D^{0} \rightarrow \pi^{+} \pi^{-}$decays at CDF" (2012). arXiv: 1207.2158 [hep-ex].

[5] V. M. Abazov et al. "Forward-backward asymmetry in top quark-antiquark production". Phys.Rev. D84 (2011), p. 112005. DOI: 10.1103/PhysRevD.84.112005. arXiv: 1107.4995 [hep-ex].

[6] V. Abazov et al. "Observation and properties of the $X(3872)$ decaying to $J / \psi \pi^{+} \pi^{-}$ in $p \bar{p}$ collisions at $\sqrt{s}=1.96 \mathrm{TeV}$ ". Phys.Rev.Lett. 93 (2004), p. 162002. DOI: 10 . 1103/PhysRevLett.93.162002. arXiv: hep-ex/0405004 [hep-ex].

[7] D. E. Acosta et al. "Observation of the narrow state $X(3872) \rightarrow J / \psi \pi^{+} \pi^{-}$in $\bar{p} p$ collisions at $\sqrt{s}=1.96 \mathrm{TeV}$ ". Phys.Rev.Lett. 93 (2004), p. 072001. DOI: 10.1103 / PhysRevLett.93.072001. arXiv: hep-ex/0312021 [hep-ex].

[8] P. Agarwal, A. Amariti, and M. Siani. "Refined Checks and Exact Dualities in Three Dimensions" (2012). arXiv: 1205.6798 [hep-th].

[9] C. Alcock et al. "Possible gravitational microlensing of a star in the Large Magellanic Cloud". Nature 365 (1993), pp. 621-623. DOI: 10.1038/365621a0.

[10] C. Alcock et al. "EROS and MACHO combined limits on planetary mass dark matter in the galactic halo". Astrophys.J.Lett. (1998). arXiv: astro-ph/9803082 [astro-ph].

[11] C. Alcock et al. "The MACHO project: Microlensing results from 5.7 years of LMC observations". Astrophys.J. 542 (2000), pp. 281-307. DOI: 10.1086/309512. arXiv: astro-ph/0001272 [astro-ph].

[12] E. Alvarez et al. "Electroweak precision constraints on the Lee-Wick Standard Model". JHEP 04 (2008), p. 026. DOI: 10.1088/1126-6708/2008/04/026. arXiv: 0802.1061 [hep-ph].

[13] A. Amariti. "Dynamical SUSY Breaking and the Beta-Deformation". JHEP 1109 (2011), p. 111. DOI: 10.1007/JHEP09 (2011)111. arXiv: 1012.3154 [hep-th]. 
[14] A. Amariti, D. Forcella, and A. Mariotti. "Additional Light Waves in Hydrodynamics and Holography" (2010). arXiv: 1010.1297 [hep-th].

[15] A. Amariti and S. Franco. "Free Energy vs Sasaki-Einstein Volume for Infinite Families of M2-Brane Theories" (2012). arXiv: 1204.6040 [hep-th].

[16] A. Amariti and K. Intriligator. "(Delta a) curiosities in some 4d susy RG flows". JHEP 1211 (2012), p. 108. DOI: 10.1007 / JHEP11(2012) 108. arXiv: 1209.4311 [hep-th].

[17] A. Amariti, C. Klare, and M. Siani. "The Large N Limit of Toric Chern-Simons Matter Theories and Their Duals" (2011). arXiv: 1111.1723 [hep-th].

[18] A. Amariti and M. Siani. "Z-extremization and F-theorem in Chern-Simons matter theories". JHEP 10 (2011), p. 016. DOI: 10.1007/JHEP10(2011)016. arXiv: 1105 . 0933 [hep-th].

[19] A. Amariti and D. Stone. "Spontaneous R-symmetry breaking from the renormalization group flow". JHEP 1301 (2013), p. 092. DOI: 10.1007/JHEP01(2013)092. arXiv: 1210.3028 [hep-th].

[20] P. del Amo Sanchez et al. "Evidence for the decay $X(3872) \rightarrow J / \psi \omega "$ ". Phys.Rev. D82 (2010), p. 011101. DOI: 10.1103/PhysRevD.82.011101. arXiv: 1005.5190 [hep-ex].

[21] T. Appelquist and Y. Bai. "A Light Dilaton in Walking Gauge Theories". Phys.Rev. D82 (2010), p. 071701. DOI: 10.1103/PhysRevD . 82 .071701. arXiv: 1006. 4375 [hep-ph].

[22] T. Appelquist et al. "Lattice Simulations and Infrared Conformality". Phys.Rev. D84 (2011), p. 054501. DOI: 10 . 1103 / PhysRevD . 84 .054501. arXiv: 1106 . 2148 [hep-lat].

[23] N. Arkani-Hamed et al. "The Littlest Higgs". JHEP 0207 (2002), p. 034. arXiv: hep-ph/0206021 [hep-ph].

[24] P. Artoisenet and E. Braaten. "Production of the $X(3872)$ at the Tevatron and the LHC". Phys.Rev. D81 (2010), p. 114018. DOI: 10.1103/PhysRevD.81.114018. arXiv: 0911.2016 [hep-ph].

[25] B. Aubert et al. "Study of the $B \rightarrow J / \psi K^{-} \pi^{+} \pi^{-}$decay and measurement of the $B \rightarrow X(3872) K^{-}$branching fraction". Phys.Rev. D71 (2005), p. 071103. DoI: 10 . 1103/PhysRevD.71.071103. arXiv: hep-ex/0406022 [hep-ex].

[26] S. Bailey, K. Griest, et al. "Addressing Decadal Survey Science through Community Access to Highly Multiplexed Spectroscopy with BigBOSS on the Mayall Telescope". White Paper for 2012 NSF Portfolio Review 000 (2012), p. 000. DOI: 000.

[27] T. Banks and A. Zaks. "On the Phase Structure of Vector-Like Gauge Theories with Massless Fermions". Nucl.Phys. B196 (1982), p. 189. DOI: 10.1016/0550-3213(82) 90035-9.

[28] W. A. Bardeen, C. N. Leung, and S. Love. "The Dilaton and Chiral Symmetry Breaking". Phys.Rev.Lett. 56 (1986), p. 1230. DOI: 10.1103/PhysRevLett.56.1230. 
[29] C. Bernard et al. "Scalar meson spectroscopy with lattice staggered fermions". Phys.Rev. D76 (2007), p. 094504. DOI: 10 . 1103/PhysRevD . 76 .094504. arXiv: 0707.2402 [hep-lat].

[30] C. Bignamini et al. "Prompt production of loosely bound mesonic molecules at hadron colliders". PoS DIS2010 (2010), p. 176.

[31] C. Bignamini et al. "Is the X(3872) molecular hypothesis compatible with CDF data?" PoS EPS-HEP2009 (2009), p. 074.

[32] C. Bignamini et al. "Is the X(3872) Production Cross Section at Tevatron Compatible with a Hadron Molecule Interpretation?" Phys.Rev.Lett. 103 (2009), p. 162001. DOI: 10.1103/PhysRevLett.103.162001. arXiv: 0906.0882 [hep-ph].

[33] C. Bignamini et al. "More loosely bound hadron molecules at CDF?" Phys.Lett. B684 (2010), pp. 228-230. DOI: 10.1016/j ·physletb.2010.01.037. arXiv: 0912.5064 [hep-ph].

[34] F. Brazzi et al. "Strong Couplings of $X(3872)_{J=1,2}$ and a New Look at $J / \psi$ Suppression in Heavy Ion Collisions". Phys.Rev. D84 (2011), p. 014003. DOI: 10.1103/ PhysRevD.84.014003. arXiv: 1103.3155 [hep-ph].

[35] C. D. Carone and R. F. Lebed. "Minimal Lee-Wick Extension of the Standard Model". Phys. Lett. B668 (2008), pp. 221-225. DOI: $10.1016 /$ j . physletb. 2008 . 08 . 050. arXiv: 0806.4555 [hep-ph].

[36] W. E. Caswell. "Asymptotic Behavior of Nonabelian Gauge Theories to Two Loop Order". Phys.Rev.Lett. 33 (1974), p. 244. DOI: 10.1103/PhysRevLett.33.244.

[37] R. S. Chivukula et al. "Custodial Isospin Violation in the Lee-Wick Standard Model". Phys. Rev. D81 (2010), p. 095015. DOI: 10 . 1103 / PhysRevD . 81 . 095015. arXiv: 1002.0343 [hep-ph].

[38] R. Chivukula et al. "Global Symmetries and Renormalizability of Lee-Wick Theories". Phys.Rev. D82 (2010), p. 035015. DOI: 10.1103/PhysRevD.82.035015. arXiv: 1006. 2800 [hep-ph].

[39] R. Chivukula et al. "HIGGS DECAY INTO GOLDSTONE BOSONS". Annals Phys. 192 (1989), pp. 93-103. DOI: $10.1016 / 0003-4916$ (89)90119-X.

[40] S. Choi et al. "Observation of a narrow charmonium - like state in exclusive $B^{ \pm} \rightarrow$ $K^{ \pm} p i+p i-J / \psi$ decays". Phys.Rev.Lett. 91 (2003), p. 262001. DOI: $10.1103 /$ PhysRevLett.91.262001. arXiv: hep-ex/0309032 [hep-ex].

[41] A. M. Cieplak and K. Griest. "Improved Theoretical Predictions of Microlensing Rates for the Detection of Primordial Black Hole Dark Matter". Astrophys.J. 767 (2013), p. 145. DOI: 10.1088/0004-637X/767/2/145. arXiv: 1210.7729 [astro-ph.CO] .

[42] C. Csaki et al. "Flavor Alignment via Shining in RS". Phys.Rev. D81 (2010), p. 075025. DOI: 10.1103/PhysRevD.81.075025. arXiv: 0907.0474 [hep-ph].

[43] G. D'Amico et al. "Quasi-Dilaton: Theory and Cosmology" (2012). arXiv: 1206.4253 [hep-th]. 
[44] G. D'Amico et al. "On Cosmological Perturbations of Quasidilaton" (2013). arXiv: 1304.0723 [hep-th].

[45] T. DeGrand. "Finite-size scaling tests for spectra in SU(3) lattice gauge theory coupled to 12 fundamental flavor fermions". Phys.Rev. D84 (2011), p. 116901. Dor: 10. 1103/PhysRevD.84.116901. arXiv: 1109.1237 [hep-lat].

[46] T. DeGrand, Y. Shamir, and B. Svetitsky. "Mass anomalous dimension in sextet QCD” (2012). arXiv: 1201.0935 [hep-lat].

[47] L. Del Debbio et al. "The infrared dynamics of Minimal Walking Technicolor". Phys.Rev. D82 (2010), p. 014510. DOI: 10.1103 / PhysRevD . 82 .014510. arXiv: 1004.3206 [hep-lat].

[48] D. D. Dietrich and F. Sannino. "Conformal window of SU(N) gauge theories with fermions in higher dimensional representations". Phys.Rev. D75 (2007), p. 085018. DOI: 10.1103/PhysRevD.75.085018. arXiv: hep-ph/0611341 [hep-ph].

[49] T. R. Dulaney and M. B. Wise. "Flavor Changing Neutral Currents in the Lee-Wick Standard Model". Phys. Lett. B658 (2008), pp. 230-235. DOI: 10.1016/j . physletb. 2007.08.049. arXiv: 0708.0567 [hep-ph].

[50] J. Ellis and T. You. "Global Analysis of the Higgs Candidate with Mass 125 GeV" (2012). arXiv: 1207.1693 [hep-ph].

[51] J. R. Espinosa et al. "Neutrino masses in the Lee-Wick standard model". Phys. Rev. D77 (2008), p. 085002. DOI: 10.1103/PhysRevD . 77 .085002. arXiv: 0705 . 1188 [hep-ph].

[52] J. Espinosa and B. Grinstein. "Ultraviolet Properties of the Higgs Sector in the LeeWick Standard Model". To appear in Physical Review D (2011). arXiv: 1101.5538 [hep-ph].

[53] Z. Fodor et al. "Calculating the running coupling in strong electroweak models". PoS LAT2009 (2009), p. 058. arXiv: 0911.2934 [hep-lat].

[54] Z. Fodor et al. "Chiral properties of SU(3) sextet fermions". JHEP 0911 (2009), p. 103. DOI: 10.1088/1126-6708/2009/11/103. arXiv: 0908.2466 [hep-lat].

[55] Z. Fodor et al. "Chiral symmetry breaking in nearly conformal gauge theories". PoS LAT2009 (2009), p. 055. arXiv: 0911.2463 [hep-lat].

[56] Z. Fodor et al. "Nearly conformal gauge theories in finite volume". Phys.Lett. B681 (2009), pp. 353-361. DOI: 10.1016/j.physletb.2009.10.040. arXiv: 0907.4562 [hep-lat].

[57] Z. Fodor et al. "Topology and higher dimensional representations". JHEP 0908 (2009), p. 084. DOI: 10.1088/1126-6708/2009/08/084. arXiv: 0905.3586 [hep-lat].

[58] Z. Fodor et al. "Nearly conformal gauge theories on the lattice". Int.J.Mod.Phys. A25 (2010), pp. 5162-5174. DOI: 10.1142/S0217751X10050937.

[59] Z. Fodor et al. "Chiral symmetry breaking in fundamental and sextet fermion representations of SU(3) color" (2011). arXiv: 1103.5998 [hep-lat]. 
[60] Z. Fodor et al. "Twelve massless flavors and three colors below the conformal window". Phys.Lett. B703 (2011), pp. 348-358. DOI: 10.1016 / j . physletb . 2011 . 07 . 037. arXiv: 1104.3124 [hep-lat].

[61] Z. Fodor et al. "Can the nearly conformal sextet gauge model hide the Higgs impostor?" Phys.Lett. B718 (2012), pp. 657-666. DOI: 10.1016/j .physletb.2012 .10.079. arXiv: 1209.0391 [hep-lat].

[62] Z. Fodor et al. "Confining force and running coupling with twelve fundamental and two sextet fermions". PoS LATTICE2012 (2012), p. 025. arXiv: 1211.3548 [hep-lat].

[63] Z. Fodor et al. "Lattice methods in gauge theories beyond the standard model". Prog.Theor.Phys.Suppl. 197 (2012), pp. 31-45. DOI: 10.1143/PTPS.197.31.

[64] Z. Fodor et al. "The gradient flow running coupling scheme". PoS LATTICE2012 (2012), p. 050. arXiv: 1211.3247 [hep-lat].

[65] Z. Fodor et al. "The sextet gauge model, light Higgs, and the dilaton". PoS LATTICE2012 (2012), p. 024. arXiv: 1211.6164 [hep-lat].

[66] Z. Fodor et al. "The Yang-Mills gradient flow in finite volume". JHEP 1211 (2012), p. 007. DOI: 10.1007/JHEP11 (2012)007. arXiv: 1208.1051 [hep-lat].

[67] Z. Fodor et al. "Twelve fundamental and two sextet fermion flavors" (2012). arXiv: 1205.1878 [hep-lat].

[68] B. Fornal, B. Grinstein, and M. B. Wise. "Lee-Wick Theories at High Temperature". Phys. Lett. B674 (2009), pp. 330-335. DOI: 10.1016/j ·physletb. 2009 . 03 . 036. arXiv: 0902.1585 [hep-th].

[69] J.-F. Fortin, B. Grinstein, and A. Stergiou. "Scale without Conformal Invariance: An Example". Phys. Lett. B704 (2011), pp. 74-80. DOI: 10.1016/j .physletb.2011.08. 060. arXiv: 1106.2540 [hep-th].

[70] J.-F. Fortin, B. Grinstein, and A. Stergiou. "Scale without Conformal Invariance at Three Loops" (2012). arXiv: 1202.4757 [hep-th].

[71] J.-F. Fortin, B. Grinstein, and A. Stergiou. "Scale without Conformal Invariance in Four Dimensions" (2012). arXiv: 1206.2921 [hep-th].

[72] J.-F. Fortin, B. Grinstein, and A. Stergiou. "Scale without Conformal Invariance: Theoretical Foundations". JHEP 1207 (2012), p. 025. DOI: 10.1007/ JHEP07 (2012) 025. arXiv: 1107.3840 [hep-th].

[73] J.-F. Fortin, B. Grinstein, and A. Stergiou. "Limit Cycles and Conformal Invariance". JHEP 1301 (2013), p. 184. DOI: 10.1007 / JHEP01(2013) 184. arXiv: 1208 . 3674 [hep-th].

[74] J.-F. Fortin, K. Intriligator, and A. Stergiou. "Current OPEs in Superconformal Theories". JHEP 09 (2011), p. 071. DOI: 10.1007/JHEP09(2011)071. arXiv: 1107.1721 [hep-th].

[75] J.-F. Fortin, K. Intriligator, and A. Stergiou. "Superconformally Covariant OPE and General Gauge Mediation". JHEP 12 (2011), p. 064. DOI: 10.1007 / JHEP12(2011) 064. arXiv: 1109.4940 [hep-th]. 
[76] J.-F. Fortin and A. Stergiou. "Field-theoretic Methods in Strongly-Coupled Models of General Gauge Mediation". Nucl.Phys. B873 (2013), pp. 92-115. DOI: 10.1016/j . nuclphysb.2013.04.009. arXiv: 1212.2202 [hep-th].

[77] J.-F. Fortin et al. "On Limit Cycles in Supersymmetric Theories". Phys.Lett. B719 (2013), pp. 170-173. DOI: 10.1016/j . physletb.2012.12.059. arXiv: 1210.2718 [hep-th].

[78] G. Gabadadze, K. Hinterbichler, and D. Pirtskhalava. "Classical Duals of Derivatively Self-Coupled Theories". Phys.Rev. D85 (2012), p. 125007. DOI: 10.1103/PhysRevD. 85.125007. arXiv: 1202.6364 [hep-th].

[79] G. Gabadadze et al. "A Covariant Master Theory for Novel Galilean Invariant Models and Massive Gravity". Phys.Rev. D86 (2012), p. 124004. DOI: 10.1103 /PhysRevD . 86.124004. arXiv: 1208.5773 [hep-th].

[80] G. Gabadadze et al. "On the Potential for General Relativity and its Geometry" (2013). arXiv: 1307.2245 [hep-th].

[81] W. D. Goldberger, B. Grinstein, and W. Skiba. "Distinguishing the Higgs boson from the dilaton at the Large Hadron Collider". Phys.Rev.Lett. 100 (2008), p. 111802. DOI: 10.1103/PhysRevLett.100.111802. arXiv: 0708.1463 [hep-ph].

[82] M. Golden and B. Grinstein. "Enhanced CP Violations in Hadronic Charm Decays". Phys.Lett. B222 (1989), p. 501. DOI: 10.1016/0370-2693(89)90353-5.

[83] K. Griest. "Report on JDEM to the National Research Council" (). Report on JDEM to the National Research Council (July 2006).

[84] K. Griest and D. Seckel. "Cosmic Asymmetry, Neutrinos and the Sun". Nucl.Phys. B283 (1987), p. 681. DOI: 10.1016/0550-3213(87)90293-8.

[85] K. Griest. "Cross-Sections, Relic Abundance and Detection Rates for Neutralino Dark Matter". Phys.Rev. D38 (1988), p. 2357. DOI: 10.1103/PhysRevD . 38. 2357, 10.1103/ PhysRevD.39.3802.

[86] K. Griest et al. "Gravitational microlensing as a method of detecting disk dark matter and faint disk stars" (1990).

[87] K. Griest. "GALACTIC MICROLENSING AS A METHOD OF DETECTING MASSIVE COMPACT HALO OBJECTS". Astrophys.J. 366 (1991), pp. 412-421. DOI: $10.1086 / 169575$.

[88] K. Griest. "Toward a possible solution to the cosmic coincidence problem". Phys.Rev. D66 (2002), p. 123501. DOI: 10.1103/PhysRevD . 66 . 123501. arXiv: astro-ph / 0202052 [astro-ph].

[89] K. Griest et al. "Report of the Astronomy and Astrophysics Advisory Committee" (2011). Yearly Report to DOE, NASA, NSF, and Congress, March 15, 2011, pp. 1-32.

[90] K. Griest. "Dark Matter and Dark Energy: Working with the Dark Side to Discover the Ultimate Fate of the Universe" (2012). HiPACC Conference, Computational Astronomy: From Planets to Cosmos, UC Santa Cruz, June, 2012. 
[91] K. Griest. "Dark Matter, Dark Energy, and the Ultimate Fate of the Universe" (2013). Be Inspired: Engineering talk series, UCSD, Jan 2013.

[92] K. Griest. "Primordial Black Hole Dark Matter from Kepler Source Microlensing" (2013). SNOWDARK: non-SUSY Dark Matter, Snowbird, UT, March 2013.

[93] K. Griest and W. Hu. "Effect of binary sources on the search for MACHOs via microlensing". Astrophys.J. 397 (1992), p. 362. DOI: 10.1086/171793.

[94] K. Griest and M. Kamionkowski. "Unitarity Limits on the Mass and Radius of Dark Matter Particles". Phys.Rev.Lett. 64 (1990), p. 615. DOI: 10.1103/PhysRevLett. 64. 615.

[95] K. Griest, M. Kamionkowski, and M. S. Turner. "Supersymmetric Dark Matter Above the W Mass". Phys.Rev. D41 (1990), pp. 3565-3582. DOI: 10.1103/PhysRevD . 41. 3565 .

[96] K. Griest and E. W. Kolb. "Solitosynthesis: Cosmological Evolution of Nontopological Solitons". Phys.Rev. D40 (1989), p. 3231. DOI: 10.1103/PhysRevD .40.3231.

[97] K. Griest and N. Safizadeh. "The use of high magnification microlensing events in discovering extra-solar planets". Astrophys.J. 500 (1998), pp. 37-50. DOI: 10.1086/ 305729. arXiv: astro-ph/9710342 [astro-ph].

[98] K. Griest and D. Seckel. "Three exceptions in the calculation of relic abundances". Phys.Rev. D43 (1991), pp. 3191-3203. DOI: 10.1103/PhysRevD.43.3191.

[99] K. Griest et al. "Wavelength Accuracy of the Keck HIRES Spectrograph and Measuring Changes in the Fine Structure Constant". Astrophys.J. 708 (2010), pp. 158-170. DOI: $10.1088 / 0004-637 X / 708 / 1 / 158$. arXiv: 0904.4725 [astro-ph.CO].

[100] K. Griest et al. "Microlensing of Kepler Stars as a Method of Detecting Primordial Black Hole Dark Matter". Phys.Rev.Lett. 107 (2011). 5 pages, 1 figure, p. 231101. DOI: 10.1103/PhysRevLett.107.231101. arXiv: 1109.4975 [astro-ph.CO].

[101] B. Grinstein, L. J. Hall, and L. Randall. "Do B meson decays exclude a light Higgs?" Phys.Lett. B211 (1988), pp. 363-369. DOI: 10.1016/0370-2693(88)90916-1.

[102] B. Grinstein, R. Kelley, and P. Uttayarat. "Hidden fine tuning in the quark sector of little higgs models". JHEP 09 (2009), p. 040. DOI: 10.1088/1126-6708/2009/09/ 040. arXiv: 0904.1622 [hep-ph].

[103] B. Grinstein, R. Kelley, and P. Uttayarat. "One Loop Renormalization of the Littlest Higgs Model". JHEP 1102 (2011), p. 089. DOI: 10.1007/JHEP02(2011) 089. arXiv: 1011.0682 [hep-ph].

[104] B. Grinstein and C. W. Murphy. "Bottom Quark Forward-Backward Asymmetry in the Standard Model and Beyond" (2013). arXiv: 1302.6995 [hep-ph].

[105] B. Grinstein, C. W. Murphy, and M. Trott. "EWPD Constraints on Flavor Symmetric Vector Fields". JHEP 11 (2011), p. 139. DOI: 10.1007/ JHEP11(2011) 139. arXiv: 1110.5361 [hep-ph]. 
[106] B. Grinstein and D. O'Connell. "One-Loop Renormalization of Lee-Wick Gauge Theory". Phys. Rev. D78 (2008), p. 105005. DOI: 10.1103/PhysRevD.78.105005. arXiv: 0801.4034 [hep-ph].

[107] B. Grinstein, D. O'Connell, and M. B. Wise. "The Lee-Wick standard model". Phys. Rev. D77 (2008), p. 025012. DOI: 10.1103/PhysRevD.77.025012. arXiv: 0704.1845 [hep-ph].

[108] B. Grinstein, M. Redi, and G. Villadoro. "Low Scale Flavor Gauge Symmetries". JHEP 1011 (2010), p. 067. DOI: 10.1007 / JHEP11(2010) 067. arXiv: 1009 . 2049 [hep-ph].

[109] B. Grinstein and M. Trott. "A Higgs-Higgs bound state due to New Physics at a TeV". Phys. Rev. D76 (2007), p. 073002. DOI: 10.1103/PhysRevD. 76.073002. arXiv: 0704.1505 [hep-ph].

[110] B. Grinstein and M. Trott. "Electroweak Baryogenesis with a Pseudo-Goldstone Higgs". Phys. Rev. D78 (2008), p. 075022. DOI: 10 . 1103 / PhysRevD . 78 . 075022. arXiv: 0806.1971 [hep-ph].

[111] B. Grinstein and P. Uttayarat. "A Very Light Dilaton". JHEP 1107 (2011), p. 038. DOI: 10.1007/JHEP07 (2011)038. arXiv: 1105.2370 [hep-ph] .

[112] B. Grinstein et al. "Flavor Symmetric Sectors and Collider Physics". JHEP 1110 (2011), p. 072. DOI: 10.1007/JHEP10(2011)072. arXiv: 1108.4027 [hep-ph].

[113] B. Grinstein et al. "Forward-backward asymmetry in $t \bar{t}$ production from flavour symmetries". Phys.Rev.Lett. 107 (2011), p. 012002. DOI: 10.1103/PhysRevLett . 107. 012002. arXiv: 1102.3374 [hep-ph].

[114] B. Grinstein et al. "Massive Spin-2 States as the Origin of the Top Quark ForwardBackward Asymmetry". JHEP 1208 (2012), p. 073. DOI: 10.1007/JHEP08(2012) 073. arXiv: 1203.2183 [hep-ph].

[115] M. Hashimoto and K. Yamawaki. "Techni-dilaton at Conformal Edge". Phys.Rev. D83 (2011), p. 015008. DOI: 10.1103/PhysRevD.83.015008. arXiv: 1009.5482 [hep-ph].

[116] B. Holdom and J. Terning. "A Light Dilaton in Gauge Theories?" Phys.Lett. B187 (1987), p. 357. DOI: 10.1016/0370-2693(87)91109-9.

[117] S. J. Huber, M. Pospelov, and A. Ritz. "Electric dipole moment constraints on minimal electroweak baryogenesis". Phys.Rev. D75 (2007), p. 036006. DOI: 10 . 1103 / PhysRevD .75.036006. arXiv: hep-ph/0610003 [hep-ph].

[118] K. Intriligator and N. Seiberg. "Aspects of 3d N=2 Chern-Simons-Matter Theories" (2013). arXiv: 1305.1633 [hep-th].

[119] K. Intriligator et al. "Conifold Transitions in M-theory on Calabi-Yau Fourfolds with Background Fluxes" (2012). arXiv: 1203.6662 [hep-th].

[120] N. Ishizuka et al. "Operator dependence of hadron masses for Kogut-Susskind quarks on the lattice". Nucl.Phys. B411 (1994), pp. 875-902. DOI: 10.1016/0550-3213(94) 90475-8. 
[121] I. Jack and H. Osborn. "Analogs For The $c$ Theorem For Four-Dimensional Renormalizable Field Theories". Nucl.Phys. B343 (1990), pp. 647-688. DOI: 10.1016/05503213 (90) 90584-Z.

[122] G. Jungman, M. Kamionkowski, and K. Griest. "Supersymmetric dark matter". Phys.Rept. 267 (1996), pp. 195-373. DOI: 10.1016/0370-1573(95)00058-5. arXiv: hep-ph/9506380 [hep-ph].

[123] M. Kamionkowski et al. "Model independent comparison of direct versus indirect detection of supersymmetric dark matter". Phys.Rev.Lett. 74 (1995), pp. 5174-5177. DOI: 10.1103/PhysRevLett.74.5174. arXiv: hep-ph/9412213 [hep-ph].

[124] I. Low, J. Lykken, and G. Shaughnessy. "Have We Observed the Higgs (Imposter)?" (2012). arXiv: 1207.1093 [hep-ph].

[125] M. Luscher et al. "The Schrodinger functional: A Renormalizable probe for nonAbelian gauge theories". Nucl.Phys. B384 (1992), pp. 168-228. DOI: 10.1016/05503213(92) 90466-0. arXiv: hep-lat/9207009 [hep-lat].

[126] N. Mekareeya, J. Song, and Y. Tachikawa. "2d TQFT structure of the superconformal indices with outer-automorphism twists". JHEP 1303 (2013), p. 171. DOI: 10.1007/ JHEP03 (2013) 171. arXiv: 1212.0545 [hep-th].

[127] M. T. Murphy, J. Webb, and V. Flambaum. "Further evidence for a variable finestructure constant from Keck/HIRES QSO absorption spectra". Mon.Not.Roy.Astron.Soc. 345 (2003), p. 609. DOI: $10.1046 / \mathrm{j} .1365-8711.2003 .06970$. x. arXiv: astro$\mathrm{ph} / 0306483$ [astro-ph].

[128] B. Paczynski. "Gravitational microlensing by the galactic halo". Astrophys.J. 304 (1986), pp. 1-5. DOI: 10.1086/164140.

[129] D. S. Park and J. Song. "The Seiberg-Witten Kahler Potential as a Two-Sphere Partition Function". JHEP 1301 (2013), p. 142. DOI: 10.1007 / JHEP01(2013) 142. arXiv: 1211.0019 [hep-th].

[130] M. Perelstein, M. E. Peskin, and A. Pierce. "Top quarks and electroweak symmetry breaking in little Higgs models". Phys.Rev. D69 (2004), p. 075002. DOI: 10.1103 / PhysRevD .69.075002. arXiv: hep-ph/0310039 [hep-ph].

[131] C. Pilachowski et al. "Addressing Decadal Survey Science through Community Access to Highly Multiplexed Spectroscopy with BigBOSS on the KPNO Mayall Telescope" (2012). arXiv: 1211.0285 [astro-ph.CO].

[132] D. Pirtskhalava and P. Uttayarat. "CP Violation and Flavor SU(3) Breaking in Dmeson Decays" (2011). arXiv: 1112.5451 [hep-ph].

[133] J. Polchinski. "Scale And Conformal Invariance In Quantum Field Theory". Nucl.Phys. B303 (1988), p. 226. DOI: 10.1016/0550-3213(88)90179-4.

[134] C. Quigg. "Charmed Meson Decays and the Structure of the Charged Weak Current". Z.Phys. C4 (1980), p. 55. DOI: 10.1007/BF01477308.

[135] C. Quigg. "The Lost tribes of charmonium". Nucl.Phys.Proc.Suppl. 142 (2005), pp. 8797. DOI: 10.1016/j.nuclphysbps.2005.01.016. arXiv: hep-ph/0407124 [hep-ph]. 
[136] C. de Rham et al. "Non-Renormalization and Naturalness in a Class of Scalar-Tensor Theories" (2012). DOI: 10.1103/PhysRevD.87.085017. arXiv: 1212.4128 [hep-th].

[137] F. Sannino. "Conformal Dynamics for TeV Physics and Cosmology". Acta Phys.Polon. B40 (2009), pp. 3533-3743. arXiv: 0911.0931 [hep-ph].

[138] D. C. Stone and P. Uttayarat. "Explaining the ttbar Forward-Backward Asymmetry from a GUT-Inspired Model". JHEP 1201 (2012), p. 096. DOI: 10.1007/ JHEP01 (2012) 096. arXiv: 1111.2050 [hep-ph].

[139] Z. Surujon and P. Uttayarat. "Spontaneous CP Violation and Light Particles in The Littlest Higgs". Phys.Rev. D83 (2011), p. 076010. DOI: 10 . 1103 / PhysRevD . 83 . 076010. arXiv: 1003.4779 [hep-ph].

[140] T. E. J. Underwood and R. Zwicky. "Electroweak Precision Data and the Lee-Wick Standard Model". Phys. Rev. D79 (2009), p. 035016. DOI: 10.1103/PhysRevD. 79. 035016. arXiv: 0805.3296 [hep-ph].

[141] D. Wallace and R. Zia. "Gradient Properties of the Renormalization Group Equations in Multicomponent Systems". Annals Phys. 92 (1975), p. 142. DOI: 10.1016/00034916 (75) $90267-5$.

[142] J. B. Whitmore, M. T. Murphy, and K. Griest. "Wavelength Calibration of the VLTUVES Spectrograph". Astrophys.J. 723 (2010), pp. 89-99. DOI: 10 . 1088 /0004637X/723/1/89. arXiv: 1004.3325 [astro-ph. IM].

[143] K. G. Wilson. "The Renormalization Group and Strong Interactions". Phys.Rev. D3 (1971), p. 1818. DOI: 10.1103/PhysRevD.3.1818.

[144] K. Yamawaki, M. Bando, and K.-i. Matumoto. "Scale Invariant Technicolor Model and a Technidilaton". Phys.Rev.Lett. 56 (1986), p. 1335. DOI: 10.1103/PhysRevLett. 56.1335.

[145] A. Zamolodchikov. "Irreversibility of the Flux of the Renormalization Group in a 2D Field Theory". JETP Lett. 43 (1986), pp. 730-732. 


\section{Contract Publications}

Contract publications listed alphabetically and enumerated. The numbers in the list do not correspond to citations in the narrative.

[1] P. Agarwal, A. Amariti, and M. Siani. "Refined Checks and Exact Dualities in Three Dimensions" (2012). arXiv: 1205.6798 [hep-th].

[2] A. Amariti. "Dynamical SUSY Breaking and the Beta-Deformation". JHEP 1109 (2011), p. 111. DOI: 10.1007/JHEP09(2011)111. arXiv: 1012.3154 [hep-th].

[3] A. Amariti, D. Forcella, and A. Mariotti. "Additional Light Waves in Hydrodynamics and Holography" (2010). arXiv: 1010.1297 [hep-th].

[4] A. Amariti, D. Forcella, and A. Mariotti. "Negative Refractive Index in Hydrodynamical Systems" (2011). arXiv: 1107.1240 [hep-th].

[5] A. Amariti, D. Forcella, and A. Mariotti. "Integrability on the Master Space". JHEP 1206 (2012), p. 053. DOI: 10.1007/JHEP06(2012)053. arXiv: 1203.1616 [hep-th].

[6] A. Amariti and S. Franco. "Free Energy vs Sasaki-Einstein Volume for Infinite Families of M2-Brane Theories" (2012). arXiv: 1204.6040 [hep-th].

[7] A. Amariti and K. Intriligator. "(Delta a) curiosities in some 4d susy RG flows". JHEP 1211 (2012), p. 108. DOI: 10.1007 / JHEP11(2012) 108. arXiv: 1209.4311 [hep-th].

[8] A. Amariti, C. Klare, and M. Siani. "The Large N Limit of Toric Chern-Simons Matter Theories and Their Duals" (2011). arXiv: 1111.1723 [hep-th].

[9] A. Amariti and M. Siani. "Z-extremization and F-theorem in Chern-Simons matter theories". JHEP 10 (2011), p. 016. DOI: 10.1007/JHEP10(2011)016. arXiv: 1105. 0933 [hep-th].

[10] A. Amariti and D. Stone. "Spontaneous R-symmetry breaking from the renormalization group flow". JHEP 1301 (2013), p. 092. DOI: 10.1007/JHEP01(2013) 092. arXiv: 1210.3028 [hep-th].

[11] A. Amariti et al. "Holographic Optics and Negative Refractive Index". JHEP 1104 (2011), p. 036. DOI: 10.1007/JHEP04(2011)036. arXiv: 1006.5714 [hep-th].

[12] A. Amariti et al. "Negative Refraction and Superconductivity". JHEP 10 (2011), p. 104. DOI: 10.1007/JHEP10 (2011) 104. arXiv: 1107.1242 [hep-th].

[13] T. Banks, J.-F. Fortin, and S. Thomas. "Direct Detection of Dark Matter Electromagnetic Dipole Moments" (2010). arXiv: 1007.5515 [hep-ph].

[14] C. Bignamini et al. "Prompt production of loosely bound mesonic molecules at hadron colliders". PoS DIS2010 (2010), p. 176. 
[15] C. Bignamini et al. "Is the X(3872) molecular hypothesis compatible with CDF data?" PoS EPS-HEP2009 (2009), p. 074.

[16] C. Bignamini et al. "Is the X(3872) Production Cross Section at Tevatron Compatible with a Hadron Molecule Interpretation?" Phys.Rev.Lett. 103 (2009), p. 162001. DOI: 10.1103/PhysRevLett.103.162001. arXiv: 0906.0882 [hep-ph].

[17] C. Bignamini et al. "More loosely bound hadron molecules at CDF?" Phys.Lett. B684 (2010), pp. 228-230. DOI: 10.1016/j . physletb.2010.01.037. arXiv: 0912.5064 [hep-ph].

[18] F. Brazzi et al. "Strong Couplings of $X(3872)_{J=1,2}$ and a New Look at $J / \psi$ Suppression in Heavy Ion Collisions". Phys.Rev. D84 (2011), p. 014003. DOI: 10.1103/ PhysRevD.84.014003. arXiv: 1103.3155 [hep-ph].

[19] A. M. Cieplak and K. Griest. "Improved Theoretical Predictions of Microlensing Rates for the Detection of Primordial Black Hole Dark Matter". Astrophys.J. 767 (2013), p. 145 . DOI: $10.1088 / 0004-637 X / 767 / 2 / 145$. arXiv: 1210.7729 [astro-ph.CO].

[20] C. Csaki et al. "Flavor Alignment via Shining in RS". Phys.Rev. D81 (2010), p. 075025. DOI: 10.1103/PhysRevD.81.075025. arXiv: 0907.0474 [hep-ph].

[21] G. D'Amico et al. "Quasi-Dilaton: Theory and Cosmology" (2012). arXiv: 1206.4253 [hep-th].

[22] G. D'Amico et al. "On Cosmological Perturbations of Quasidilaton" (2013). arXiv: 1304.0723 [hep-th].

[23] J. Espinosa and B. Grinstein. "Ultraviolet Properties of the Higgs Sector in the LeeWick Standard Model". To appear in Physical Review D (2011). arXiv: 1101.5538 [hep-ph].

[24] Z. Fodor et al. "Calculating the running coupling in strong electroweak models". PoS LAT2009 (2009), p. 058. arXiv: 0911.2934 [hep-lat].

[25] Z. Fodor et al. "Chiral properties of SU(3) sextet fermions". JHEP 0911 (2009), p. 103. DOI: $10.1088 / 1126-6708 / 2009 / 11 / 103$. arXiv: 0908.2466 [hep-lat].

[26] Z. Fodor et al. "Chiral symmetry breaking in nearly conformal gauge theories". PoS LAT2009 (2009), p. 055. arXiv: 0911.2463 [hep-lat].

[27] Z. Fodor et al. "Nearly conformal gauge theories in finite volume". Phys.Lett. B681 (2009), pp. 353-361. DOI: 10.1016/j . physletb.2009.10.040. arXiv: 0907.4562 [hep-lat].

[28] Z. Fodor et al. "Topology and higher dimensional representations". JHEP 0908 (2009), p. 084. DOI: $10.1088 / 1126-6708 / 2009 / 08 / 084$. arXiv: 0905.3586 [hep-lat].

[29] Z. Fodor et al. "Nearly conformal gauge theories on the lattice". Int.J.Mod.Phys. A25 (2010), pp. 5162-5174. DOI: 10.1142/S0217751X10050937.

[30] Z. Fodor et al. "Chiral symmetry breaking in fundamental and sextet fermion representations of SU(3) color" (2011). arXiv: 1103.5998 [hep-lat]. 
[31] Z. Fodor et al. "Twelve massless flavors and three colors below the conformal window". Phys.Lett. B703 (2011), pp. 348-358. DOI: 10.1016/j · physletb. 2011.07 .037$. arXiv: 1104.3124 [hep-lat].

[32] Z. Fodor et al. "Can the nearly conformal sextet gauge model hide the Higgs impostor?" Phys.Lett. B718 (2012), pp. 657-666. DOI: 10.1016/j . physletb.2012.10.079. arXiv: 1209.0391 [hep-lat].

[33] Z. Fodor et al. "Confining force and running coupling with twelve fundamental and two sextet fermions". PoS LATTICE2012 (2012), p. 025. arXiv: 1211.3548 [hep-lat].

[34] Z. Fodor et al. "Lattice methods in gauge theories beyond the standard model". Prog.Theor.Phys.Suppl. 197 (2012), pp. 31-45. DOI: 10.1143/PTPS.197.31.

[35] Z. Fodor et al. "The gradient flow running coupling scheme". PoS LATTICE2012 (2012), p. 050. arXiv: 1211.3247 [hep-lat].

[36] Z. Fodor et al. "The sextet gauge model, light Higgs, and the dilaton". PoS LATTICE2012 (2012), p. 024. arXiv: 1211.6164 [hep-lat].

[37] Z. Fodor et al. "The Yang-Mills gradient flow in finite volume". JHEP 1211 (2012), p. 007. DOI: 10.1007/JHEP11(2012) 007. arXiv: 1208.1051 [hep-lat].

[38] Z. Fodor et al. "Twelve fundamental and two sextet fermion flavors" (2012). arXiv: 1205.1878 [hep-lat].

[39] B. Fornal, B. Grinstein, and M. B. Wise. "Lee-Wick Theories at High Temperature". Phys. Lett. B674 (2009), pp. 330-335. DOI: 10.1016/j . physletb.2009.03.036. arXiv: 0902.1585 [hep-th].

[40] J.-F. Fortin, B. Grinstein, and A. Stergiou. "Scale without Conformal Invariance: An Example". Phys. Lett. B704 (2011), pp. 74-80. DOI: 10.1016/j. physletb.2011.08. 060. arXiv: 1106.2540 [hep-th].

[41] J.-F. Fortin, B. Grinstein, and A. Stergiou. "Cyclic unparticle physics". Phys. Lett. B709 (2012), pp. 408-412. DOI: 10.1016/j . physletb.2012.02.046. arXiv: 1110 . 1634 [hep-th].

[42] J.-F. Fortin, B. Grinstein, and A. Stergiou. "Scale without Conformal Invariance at Three Loops" (2012). arXiv: 1202.4757 [hep-th].

[43] J.-F. Fortin, B. Grinstein, and A. Stergiou. "Scale without Conformal Invariance in Four Dimensions" (2012). arXiv: 1206.2921 [hep-th].

[44] J.-F. Fortin, B. Grinstein, and A. Stergiou. "Scale without Conformal Invariance: Theoretical Foundations". JHEP 1207 (2012), p. 025. DOI: 10.1007/JHEP07 (2012) 025. arXiv: 1107.3840 [hep-th].

[45] J.-F. Fortin, B. Grinstein, and A. Stergiou. "Limit Cycles and Conformal Invariance". JHEP 1301 (2013), p. 184. DOI: 10 . 1007 / JHEP01 (2013) 184. arXiv: 1208. 3674 [hep-th].

[46] J.-F. Fortin, K. Intriligator, and A. Stergiou. "Current OPEs in Superconformal Theories". JHEP 09 (2011), p. 071. DOI: 10.1007/JHEP09(2011)071. arXiv: 1107.1721 [hep-th]. 
[47] J.-F. Fortin, K. Intriligator, and A. Stergiou. "Superconformally Covariant OPE and General Gauge Mediation". JHEP 12 (2011), p. 064. DOI: 10.1007/JHEP12(2011) 064. arXiv: 1109.4940 [hep-th].

[48] J.-F. Fortin and A. Stergiou. "Field-theoretic Methods in Strongly-Coupled Models of General Gauge Mediation". Nucl.Phys. B873 (2013), pp. 92-115. DOI: 10.1016/j . nuclphysb. 2013.04.009. arXiv: 1212.2202 [hep-th].

[49] J.-F. Fortin and T. M. Tait. "Collider Constraints on Dipole-Interacting Dark Matter". Phys.Rev. D85 (2012), p. 063506. DOI: 10.1103/PhysRevD.85.063506. arXiv: 1103.3289 [hep-ph].

[50] J.-F. Fortin et al. "On Limit Cycles in Supersymmetric Theories". Phys.Lett. B719 (2013), pp. 170-173. DOI: 10.1016/j . physletb.2012.12.059. arXiv: 1210.2718 [hep-th].

[51] G. Gabadadze, K. Hinterbichler, and D. Pirtskhalava. "Classical Duals of Derivatively Self-Coupled Theories". Phys.Rev. D85 (2012), p. 125007. DOI: 10.1103/PhysRevD. 85.125007. arXiv: 1202.6364 [hep-th].

[52] G. Gabadadze et al. "A Covariant Master Theory for Novel Galilean Invariant Models and Massive Gravity". Phys.Rev. D86 (2012), p. 124004. DOI: 10.1103/PhysRevD . 86.124004. arXiv: 1208.5773 [hep-th].

[53] G. Gabadadze et al. "On the Potential for General Relativity and its Geometry" (2013). arXiv: 1307.2245 [hep-th].

[54] B. Grinstein. "Effects of SUSY, little Higgs, etc on B decay". PoS FPCP2009 (2009), p. 045.

[55] B. Grinstein. "Heavy Flavor Theory". eConf C090726 (2009). Talk presented at the Meeting of the Dvision of Particles and Fields of the APS, Detroit, MI, July 2009. arXiv: 0910.2422 [hep-ph].

[56] B. Grinstein. "What if? On the interplay between Serendipity, Intuition and Conjecture". PoS FPCP2010 (2010), p. 060. arXiv: 1102.4011 [hep-ph].

[57] B. Grinstein. "Strong Electroweak Symmetry Breaking" (2011). Talk presented at the XXIInd Rencontres de Blois, Loire, France, 16-20 July 2010. arXiv: 1102.4009 [hep-ph].

[58] B. Grinstein, R. Kelley, and P. Uttayarat. "Hidden fine tuning in the quark sector of little higgs models". JHEP 09 (2009), p. 040. DOI: 10.1088/1126-6708/2009/09/ 040. arXiv: 0904.1622 [hep-ph].

[59] B. Grinstein, R. Kelley, and P. Uttayarat. "Hidden Fine Tuning In The Quark Sector Of Little Higgs Models" (2011). Talk presented at the 35th International Conference of High Energy Physics - ICHEP2010, July 22-28, 2010, Paris France, PoS(ICHEP 2010)392. arXiv: 1102.4010 [hep-ph] .

[60] B. Grinstein, R. Kelley, and P. Uttayarat. "One Loop Renormalization of the Littlest Higgs Model". JHEP 1102 (2011), p. 089. DOI: 10 .1007/JHEP02(2011) 089. arXiv: 1011.0682 [hep-ph]. 
[61] B. Grinstein and C. W. Murphy. "Bottom Quark Forward-Backward Asymmetry in the Standard Model and Beyond" (2013). arXiv: 1302.6995 [hep-ph].

[62] B. Grinstein, C. W. Murphy, and M. Trott. "EWPD Constraints on Flavor Symmetric Vector Fields". JHEP 11 (2011), p. 139. DOI: 10 .1007/ JHEP11(2011) 139. arXiv: 1110.5361 [hep-ph].

[63] B. Grinstein, M. Redi, and G. Villadoro. "Low Scale Flavor Gauge Symmetries". JHEP 1011 (2010), p. 067. DOI: 10.1007/JHEP11(2010) 067. arXiv: 1009 . 2049 [hep-ph].

[64] B. Grinstein and M. Trott. "An expansion for Neutrino Phenomenology" (2012). arXiv: 1203.4410 [hep-ph].

[65] B. Grinstein and P. Uttayarat. "A Very Light Dilaton". JHEP 1107 (2011), p. 038. DOI: 10.1007/JHEP07 (2011) 038. arXiv: 1105.2370 [hep-ph].

[66] B. Grinstein et al. "Flavor Symmetric Sectors and Collider Physics". JHEP 1110 (2011), p. 072. DOI: 10.1007/JHEP10 (2011) 072. arXiv: 1108.4027 [hep-ph].

[67] B. Grinstein et al. "Forward-backward asymmetry in $t \bar{t}$ production from flavour symmetries". Phys.Rev.Lett. 107 (2011), p. 012002. DOI: 10.1103/PhysRevLett . 107. 012002. arXiv: 1102.3374 [hep-ph].

[68] B. Grinstein et al. "Massive Spin-2 States as the Origin of the Top Quark ForwardBackward Asymmetry". JHEP 1208 (2012), p. 073. DOI: 10.1007/JHEP08(2012) 073. arXiv: 1203.2183 [hep-ph].

[69] M. Ibe, A. Rajaraman, and Z. Surujon. "Does Supersymmetry Require Two Higgs Doublets?" (2010). arXiv: 1012.5099 [hep-ph].

[70] K. Intriligator and N. Seiberg. "Aspects of 3d N=2 Chern-Simons-Matter Theories" (2013). arXiv: 1305.1633 [hep-th].

[71] K. Intriligator et al. "Conifold Transitions in M-theory on Calabi-Yau Fourfolds with Background Fluxes" (2012). arXiv: 1203.6662 [hep-th].

[72] N. Mekareeya, J. Song, and Y. Tachikawa. "2d TQFT structure of the superconformal indices with outer-automorphism twists". JHEP 1303 (2013), p. 171. DOI: 10.1007/ JHEP03(2013) 171. arXiv: 1212.0545 [hep-th].

[73] D. S. Park and J. Song. "The Seiberg-Witten Kahler Potential as a Two-Sphere Partition Function". JHEP 1301 (2013), p. 142. DOI: 10 . 1007/JHEP01(2013) 142. arXiv: 1211.0019 [hep-th].

[74] D. Pirtskhalava and P. Uttayarat. "CP Violation and Flavor SU(3) Breaking in Dmeson Decays" (2011). arXiv: 1112.5451 [hep-ph].

[75] C. de Rham et al. "Non-Renormalization and Naturalness in a Class of Scalar-Tensor Theories" (2012). DOI: 10.1103/PhysRevD.87.085017. arXiv: 1212.4128 [hep-th].

[76] A. Stergiou. "The chet package" (2011). arXiv: 1106.2809 [physics.gen-ph].

[77] D. C. Stone and P. Uttayarat. "Explaining the ttbar Forward-Backward Asymmetry from a GUT-Inspired Model". JHEP 1201 (2012), p. 096. DOI: 10.1007/JHEP01 (2012) 096. arXiv: 1111.2050 [hep-ph]. 
[78] Z. Surujon and P. Uttayarat. "Spontaneous CP Violation and Light Particles in The Littlest Higgs". Phys.Rev. D83 (2011), p. 076010. DOI: 10 . 1103/PhysRevD . 83. 076010. arXiv: 1003.4779 [hep-ph]. 


\section{People}

List of personnel supported by the grant. When support is for less than full time, the total number of months supported by the grant over the whole period (July 1, 2010 - April $30,2013)$ is indicated.

Faculty:

\begin{tabular}{|l|c|c|}
\hline Name & Support (months) & Other source \\
\hline Kim Griest & 6 & UCSD \\
Benjamin Grinstein & 6 & UCSD \\
Kenneth Intriligator & 6 & UCSD \\
Julius Kuti & 6 & UCSD \\
\hline
\end{tabular}

Postdocs:

\begin{tabular}{|l|c|}
\hline Name & Support \\
\hline Jean Francois Fortin & Full \\
Antonio Amariti & Full \\
Chim Kim Wong & Full \\
David Pirstkhalava & Full \\
Jaewon Song & Full \\
Ze'ev Surujon & Full \\
\hline
\end{tabular}

Students:

\begin{tabular}{|l|c|c|}
\hline Name & Support (months) & Other source \\
\hline Andreas Stergiou & 22.5 & UCSD \\
Prarit Agarwal & 11.5 & UCSD \\
David Stone & 9.5 & UCSD \\
Christopher Murphy & 8.5 & UCSD \\
Patipan Uttayarat & 11.5 & UCSD \\
Joseph Fedrow & 1 & UCSD \\
Nathan Johnson & 1 & UCSD \\
Agnieszka Cieplak & 18 & NSF \\
\hline
\end{tabular}




\title{
Task T2:
}

\section{Theoretical Particle Physics}

\author{
University of California, San Diego
}

Final Report for the period July 1, 2010 to April 30, 2013.

July 31, 2013

Elizabeth Jenkins

Aneesh Manohar ejenkins@ucsd.edu

amanohar@ucsd.edu 


\section{Contents}

Research Summary 1

Contract Publications $2010-2012 \ldots \ldots \ldots$. . . . . . . . . . 8

Invited Conference Talks . . . . . . . . . . . . . . . . . . . . . . 9 9

People . . . . . . . . . . . . . . . . . . . . . . 10 
Task T2 supports the research work of Professors Jenkins and Manohar. This report gives a detailed description of research in year 3, as well as a list of publications and conference talks during the entire 3 year period of the grant. A detailed description of the first two years research under the current grant was given in the Year 1 and Year 2 progress reports.

\section{Research Summary}

\section{Overview of Research}

The research topics studied in the last three years include neutrino physics and neutrino flavor invariants, the topology of the universe from the cosmic microwave background, electroweak radiative corrections using soft-collinear effective theory, double parton scattering, gravitational lensing, and the study of track-based observables at the LHC.

\section{Baryon Magnetic moments in the $1 / N_{c}$ Expansion}

Recent first observations of the baryon transition magnetic moments $\Sigma^{* 0} \rightarrow \Lambda$ and $\Sigma^{*+} \rightarrow$ $\Sigma^{+}$by the CLAS collaboration show that $S U(3)$ flavor symmetry breaking in the transition baryon magnetic moments is large. A complete analysis of the baryon magnetic moments to all orders in $1 / N_{c}$ and flavor symmetry breaking was performed recently in Ref. [1] by the PI. The successive sequence of fits to the baryon magnetic moments performed in this paper clearly display the hierarchy of the combined expansion. All baryon magnetic moments were predicted with a $1 \sigma$ theory error of $0.025 \mu_{N}$ by retaining the leading operators of the combined expansion. These model-independent predictions will be tested as more of the magnetic moments and transition magnetic moments are measured. The accuracy of the $1 / N_{c}$ expansion for baryon properties can be illustrated by earlier work on the baryon mass predictions shown in the table below. 


\begin{tabular}{c|ll}
\hline Theory & \multicolumn{1}{|c}{ Experiment } & Detector (Year) \\
\hline$\Xi_{c}^{\prime}=2580.8 \pm 2.1$ & $\Xi_{c}^{\prime}=2576.5 \pm 2.3$ & CLEO(1999) \\
$\Omega_{c}^{*}=2760.5 \pm 4.9$ & $\Omega_{c}^{*}=2768.3 \pm 3.0$ & BaBar (2006) \\
$\Xi_{b}=5805.7 \pm 8.1$ & $\Xi_{b}^{-}=5774 \pm 11 \pm 15$ & D0 (2007) \\
& $\Xi_{b}^{-}=5792.9 \pm 2.5 \pm 1.7$ & CDF (2007) \\
$\Sigma_{b}=5824.2 \pm 9.0$ & $\Sigma_{b}=5811.5 \pm 1.7$ & CDF (2007) \\
$\Sigma_{b}^{*}=5840.0 \pm 8.8$ & $\Sigma_{b}^{*}=5832.7 \pm 1.8$ & CDF (2007) \\
$\Omega_{b}=6039.1 \pm 8.3$ & $\Omega_{b}=6054.4 \pm 6.8 \pm 0.9$ & CDF (2009) \\
$\Xi_{b}^{*}=5950.3 \pm 4.2$ & $\Xi_{b}^{* 0}=5945.0 \pm 0.7 \pm 0.3 \pm 2.7$ & CMS (2012)
\end{tabular}

Comparison of the $1 / N_{c}$ mass predictions with experiment. All masses are in MeV. For all of the listed baryon masses, the theoretical prediction was made before the experimental measurement.

\section{Double Parton Scattering}

In high-energy hadronic collisions, one parton from each hadron can collide via a hard interaction to produce a final state with a large invariant mass. The classic example of such single parton scattering is Drell-Yan production, $p_{1}+p_{2} \rightarrow \ell^{+} \ell^{-}$. In some hadronic collisions, two partons in one hadron can have simultaneous hard interactions with two partons from the other hadron. This process is called double parton scattering (DPS).

A systematic QCD analysis of double parton scattering was done in Refs. [2, 3] in collaboration with Waalewijn. The cross-section was determined using a QCD factorization theorem in terms of double parton distribution functions (dPDFs) and soft functions. The one-loop QCD evolution of the dPDFs was also worked out. Previous attempts to do a complete one-loop analysis of double parton scattering were stymied by rapidity divergences. We were able to solve this problem using the CMU rapidity regulator.

We were also able to solve a long-standing problem in double parton scattering. The double parton distribution mixes under QCD evolution with the gluon single PDF

$$
\begin{aligned}
\mu \frac{\mathrm{d} F_{q \bar{q}}^{1}\left(x_{1}, x_{2}, \mathbf{z}_{\perp}\right)}{\mathrm{d} \mu} & =\frac{\alpha_{s}}{\pi} \delta^{(2)}\left(\mathbf{z}_{\perp}\right) P_{q g}\left(\frac{x_{1}}{x_{1}+x_{2}}\right) \frac{f_{g}\left(x_{1}+x_{2}\right)}{x_{1}+x_{2}}+\ldots \\
P_{q g}(x) & =T_{F}\left[x^{2}+(1-x)^{2}\right]
\end{aligned}
$$

where $F_{q \bar{q}}^{1}\left(x_{1}, x_{2}, \mathbf{z}_{\perp}\right)$ is the $\mathrm{dPDF}$, and is the probability to find a $q$ and $\bar{q}$ with momentum fractions $x_{1,2}$ and separated by $\mathbf{z}_{\perp} . P_{q g}(x)$ is the usual splitting function. The "..." denote non-mixing contributions and are not important for the present discussion. The $\delta^{(2)}\left(\mathbf{z}_{\perp}\right)$ arises because the gluon splits into a $q \bar{q}$ pair at the same point. The $\delta^{(2)}\left(\mathbf{z}_{\perp}\right)$ is problematic and leads to infinities in the cross section from the convolution of two dPDFs. In Ref. [2] we showed how this problem is solved by a careful treatment of the renormalization of composite operators. As a result, DPS is now on the same solid theoretical foundation as single parton scattering processes such as Drell-Yan, and experimental data can be used to extract the double parton distribution functions. 

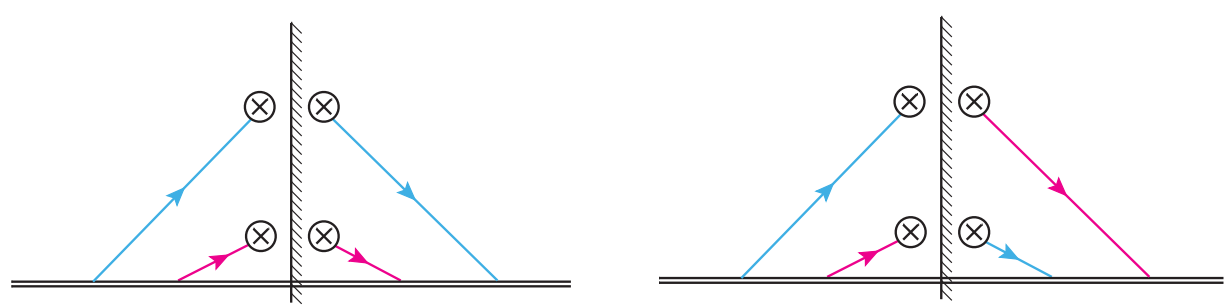

Figure 1: Schematic representation of the $q q$ double parton distribution. The two $\otimes$ are separated vertically by $\mathbf{z}_{\perp}$. One can have color-direct and color-exchange dPDFs.

The double parton distribution functions are represented graphically in Fig. 1. They represent the probability to extract two partons from a hadron. There are non-trivial spin and color correlations that are measured by the double parton distributions as can be seen from the figure. The two quarks can be extracted from the hadron and then put back either directly, or with their color exchanged, and similarly for the spin. This does not affect the spin or color of the hadron target.

The quark spin in a proton is correlated with the hadron spin, so that both $\Delta q(x)$, the longitudinally polarized parton distribution, and $\delta q(x)$, the transversely polarized parton distribution, vanish for unpolarized proton targets. By contrast, in double parton scattering the spins of the two partons can be correlated with each other, and so nontrivial spin structures exist even if the proton is unpolarized. In addition to the usual unpolarized distribution $F_{q q}$, there are two other distributions $F_{\Delta q \Delta q}$, and $F_{\delta q \delta q}$, which measure longitudinal and transverse spin correlations between two partons. There are also non-trivial flavor correlations. For example, in a naive quark model, the $d d \mathrm{dPDF}$ in the proton should vanish, since the proton has quark content $u u d$, whereas the $u u$ dPDF should be non-zero.

We obtained a first estimate of the size of these correlations by calculating the corresponding double parton distribution functions in a bag model of the proton. We find significant correlations between momentum fractions, spin and flavor, but negligible correlations with transverse separation. The model estimates of the relative importance of these correlations will help experimental studies disentangle them.

\section{Fragmentation with a Cut on Thrust: Predictions for B-factories.}

When high-energy single-hadron production takes place inside an identified jet, there are important correlations between the fragmentation and phase-space cuts. For example, when one-hadron yields from light-quark fragmentation are measured in on-resonance B-factory data, a cut on the thrust event shape $T$ is required to remove the large b-quark contribution, leading to a dijet final state restriction. Here we complete our analysis of unpolarized fragmentation of (light) quarks and gluons to a light hadron $h$ with energy fraction $z$ in $e^{+} e^{-} \rightarrow$ dijet $+h$ at the center-of-mass energy $Q=10.58 \mathrm{GeV}$. In addition to the next-tonext-to-leading order resummation of logarithms of $1-T$, we include the next-to-leading order nonsingular $\mathcal{O}(1-T)$ contribution to the cross section, the resummation of threshold logarithms of $1-z$, and the leading nonperturbative contribution to the soft function. Our 
results for the correlations between fragmentation and the thrust cut are presented in a way that can be directly tested against B-factory data. These correlations are also observed in Pythia.

\section{Jet Charge at the LHC.}

Knowing the charge of the parton initiating a light-quark jet could be extremely useful both for testing aspects of the Standard Model and for characterizing potential beyondthe-Standard-Model signals. We show that despite the complications of hadronization and out-of-jet radiation such as pile-up, a weighted sum of the charges of a jet's constituents can be used at the LHC to distinguish among jets with different charges. Potential applications include measuring electroweak quantum numbers of hadronically decaying resonances or supersymmetric particles, as well as Standard Model tests, such as jet charge in dijet events or in hadronically-decaying $\mathrm{W}$ bosons in t-tbar events. We develop a systematically improvable method to calculate the average and width of the jet charge distributions by combining (multi-hadron) fragmentation functions with perturbative jet functions and perturbative evolution equations. We show that the dependence on energy and jet size for the average and width of the jet charge can be calculated despite the large experimental uncertainty on fragmentation functions, providing a validation tool for data independent of Monte-Carlo fragmentation models.

\section{Calculating the Charge of a Jet.}

Jet charge has played an important role in experimentally testing the Parton Model and the Standard Model, and has many potential LHC applications. The energy-weighted charge of a jet is not an infrared-safe quantity, so hadronization must be taken into account. Here we go beyond the average and width of the jet charge distribution and develop the formalism to calculate the full jet charge distribution. We cleanly separate the nonperturbative from the perturbative physics, which we compute at one-loop order. We find that the renormalization group evolution of the nonperturbative ingredients has a natural parton shower implementation. Our numerical results are compared to Pythia and agree well in almost all cases.

\section{Combining Fixed-Order Helicity Amplitudes With Resummation Using SCET.}

We discuss how to construct a simple and easy-to-use helicity operator basis in SoftCollinear Effective Theory (SCET), for which the hard Wilson coefficients from matching QCD onto SCET are directly given in terms of the color-ordered QCD helicity amplitudes. This provides an interface to seamlessly combine fixed-order helicity amplitudes, which are the basic building blocks of state-of-the-art next-to-leading order calculations for multileg processes, with a resummation of higher-order logarithmic corrections using SCET. 


\section{Measuring gravitational lensing of the cosmic microwave background using cross correlation with large scale structure.}

We cross correlate the gravitational lensing map extracted from cosmic microwave background measurements by the Wilkinson Microwave Anisotropy Probe (WMAP) with the radio galaxy distribution from the NRAO VLA Sky Survey (NVSS) by using a quadratic estimator technique. We use the full covariance matrix to filter the data, and calculate the cross-power spectra for the lensing-galaxy correlation. We explore the impact of changing the values of cosmological parameters on the lensing reconstruction, and obtain statistical detection significances at $>3 \sigma$. The results of all cross correlations pass the curl null test as well as a complementary diagnostic test using the NVSS data in equatorial coordinates. We forecast the potential for Planck and NVSS to constrain the lensing-galaxy cross correlation as well as the galaxy bias. The lensing-galaxy cross-power spectra are found to be Gaussian distributed.

\section{Jet Mass Spectra in Higgs + One Jet at NNLL.}

The invariant mass of a jet is a benchmark variable describing the structure of jets at the LHC. We calculate the jet mass spectrum for Higgs plus one jet at the LHC at next-to-nextto-leading logarithmic (NNLL) order using a factorization formula. At this order, the cross

section becomes sensitive to perturbation theory at the soft $m_{\text {jet }}^{2} / p_{T}^{\text {jet }}$ scale. Our calculation is exclusive, i.e. it involves a veto on additional jets. However, the dominant effect of this jet veto is removed by normalizing the spectrum. We present results for the dependence of the jet mass spectrum on the kinematics, jet algorithm, and jet size $R$. Using individual partonic channels we illustrate the difference between the jet mass spectra for quark and gluon jets. We also study the effect of hadronization and underlying event on the jet mass in PYTHIA. To highlight the similarity of inclusive and exclusive jet mass spectra, a comparison to LHC data is presented.

\section{Calculating Track-Based Observables for the LHC.}

By using observables that only depend on charged particles (tracks), one can efficiently suppress pile-up contamination at the LHC. Such measurements are not infrared safe in perturbation theory, so any calculation of track-based observables must account for hadronization effects. We develop a formalism to perform these calculations in QCD, by matching partonic cross sections onto new non-perturbative objects called track functions which absorb infrared divergences. The track function $T_{i}(x)$ describes the energy fraction $x$ of a hard parton $i$ which is converted into charged hadrons. We give a field-theoretic definition of the track function and derive its renormalization group evolution, which is in excellent agreement with the Pythia parton shower. We then perform a next-to-leading order calculation of the total energy fraction of charged particles in $e^{+} e^{-} \rightarrow$ hadrons. To demonstrate the implications of our framework for the LHC, we match the Pythia parton shower onto a set of track functions to describe the track mass distribution in Higgs plus one jet events. We also show how to reduce smearing due to hadronization fluctuations by measuring dimensionless track-based ratios. 


\section{Calculating Track Thrust with Track Functions.}

In $e^{+} e^{-}$event shapes studies at LEP, two different measurements were sometimes performed: a "calorimetric" measurement using both charged and neutral particles, and a "track-based" measurement using just charged particles. Whereas calorimetric measurements are infrared and collinear safe and therefore calculable in perturbative QCD, trackbased measurements necessarily depend on non-perturbative hadronization effects. On the other hand, track-based measurements typically have smaller experimental uncertainties. We present the first calculation of the event shape track thrust and compare to measurements performed at ALEPH and DELPHI. This calculation is made possible through our track function formalism, and is carried out at next-to-leading logarithmic order. Due to a partial cancellation between non-perturbative parameters, the distributions for calorimeter thrust and track thrust are remarkably similar, a feature also seen in LEP data.

\section{Review of Particle Physics}

A. Manohar

Manohar is a member of the Particle Data Group and contributes to the Review of Particle Physics. 


\section{Contract Publications 2010-2012}

[1] H.-M. Chang et al. "Double Parton Correlations in the Bag Model". Phys.Rev. D87 (2013), p. 034009. DOI: 10.1103/PhysRevD.87.034009. arXiv:1211.3132 [hep-ph].

[2] I. W. Stewart et al. "Combining Fixed-Order Helicity Amplitudes With Resummation Using SCET". PoS LL2012 (2012), p. 058. arXiv:1211.2305 [hep-ph].

[3] W. J. Waalewijn. "Calculating the Charge of a Jet". Phys.Rev. D86 (2012), p. 094030. DOI: 10.1103/PhysRevD.86.094030. arXiv:1209.3019 [hep-ph].

[4] D. Krohn et al. "Jet Charge at the LHC". Phys. Rev. Lett. 110 (2013), p. 212001. DOI: 10.1103/PhysRevLett.110.212001. arXiv:1209.2421 [hep-ph].

[5] J. Beringer et al. "Review of Particle Physics (RPP)". Phys.Rev. D86 (2012), p. 010001. DOI: 10.1103/PhysRevD.86.010001.

[6] A. Jain et al. "Fragmentation with a Cut on Thrust: Predictions for B-factories" (2012). DOI: 10.1103/PhysRevD.87.074013. arXiv:1207.4788 [hep-ph].

[7] C. Feng et al. "Measuring Gravitational Lensing of the Cosmic Microwave Background using cross-correlation with large scale structure". Phys.Rev. D86 (2012), p. 063519. DOI: 10.1103/PhysRevD.86.063519. arXiv:1207.3326 [astro-ph.CO].

[8] A. V. Manohar and W. J. Waalewijn. "What is Double Parton Scattering?" Phys.Lett. B713 (2012), pp. 196-201. DOI: 10.1016/j . physletb. 2012.05.044. arXiv:1202. 5034 [hep-ph].

[9] A. V. Manohar and W. J. Waalewijn. "A QCD Analysis of Double Parton Scattering: Color Correlations, Interference Effects and Evolution". Phys.Rev. D85 (2012), p. 114009. DOI: 10.1103/PhysRevD.85.114009. arXiv:1202.3794 [hep-ph].

[10] A. V. Manohar and M. Trott. "Electroweak Sudakov Corrections and the Top Quark Forward-Backward Asymmetry". Phys.Lett. B711 (2012), pp. 313-316. DOI: 10.1016/ j.physletb. 2012.04.013. arXiv:1201.3926 [hep-ph].

[11] S. Dittmaier et al. "Handbook of LHC Higgs Cross Sections: 2. Differential Distributions" (2012). arXiv:1201.3084 [hep-ph].

[12] M. Procura and W. J. Waalewijn. "Fragmentation in Jets: Cone and Threshold Effects". Phys.Rev. D85 (2012), p. 114041. DOI: 10.1103/PhysRevD . 85.114041. arXiv:1111. 6605 [hep-ph].

[13] E. E. Jenkins. "Baryon Magnetic Moments in the $1 / N_{c}$ Expansion with Flavor Symmetry Breaking". Phys.Rev. D85 (2012), p. 065007. DOI: 10.1103/PhysRevD.85.065007. arXiv:1111.2055 [hep-ph].

[14] A. Jain et al. "Fully-Unintegrated Parton Distribution and Fragmentation Functions at Perturbative $k_{T}$ ". JHEP 1204 (2012), p. 132. DOI: 10 . 1007/ JHEP04(2012) 132. arXiv:1110.0839 [hep-ph].

[15] W. J. Waalewijn. "Factorization at the LHC: From PDFs to Initial State Jets" (2010). arXiv:1104.5164 [hep-ph]. 
[16] G. Aslanyan and A. V. Manohar. "The Topology and Size of the Universe from the Cosmic Microwave Background". JCAP 1206 (2012), p. 003. DOI: 10 . 1088/14757516/2012/06/003. arXiv:1104.0015 [astro-ph.CO].

[17] T. T. Jouttenus et al. "The Soft Function for Exclusive N-Jet Production at Hadron Colliders". Phys.Rev. D83 (2011), p. 114030. DOI: 10 . 1103/PhysRevD . 83 . 114030. arXiv:1102.4344 [hep-ph].

[18] A. Jain et al. "Parton Fragmentation within an Identified Jet at NNLL". JHEP 1105 (2011), p. 035. DOI: 10.1007/JHEP05(2011)035. arXiv:1101.4953 [hep-ph].

[19] C. F. Berger et al. "Higgs Production with a Central Jet Veto at NNLL+NNLO". JHEP 1104 (2011), p. 092. DOI: 10.1007/JHEP04 (2011) 092. arXiv:1012.4480 [hep-ph].

[20] K. Nakamura et al. "Review of particle physics". J.Phys. G37 (2010), p. 075021. DOI: 10.1088/0954-3899/37/7A/075021.

[21] A. Fuhrer et al. "Electroweak radiative Corrections to Higgs Production via Vector Boson Fusion using Soft-Collinear Effective Theory". Phys.Rev. D84 (2011), p. 013007. DOI: 10.1103/PhysRevD.84.013007. arXiv:1011.1505 [hep-ph].

[22] A. Hanany et al. "Hilbert Series for Flavor Invariants of the Standard Model". JHEP 1103 (2011), p. 096. DOI: 10.1007/JHEP03 (2011)096. arXiv:1010.3161 [hep-ph].

[23] A. Fuhrer. "Pion electroproduction in a nonrelativistic theory". Phys.Lett. B692 (2010), pp. 130-135. DOI: 10.1016/j.physletb.2010.07.029. arXiv:1007.0031 [hep-ph].

[24] A. V. Manohar. "Reparametrization Invariance Constraints on Inclusive Decay Spectra and Masses". Phys.Rev. D82 (2010), p. 014009. DOI: 10.1103/PhysRevD.82.014009. arXiv:1005.1952 [hep-ph]. 


\section{Invited Conference Talks 2010-2012}

[1] A. V. Manohar. Double Parton Scattering. ConfX, Munich, Germany, Oct. 2012.

[2] E. E. Jenkins. Neutrino Flavor Invariants. invited talk at the neutrino workshop, Galileo Galilei Institute. Florence, Italy, July 2012.

[3] A. V. Manohar. Electroweak Corrections at the LHC. GGI, Florence, Italy, July 2012.

[4] A. V. Manohar. The Higgs Boson. invited public lecture at the National Center for Biological Studies. Bangalore, India, July 2012.

[5] W. J. Waalewijn. Correlations in Double Parton Scattering. Pheno 2012. May 2012.

[6] W. J. Waalewijn. The Charge of a Jet. Invited Talk, LoopFest XI. Pittsburgh, May 2012.

[7] A. V. Manohar. Double Parton Scattering. invited talk, SCET 2012. Madrid, Spain, Mar. 2012.

[8] W. J. Waalewijn. Employing Helicity Amplitudes for Resummation in SCET. INT Workshop "Frontiers in QCD", University of Washington. Seattle, Washington, Sept. 2011.

[9] W. J. Waalewijn. Higgs Production with a Jet Veto at NNLL+NNLO. invited talk, SCET 2011. Carnegie Mellon University, Mar. 2011.

[10] W. J. Waalewijn. Higgs Production with a Jet Veto at NNLL+NNLO. Invited talk, West Coast LHC Theory Meeting, UC Irvine. Seattle, Washington, Dec. 2010.

[11] A. V. Manohar. 1/N and Baryons. invited talk, QCD and Strings: Elements of a Universal Theory. Oberwölz, Austria, Sept. 2010.

[12] A. V. Manohar. Factorization Structure of Gauge Theory Amplitudes and Application to High Energy Scattering at the LHC. invited plenary talk, Quark Confinement and Hadron Spectroscopy. Madrid, Spain, Aug. 2010.

[13] W. J. Waalewijn. Higgs Production with a Jet Veto at $N N L L+N N L O$. Invited talk, Boston Jet Physics Workshop. MIT, Jan. 2010. 
List of personnel supported by the grant.

Faculty:

\begin{tabular}{|c|c|c|}
\hline Name & Support & Other Source \\
\hline Elizabeth Jenkins & 6 months $/ 3$ years & UCSD \\
Aneesh Manohar & 6 months $/ 3$ years & UCSD \\
\hline
\end{tabular}

Postdocs:

\begin{tabular}{|c|c|}
\hline Name & Support \\
\hline Wouter Waalewijn & Full \\
\hline
\end{tabular}

Students:

\begin{tabular}{|c|c|c|}
\hline Name & Support & Other Source \\
\hline Grigor Aslanyan & partial & UCSD \\
Hsi-Ming Chang & partial & UCSD \\
Brian Shotwell & partial & UCSD \\
\hline
\end{tabular}

